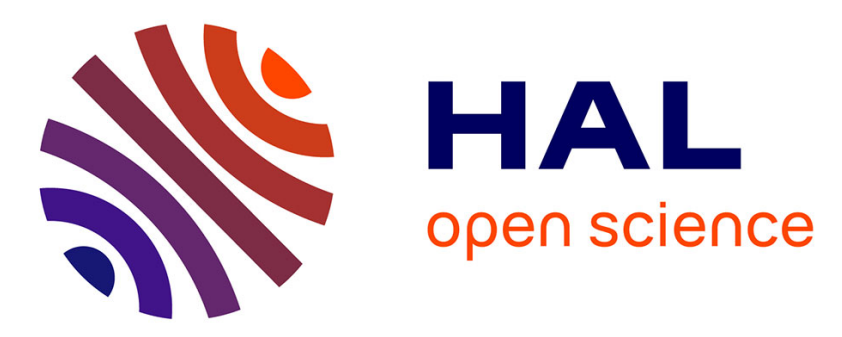

\title{
Random vectors and random fields in high dimension. Parametric model-based representation, identification from data, and inverse problems \\ Christian Soize
}

\section{- To cite this version:}

Christian Soize. Random vectors and random fields in high dimension. Parametric model-based representation, identification from data, and inverse problems. R. Ghanem, D. Higdon, and H. Owhadi. Handbook for Uncertainty Quantification, 2, Springer International Publishing Switzerland, pp.883935, 2017, 978-3-319-12384-4. 10.1007/978-3-319-11259-6

${ }_{3} 0-1$. hal -01284672

\section{HAL Id: hal-01284672 \\ https://hal.science/hal-01284672}

Submitted on 7 Mar 2016

HAL is a multi-disciplinary open access archive for the deposit and dissemination of scientific research documents, whether they are published or not. The documents may come from teaching and research institutions in France or abroad, or from public or private research centers.
L'archive ouverte pluridisciplinaire HAL, est destinée au dépôt et à la diffusion de documents scientifiques de niveau recherche, publiés ou non, émanant des établissements d'enseignement et de recherche français ou étrangers, des laboratoires publics ou privés. 


\title{
Random vectors and random fields in high dimension. Parametric model-based representation, identification from data, and inverse problems
}

\author{
C. Soize ${ }^{1}$ \\ Université Paris-Est, Laboratoire Modélisation et Simulation Multi-Echelle, MSME UMR 8208 \\ CNRS, 5 bd Descartes, 77454 Marne-la-Vallée, France
}

\begin{abstract}
The statistical inverse problem for the experimental identification of a non-Gaussian matrix-valued random field that is the model parameter of a boundary value problem, using some partial and limited experimental data related to a model observation, is a very difficult and challenging problem. A complete advanced methodology and the associated tools are presented for solving such a problem in the following framework: the random field that must be identified is a nonGaussian matrix-valued random field and is not simply a real-valued random field; this non-Gaussian random field is in high stochastic dimension and is identified in a general class of random fields; some fundamental algebraic properties of this non-Gaussian random field must be satisfied such as symmetry, positiveness, invertibility in mean square, boundedness, symmetry class, spatial-correlation lengths, etc; the available experimental data sets correspond only to partial and limited data for a model observation of the boundary value problem.

The developments presented are mainly related to the elasticity framework, but the methodology is general and can be used in many areas of computational sciences and engineering. The developments are organized as follows. The first part is devoted to the definition of the statistical inverse problem that has to be solved in high stochastic dimension, and is focussed on stochastic elliptic operators such that the ones that are encountered in the boundary value problems of the linear elasticity. The second one deals with the construction of two possible parameterized representations for a non-Gaussian positive-definite matrix-valued random field that models the model parameter of a boundary value problem. A
\end{abstract}

\footnotetext{
${ }^{1}$ Corresponding author, christian.soize@univ-paris-est.fr
} 
parametric model-based representation is then constructed in introducing a statistical reduced model and a polynomial chaos expansion, first with deterministic coefficients and after with random coefficients. This parametric model-based representation is directly used for solving the statistical inverse problem. The third part is devoted to the description of all the steps of the methodology allowing the statistical inverse problem to be solved in high stochastic dimension. These steps are based on the identification of a prior stochastic model of the Non-Gaussian random field by using the maximum likelihood method and then, on the identification of a posterior stochastic model of the Non-Gaussian random field by using the Bayes method.The fourth part presents the construction of an algebraic prior stochastic model of the model parameter of the boundary value problem, for a non-Gaussian matrix-valued random field. The generator of realizations for such an algebraic prior stochastic model for a non-Gaussian matrix-valued random field is presented.

Key words: Random vector, Random field, Random Matrix, High dimension, High stochastic dimension, Non-Gaussian, Non-gaussian random field, Representation of random fields, Polynomial chaos expansion, Generator, Maximum entropy principle, Prior model, Maximum likelihood method, Bayesian method, Identification, Inverse problem, Statistical inverse problem, Random media, Heterogeneous microstructure, Composite materials, Porous media.

\section{Introduction}

The statistical inverse problem for the experimental identification of a nonGaussian matrix-valued random field that is the model parameter of a boundary value problem, using some partial and limited experimental data related to a model observation, is a very difficult and challenging problem. The classical methodologies that are very efficient for Gaussian random fields, cannot be used for nonGaussian matrix-valued random fields in high stochastic dimension, in particular under the assumption that only partial and limited experimental data are available for the statistical inverse problem that has to be solved for identifying the nonGaussian random field through a boundary value problem. This means that experimental data must be enriched in introducing adapted informative prior stochastic models for the non-Gaussian matrix-valued random fields in order to take into account fundamental algebraic properties such as symmetry, positiveness, invertibility in mean square, boundedness, symmetry class, spatial-correlation lengths, 
etc. The objective is then to present a complete advanced methodology and the associated tools for solving such a statistical inverse problem in high stochastic dimension and related to non-Gaussian matrix-valued random fields.

\section{Notions on the High Stochastic Dimension and on the Parametric Model- Based Representations for Random Fields}

What is a random vector or a random field with a high stochastic dimension? The stochastic dimension of a random vector or a random field is an important notion that allows for evaluating the level of complexity of a statistical inverse problem related to the identification of a random model parameter (random vector, random field) of a stochastic boundary value problem (for instance, the coefficients of a partial differential equation) using experimental data related to a random model observation (random variable, random vector, random field) of this boundary value problem.

Let us consider a random vector $\mathbf{U}$ with values in $\mathbb{R}^{N_{U}}$ in which $N_{U}$ is an integer. The stochastic dimension of $\mathbf{U}$ is not, in general, the value of integer $N_{U}$. For instance, if $\mathbf{U}$ is written as $\mathbf{U}=\eta \mathbf{b}$, in which $\eta$ is a real-valued random variable and where $\mathbf{b}$ is a deterministic vector given in $\mathbb{R}^{N_{U}}$, then the stochastic dimension of $\mathbf{U}$ is 1 for any value of integer $N_{U}$. If $\mathbf{U}$ is written as $\mathbf{U}=\sum_{i=1}^{m} \eta_{i} \mathbf{b}^{i}$ with $m \leq N_{U}$, in which $\eta_{1}, \ldots, \eta_{m}$ are $m$ independent real-valued random variables and where $\mathbf{b}^{1}, \ldots, \mathbf{b}^{m}$ are $m$ algebraically independent vectors given in $\mathbb{R}^{N_{U}}$, then the stochastic dimension of $\mathbf{U}$ is $m$, and $\mathbf{U}$ is in high stochastic dimension if $m$ is large. If $\mathbf{U}$ is a second-order random vector whose covariance matrix is known, then the use of the principal component analysis allows the reduced representation $\mathbf{U}^{(m)}=\sum_{i=1}^{m} \eta_{i} \sqrt{\lambda_{i}} \mathbf{b}^{i}$ of $\mathbf{U}$ to be constructed with $m<N_{U}$, and where $m$ is calculated in order that the mean-square error of $\mathbf{U}-\mathbf{U}^{(m)}$ is sufficiently small. It can thus be written $\mathbf{U} \sim \mathbf{U}^{(m)}$ (in mean square). In such a reduced representation, $\lambda_{1} \geq \ldots>\lambda_{m}>0$ are the dominant eigenvalues of the covariance matrix of $\mathbf{U}$ and $\mathbf{b}^{1}, \ldots, \mathbf{b}^{m}$ are the associated orthonormal eigenvectors in $\mathbb{R}^{N_{U}}$. The components $\eta_{1}, \ldots, \eta_{m}$ are $m$ centered and uncorrelated real-valued random variables. If random vector $\mathbf{U}$ is a Gaussian random vector, then $\eta_{1}, \ldots, \eta_{m}$ are $m$ independent Gaussian real-valued random variables, and, for this particular Gaussian case, the stochastic dimension of $\mathbf{U}$ is $m$. However, for the general case, $\mathbf{U}$ is a non-Gaussian random vector, and consequently, the real-valued random variables $\eta_{1}, \ldots, \eta_{m}$ (that are centered and uncorrelated) are not independent but are statistically dependent. In such a case, $m$ is not the stochastic dimension of $\mathbf{U}$, but clearly the stochastic dimension is less or equal to $m$ (the equality is obtained for 
the Gaussian case). Let us assume that there exists a deterministic nonlinear mapping $\mathcal{Y}$ from $\mathbb{R}^{N_{g}}$ into $\mathbb{R}^{m}$ such that the random vector $\boldsymbol{\eta}=\left(\eta_{1}, \ldots, \eta_{m}\right)$ can be written as $\boldsymbol{\eta}=\mathcal{Y}\left(\Xi_{1}, \ldots, \Xi_{N_{g}}\right)$ in which $N_{g}<m$ and where $\Xi_{1}, \ldots, \Xi_{N_{g}}$ are $N_{g}$ independent real-valued random variables (for instance, $\mathcal{Y}$ can be constructed using the polynomial chaos expansion of the second-order random vector $\boldsymbol{\eta}$ ). In such a case, the stochastic dimension of $\mathbf{U}$ is less or equal to $N_{g}$. If among all the possible nonlinear mappings and all the possible integers $N_{g}$ such that $1 \leq N_{g}<m$, the mapping $\mathcal{Y}$ and the integer $N_{g}$ correspond to the smallest possible value of $N_{g}$ such as $\boldsymbol{\eta}=\mathcal{Y}\left(\Xi_{1}, \ldots, \Xi_{N_{g}}\right)$, then $N_{g}$ is the stochastic dimension of $\mathbf{U}$, and $\mathbf{U}$ has a high stochastic dimension if $N_{g}$ is large.

If $\{\mathbf{u}(\mathbf{x}), \mathbf{x} \in \Omega\}$ is a second-order random field indexed by $\Omega \subset \mathbb{R}^{d}$ with values in $\mathbb{R}^{N_{u}}$, for which its cross-covariance function is square integrable $\Omega \times \Omega$, then a reduced representation $\mathbf{u}^{(m)}(\mathbf{x})=\sum_{i=1}^{m} \eta_{i} \sqrt{\lambda_{i}} \mathbf{b}^{i}(\mathbf{x})$ of $\mathbf{u}$ can be constructed using the Karhunen-Loève expansion of $\mathbf{u}$, in which $m$ is calculated in order that the mean-square error of $\mathbf{u}-\mathbf{u}^{(m)}$ is sufficiently small. Therefore, the explanations given before can be applied to the random vector $\boldsymbol{\eta}=\left(\eta_{1}, \ldots, \eta_{m}\right)$ in order to estimate the stochastic dimension of random field $\mathbf{u}$.

What is a parametric model-based representation for the statistical identification of a random model parameter from experimental data?

In order to simply explain what is a parametric model-based representation for the statistical identification of a random model parameter from experimental data, let us consider the stochastic elliptic boundary value problem formulated for a realvalued random field $u(\mathbf{x})$ indexed by $\mathbf{x}=\left(x_{1}, \ldots, x_{d}\right)$ belonging to a subset $\Omega$ of $\mathbb{R}^{d}$, and which is assumed to have a unique second-order stochastic solution $u$. The stochastic elliptic operator of the boundary value problem is written as $-\sum_{j=1}^{d} \frac{\partial}{\partial x_{j}}\left\{K(\mathbf{x}) \frac{\partial}{\partial x_{j}} u(\mathbf{x})\right\}$ in which the random field $K=\{K(\mathbf{x}), \mathbf{x} \in \Omega\}$, indexed by $\Omega$, with values in $\mathbb{R}^{+}=[0,+\infty[$ is defined as the model parameter of the boundary value problem. Let $\mathbf{U}$ be a random model observation that is assumed to be a random vector with values in $\mathbb{R}^{N_{U}}$, which is deduced from random field $u$ by a deterministic observation operator $\mathcal{O}$, such that $\mathbf{U}=\mathcal{O}(u)$. Consequently, random model observation $\mathbf{U}$ can be written as $\mathbf{U}=\mathcal{H}(K)$ in which $\mathcal{H}$ is a deterministic nonlinear functional of $K$. For all $\mathbf{x}$ in $\Omega$, a representation of $K$ is assumed to be written as $K(\mathbf{x})=\mathcal{G}(G(\mathbf{x}))$ with $G(\mathbf{x})=G_{0}(\mathbf{x})+\sum_{i=1}^{m} \eta_{i} \sqrt{\lambda_{i}} G_{i}(\mathbf{x})$. The deterministic nonlinear mapping $\mathcal{G}$ is independent of $\mathbf{x}$ and is assumed to be from $\mathbb{R}$ into $\mathbb{R}^{+}$. With the introduction of such a deterministic mapping $\mathcal{G}$, for all $\mathbf{x}$ fixed in $\Omega$, the support of the probability distribution of the random variable $G(\mathbf{x})$ 
is $\mathbb{R}$ instead of $\mathbb{R}^{+}$for $K(\mathbf{x})$. In the reduced representation of the random field $G$ indexed by $\Omega$, with values in $\mathbb{R}$, the quantities $G_{0}(\mathbf{x}), \lambda_{i}$, and $G_{i}(\mathbf{x})$ are some real numbers. The random vector $\boldsymbol{\eta}=\left(\eta_{1}, \ldots, \eta_{m}\right)$ is written as $\left.\boldsymbol{\eta}=\mathcal{Y}(\boldsymbol{\Xi} ; z]\right)$ in which $\Xi=\left(\Xi_{1}, \ldots, \Xi_{N_{g}}\right)$ is a given vector-valued random variable, where $\mathcal{Y}$ is a deterministic nonlinear mapping representing the truncated polynomial chaos expansion of $\boldsymbol{\eta}$ with respect to $\Xi$, and where $[z]$ is the real matrix of the $\mathbb{R}^{m}$ valued coefficients of the truncated polynomial chaos expansion of $\boldsymbol{\eta}$. It can then be deduced that random model observation $\mathbf{U}$ can be rewritten as $\mathbf{U}=\mathcal{B}(\boldsymbol{\Xi},[z])$ in which $\mathcal{B}$ is a deterministic nonlinear mapping depending on $\mathcal{H}, \mathcal{G}$ and $\mathcal{Y}$. This last representation is defined as a parametric model-based representation of the random model observation $\mathbf{U}$ in which the real matrix $[z]$ is the hyperparameter of the representation. Let us assume that some experimental data $\mathbf{u}^{\exp , 1}, \ldots, \mathbf{u}^{\exp , \nu_{\exp }}$ related to random model observation $\mathbf{U}$ are available. The identification of the model parameter $K$ using the experimental data consists in identifying the real matrix $[z]$ using the parametric model-based representation $\mathbf{U}=\mathcal{B}(\boldsymbol{\Xi},[z])$ of the random model observation and the corresponding experimental data.

\section{Brief History}

\section{Classical methods for statistical inverse problems.}

The problem related to the identification of a model parameter (scalar, vector, field) of a boundary value problem (BVP) (for instance, the coefficients of a partial differential equation) using experimental data related to a model observation (scalar, vector, field) of this BVP, is a problem for which there exists a rich literature, including numerous textbooks. In general and in the deterministic context, there is not a unique solution because the function, which maps the model parameter (that belongs to an admissible set) to the model observation (that belongs to another admissible set) is not a one-to-one mapping, and consequently, cannot be inverted. It is an ill-posed problem. However, such a problem can be reformulated in terms of an optimization problem consisting in calculating an optimal value of the model parameter, which minimizes a certain distance between the observed experimental data and the model observation that is computed with the BVP and that depends on the model parameter (see for instance [76] for an overview concerning the general methodologies, and [36] for some mathematical aspects related to the inverse problems for partial differential equations). In many cases, the analysis of such an inverse problem can have a unique solution in the framework of statistics, that is to say when the model parameter is modeled by a random quantity, with or without external noise on the model observation (observed output). In such a 
case, the random model observation is completely defined by its probability distribution (in finite or in infinite dimension) that is the unique transformation of the probability distribution of the random model parameter. This transformation is defined by the functional that maps the model parameter to the model observation. Such a formulation is constructed for obtaining a well-posed problem that has a unique solution in the probability theory framework. We refer the reader to [38] and [72] for an overview concerning the general methodologies for statistical and computational inverse problems, including general least-square inversion and the maximum likelihood method [54;67], and including the Bayesian approach $[67 ; 9 ; 8 ; 68]$.

\section{Case of a Gaussian random model parameter.}

A Gaussian second-order random vector is completely defined by its second-order moments, that is to say, by its mean vector and by its covariance matrix. Similarly, a Gaussian second-order random field is completely defined by its mean function and by its cross-covariance function or, if the random field is homogeneous (stationary) and mean-square continuous, by its spectral measure [17]. If the model parameter is Gaussian (random vector or random field), then the statistical inverse problem (identification of the system parameter using experimental data related to the model observation of the system) consists in identifying the second-order moments, which is relatively easy for a low or a high stochastic dimension. Concerning the description of the Gaussian random fields, we refer the reader to the abundant existing literature (see for instance $[41 ; 53 ; 74]$ ).

\section{Case for which the model parameter is a non-Gaussian second-order random field.}

A non-Gaussian second-order random field is completely defined by its system of marginal probability distributions, which is an uncountable family of probability distributions on sets of finite dimension, and not only by its mean function and its covariance function as for a Gaussian random field. The experimental identification of such a non-Gaussian random field then requires the introduction of an adapted representation in order to be in capability to solve the statistical inverse problem. For any non-Gaussian second-order random field, an important type of representation is based on the use of the polynomial chaos expansion [7], for which the development and the use in computational sciences and engineering have been pioneered by Roger Ghanem in 1990-1991 [21]. An efficient construction is proposed, which consists in combining a Karhunen-Loève expansion (that allows using a statistical reduced model) with a polynomial chaos expansion of 
the statistical reduced model. This type of construction has then been re-analyzed and used for solving boundary value problems using the spectral approach (see for instance $[52 ; 13 ; 18 ; 24 ; 25 ; 46 ; 43 ; 47]$. The polynomial chaos expansion as also been extended for an arbitrary probability measure $[78 ; 42 ; 44 ; 57 ; 77 ; 20]$ and for sparse representation [5]. New algorithms have been proposed for obtaining a robust computation of realizations of high degrees polynomial chaos [63;49]. This type of representation has also been extended for the case of the polynomial chaos expansion with random coefficients [61], for the construction of a basis adaptation in homogeneous chaos spaces [73], and for an arbitrary multimodal multidimensional probability distribution [66].

Finite-dimension approximation of the BVP and finite-dimension parameterization of the random field.

A finite-dimension parameterized representation of the non-Gaussian random field must be constructed in order to be able to solve the statistical inverse problem. In addition and in general, an explicit solution of the BVP cannot be obtained and consequently, a finite-dimension approximation of the solution of the BVP must also be constructed (using for instance the finite element method), accompanied by a convergence analysis. The combination of these two approximations leads us to introduce a non-Gaussian second-order random vector $\boldsymbol{\eta}$ with values in $\mathbb{R}^{m}$, which is the finite-dimension parameterized representation of the random model parameter of the system. Consequently, the statistical inverse problem consists in identifying the non-Gaussian second-order random vector $\boldsymbol{\eta}$ that is completely defined by its probability distribution on $\mathbb{R}^{m}$. Nevertheless, as $\boldsymbol{\eta}$ corresponds to a finite-dimension parameterization of the finite discretization of a random field, it is necessary to construct, first, a good mathematical representation of the random field and of its finite-dimension parameterization, before performing its spatial discretization.

\section{Parameterization of the non-Gaussian second-order random vector $\boldsymbol{\eta}$.}

Since it is assumed that the experimental data that are available for the statistical inverse problem are partial and limited, the parametric statistics must be used instead of the nonparametric statistics that cannot be used. This implies that a parameterized representation of the non-Gaussian second-order random vector $\boldsymbol{\eta}$ must be constructed. There are two main methods for constructing such a parameterization.

(i) The first one is a direct approach that consists in constructing a algebraic prior representation of the non-Gaussian probability distribution of $\boldsymbol{\eta}$ in using the 
maximum entropy principle (MaxEnt) [37; 65] under the constraints defined by the available information. A general computational methodology, for the problems in high stochastic dimension, is proposed in [59;4] and is synthesized in Section "MaxEnt for Constructing the pdf of a Random Vector" of "Random Matrix Models and Nonparametric Method for Uncertainty Quantification" in part II of the present Handbook on Uncertainty Quantification. Such a construction allows a low-dimension hyperparameterization to be obtained for the non-Gaussian probability distribution on $\mathbb{R}^{m}$. Therefore, the parametric statistics $[76 ; 54 ; 67]$ can be used for solving the statistical inverse problem consisting in identifying the vector-valued hyperparameter of the probability distribution constructed with the MaxEnt. In counter part, the "distance" between the observed experimental data and the random model observation cannot be, in general, reduced to zero. A residual error exists. If there are a sufficient amount of experimental data, this error can be reduced by identifying a posterior probability distribution of $\boldsymbol{\eta}$ using the Bayesian approach $[67 ; 9 ; 8]$.

(ii) The second method is an indirect approach which consists in introducing a representation $\boldsymbol{\eta}=\mathcal{Y}(\boldsymbol{\Xi})$ in which $\mathcal{Y}$ is an unknown deterministic nonlinear (measurable) mapping from $\mathbb{R}^{N_{g}}$ into $\mathbb{R}^{m}$ (which has to be constructed) and where $\Xi$ is a given random vector with values in $\mathbb{R}^{N_{g}}$, for which its probability distribution is known (for instance a normalized Gaussian random vector). The statistical inverse problem then consists in identifying the nonlinear mapping $\mathcal{Y}$. Consequently, a parameterization of mapping $\mathcal{Y}$ must be introduced in order to use parametric statistics, and there are two main approaches.

(ii.1) The first one corresponds to the truncated polynomial chaos expansion of second-order random vector $\boldsymbol{\eta}$ with respect to the normalized Gaussian measure. In this case, $\boldsymbol{\Xi}$ is a normalized Gaussian random vector and the orthogonal polynomials are the normalized Hermite polynomials [21]). If an arbitrary probability measure is used instead of the normalized Gaussian measure, then $\Xi$ is a normalized random vector with this arbitrary probability distribution, and the orthogonal polynomials are constructed with respect to this arbitrary probability distribution $[78 ; 57 ; 77 ; 49 ; 66]$. Such a polynomial expansion defines a parameterization, noted as $\mathcal{Y}(\boldsymbol{\Xi},[z])$, of mapping $\mathcal{Y}$, in which the real matrix $[z]^{T}$ represents the $\mathbb{R}^{m}$-valued coefficients of the polynomial chaos expansion of $\boldsymbol{\eta}$, and the identification of $\mathcal{Y}$ is replaced by the identification of the hyperparameter $[z]$.

(ii.2) The second approach consists in introducing an algebraic prior representation $\boldsymbol{\eta}=\mathcal{Y}(\boldsymbol{\Xi}, \mathbf{s})$ in which $\mathbf{s}$ is a vector-valued hyperparameter that has a small dimension, and which must be identified using parametric statistics [76; 54; 67]. Similarly to the method (i) presented before, if there is a sufficient amount of ex- 
perimental data, the prior model can be updated in constructing a posterior probability distribution using the Bayesian approach [23; 45].

\section{Statistical inverse problem for identifying a non-Gaussian random field as a model parameter of a BVP, using polynomial chaos expansion.}

The use of the polynomial chaos expansion for constructing a parameterized representation of a non-Gaussian random field that models the model parameter of a boundary value problem, in order to identify it using a statistical inverse method has been initialized in [14;15], used in [26], and revisited in [12]. In [10], the construction of the probability model of the random coefficients of the polynomial chaos expansion is proposed by using the asymptotic sampling Gaussian distribution constructed with the Fisher information matrix, and used for model validation [24]. This work has been developed for statistical inverse problems that are rather in low stochastic dimension, and new ingredients have been introduced in $[62 ; 49 ; 65]$ for statistical inverse problems in high stochastic dimension. In using the reduced chaos decomposition with random coefficients of random fields [61], a Bayesian approach for identifying the posterior probability model of the random coefficients of the polynomial chaos expansion of the model parameter of the BVP has been proposed in [2] for the low stochastic dimension and in [64] for the high stochastic dimension. The experimental identification of a nonGaussian positive matrix-valued random field in high stochastic dimension, using partial and limited experimental data for a model observation related to the random solution of a stochastic BVP, is a difficult problem that requires both adapted representations and methodologies $[62 ; 64 ; 65 ; 48]$.

\section{Algebraic prior stochastic models of the model parameters of BVP.}

In the methodology devoted to the identification of a non-Gaussian random field in high stochastic dimension, an important step is the construction of a parameterized representation for which the number of hyperparameters (in the parameterized representation) is generally very large due to the high stochastic dimension. In the framework of hypotheses for which only partial and limited data are available, such an identification is difficult if there is no information concerning the region of the admissible set (in high dimension), in which the optimal values of these hyperparameters must be searched. The optimization process, related to the statistical inverse problem, requires to localize the region in which the algorithms must search for an optimal value. The method consists in previously identifying the "center" of such a region, which corresponds to the value of the hyperparameters of the parameterized representation using a set of realizations generated with 
an algebraic prior stochastic model (APSM) that is specifically constructed on the basis of the available information associated with all the mathematical properties of the non-Gaussian random field that has to be identified. This APSM allows for enriching the information in order to overcome the lack of experimental data (since only partial experimental data are assumed to be available). This is particularly crucial for the identification of the non-Gaussian matrix-valued random field encountered, for instance, in three-dimensional linear elasticity, for which some works have been performed in order to introduce the symmetry, the positiveness and invertibility properties $[56 ; 58 ; 60]$, the boundedness $[28 ; 30]$, a capability of the prior stochastic model to exhibit a capability to generate simultaneously anisotropic statistical fluctuations and some statistical fluctuations in a symmetry class such as isotropic, cubic, transversely isotropic, orthotropic, etc. $[69 ; 29 ; 31 ; 32 ; 34]$, and to develop the corresponding generators of realizations $[58 ; 62 ; 29 ; 32 ; 34]$.

\section{Overview}

A complete methodology and the associated tools are presented for the experimental identification of a non-Gaussian matrix-valued random field that is the model parameter of a boundary value problem, using some experimental data related to a model observation. The difficulties of the statistical inverse problem that are presented are due to the following chosen framework that corresponds to many practical situations in computational sciences and engineering:

- A non-Gaussian matrix-valued random field must be identified, not simply a real-valued random field.

- The non-Gaussian random field that has to be identified is in high stochastic dimension and must be identified in a general class of random fields.

- Some fundamental algebraic properties of the non-Gaussian random field must be satisfied such as symmetry, positiveness, invertibility in mean square, boundedness, symmetry class, spatial-correlation lengths, etc.

- The available experimental data sets correspond only to partial and limited data for a model observation of the boundary value problem.

For such a statistical inverse problem, the above framework implies the use of an adapted and advanced methodology. The developments presented hereinafter are mainly related to the elasticity framework, but the methodology is general and can be used in many areas of computational sciences and engineering. The developments are organized as follows. 
- The first one is devoted to the definition of the statistical inverse problem that has to be solved in high stochastic dimension, and is focussed on stochastic elliptic operators such as the ones that are encountered in the boundary value problems of the linear elasticity.

- The second one deals with the construction of two possible parameterized representations for a non-Gaussian positive-definite matrix-valued random field that models the model parameter of a boundary value problem. A parametric model-based representation is then constructed in introducing a statistical reduced model and a polynomial chaos expansion, first with deterministic coefficients and after with random coefficients. This parametric model-based representation is directly used for solving the statistical inverse problem.

- The third part is devoted to the description of all the steps of the methodology allowing the statistical inverse problem to be solved in high stochastic dimension. This methodology corresponds to the work initialized in [62], extended in [64] for constructing a posterior stochastic model using the Bayesian approach, and revisited in [49; 48].

- The fourth part presents the construction of an algebraic prior stochastic model of the model parameter of the boundary value problem, for a non-Gaussian matrix-valued random field. This construction is based on the works [58;60; $30 ; 32]$, and reuses the formalism and the results introduced in the developments presented in Section "Nonparametric Stochastic Model For Constitutive Equation in Linear Elasticity" of "Random Matrix Models and Nonparametric Method for Uncertainty Quantification" in part II of the present Handbook on Uncertainty Quantification. The generator of realizations for such an algebraic prior stochastic model for a non-Gaussian matrix-valued random field is presented [58; 62; 32].

\section{Notations}

The following algebraic notations are used.

\section{Euclidean space.}

Let $\mathbf{x}=\left(x_{1}, \ldots, x_{n}\right)$ be a vector in $\mathbb{R}^{n}$. The Euclidean space $\mathbb{R}^{n}$ is equipped with the usual inner product $<\mathbf{x}, \mathbf{y}>=\sum_{j=1}^{n} x_{j} y_{j}$ and the associated norm $\|\mathbf{x}\|=<$ $\mathbf{x}, \mathbf{x}>^{1 / 2}$.

\section{Sets of matrices.}

$\mathbb{M}_{n, m}(\mathbb{R})$ be the set of all the $(n \times m)$ real matrices, $\mathbb{M}_{n}(\mathbb{R})=\mathbb{M}_{n, n}(\mathbb{R})$ the square matrices, 
$\mathbb{M}_{n}^{S}(\mathbb{R})$ be the set of all the symmetric $(n \times n)$ real matrices,

$\mathbb{M}_{n}^{U}(\mathbb{R})$ be the set of all the upper triangular $(n \times n)$ real matrices with positive diagonal entries,

$\mathbb{M}_{n}^{+}(\mathbb{R})$ be the set of all the positive-definite symmetric $(n \times n)$ real matrices. The ensembles of real matrices are such that

$\mathbb{M}_{n}^{+}(\mathbb{R}) \subset \mathbb{M}_{n}^{S}(\mathbb{R}) \subset \mathbb{M}_{n}(\mathbb{R})$.

Kronecker symbol, unit matrix, and indicator function.

The Kronecker symbol is denoted by $\delta_{j k}$ and is such that $\delta_{j k}=0$ if $j \neq k$ and $\delta_{j j}=1$. The unit (or identity) matrix in $\mathbb{M}_{n}(\mathbb{R})$ is denoted by $\left[I_{n}\right]$ and is such that $\left[I_{n}\right]_{j k}=\delta_{j k}$. Let $\mathbb{S}$ be any subset of any set $\mathbb{M}$, possibly with $\mathbb{S}=\mathbb{M}$. The indicator function $M \mapsto \mathbb{1}_{\mathbb{S}}(M)$ defined on set $\mathbb{M}$ is such that $\mathbb{1}_{\mathbb{S}}(M)=1$ if $M \in \mathbb{S} \subset \mathbb{M}$, and $\mathbb{1}_{\mathbb{S}}(M)=0$ if $M \notin \mathbb{S}$.

\section{Norms and usual operators.}

(i) The determinant of a matrix $[G]$ in $\mathbb{M}_{n}(\mathbb{R})$ is denoted by $\operatorname{det}[G]$, and its trace is denoted by $\operatorname{tr}[G]=\sum_{j=1}^{n} G_{j j}$.

(ii) The transpose of a matrix $[G]$ in $\mathbb{M}_{n, m}(\mathbb{R})$ is denoted by $[G]^{T}$, which is in $\mathbb{M}_{m, n}(\mathbb{R})$.

(iii) The operator norm of a matrix $[G]$ in $\mathbb{M}_{n, m}(\mathbb{R})$ is denoted by $\|G\|=\sup _{\|\mathbf{x}\| \leq 1}$ $\|[G] \mathbf{x}\|$ for all $\mathbf{x}$ in $\mathbb{R}^{m}$, which is such that $\|[G] \mathbf{x}\| \leq\|G\|\|\mathbf{x}\|$ for all $\mathbf{x}$ in $\mathbb{R}^{m}$.

(iv) For $[G]$ and $[H]$ in $\mathbb{M}_{n, m}(\mathbb{R})$, we denote $\ll[G],[H] \gg=\operatorname{tr}\left\{[G]^{T}[H]\right\}$ and the Frobenius norm (or Hilbert-Schmidt norm) $\|G\|_{F}$ of $[G]$ is such that $\|G\|_{F}^{2}=\ll$ $[G],[G] \gg=\operatorname{tr}\left\{[G]^{T}[G]\right\}=\sum_{j=1}^{n} \sum_{k=1}^{m} G_{j k}^{2}$, which is such that $\|G\| \leq\|G\|_{F} \leq$ $\sqrt{n}\|G\|$.

(v) The gradient $\nabla_{\mathbf{x}} u(\mathbf{x})$ at point $\mathbf{x}$ in $\mathbb{R}^{n}$ of the real-valued function $\mathbf{x} \mapsto u(\mathbf{x})$, is the vector in $\mathbb{R}^{n}$ such that $\left\{\boldsymbol{\nabla}_{\mathbf{x}} u(\mathbf{x})\right\}_{j}=\partial u(\mathbf{x}) / \partial x_{j}$ for $j=1, \ldots, n$. The divergence $\operatorname{div}_{\mathbf{x}}(\mathbf{u}(\mathbf{x}))$ at point $\mathbf{x}$ in $\mathbb{R}^{n}$ of the $\mathbb{R}^{n}$-valued function $\mathbf{x} \mapsto \mathbf{u}(\mathbf{x})=$ $\left(u_{1}(\mathbf{x}), \ldots, u_{n}(\mathbf{x})\right)$, is the real number such that $\operatorname{div}_{\mathbf{x}}(\mathbf{u}(\mathbf{x}))=\sum_{j=1}^{n} \partial u_{j}(\mathbf{x}) / \partial x_{j}$.

Order relation in the set of all the positive-definite real matrices.

Let $[G]$ and $[H]$ be two matrices in $\mathbb{M}_{n}^{+}(\mathbb{R})$. The notation $[G]>[H]$ means that the matrix $[G]-[H]$ belongs to $\mathbb{M}_{n}^{+}(\mathbb{R})$.

Probability space, mathematical expectation, space of second-order random vectors.

The mathematical expectation relative to a probability space $(\Theta, \mathcal{T}, P)$ is denoted 
by $E$. The space of all the second-order random variables, defined on $(\Theta, \mathcal{T}, P)$, with values in $\mathbb{R}^{n}$, equipped with the inner product $((\mathbf{X}, \mathbf{Y}))=E\{<\mathbf{X}, \mathbf{Y}>\}$ and with the associated norm $\|\mid \mathbf{X}\| \|=((\mathbf{X}, \mathbf{X}))^{1 / 2}$, is a Hilbert space denoted by $\mathcal{L}_{n}^{2}$

\section{Setting the Statistical Inverse Problem to Be Solved in High Stochastic Dimension}

Let $d$ be an integer such that $1 \leq d \leq 3$. Let $n$ be another finite integer such that $n \geq 1$, and let $N_{u}$ be an integer such that $1 \leq N_{u} \leq n$. Let $\Omega$ be a bounded open domain of $\mathbb{R}^{d}$, with generic point $\mathbf{x}=\left(x_{1}, \ldots, x_{d}\right)$, with boundary $\partial \Omega$, and let be $\bar{\Omega}=\Omega \cup \partial \Omega$.

Stochastic elliptic operator and boundary value problem.

Let $[\mathbf{K}]=\{[\mathbf{K}(\mathbf{x})], \mathbf{x} \in \Omega\}$ be a non-Gaussian random field, in high stochastic dimension, defined on a probability space $(\Theta, \mathcal{T}, \mathcal{P})$, indexed by $\Omega$, with values in $\mathbb{M}_{n}^{+}(\mathbb{R})$. It should be noted that random field $[\mathbf{K}]$ being with values in $\mathbb{M}_{n}^{+}(\mathbb{R})$, random field $[\mathbf{K}]$ cannot be a Gaussian field. Such a random field $[\mathbf{K}]$ allows for constructing the coefficients of a given stochastic elliptic operator $\mathbf{u} \mapsto \mathcal{D}_{\mathbf{x}}(\mathbf{u})$ that applies to the random field $\mathbf{u}(\mathbf{x})=\left(u_{1}(\mathbf{x}), \ldots, u_{N_{u}}(\mathbf{x})\right)$, indexed by $\Omega$, with values in $\mathbb{R}^{N_{u}}$.

The boundary value problem that is formulated in $\mathbf{u}$, involves the stochastic elliptic operator $\mathcal{D}_{\mathbf{x}}$, and some Dirichlet and Neumann boundary conditions are given on $\partial \Omega$ that is written as the union of three parts, $\partial \Omega=\Gamma_{0} \cup \Gamma \cup \Gamma_{1}$. On the part $\Gamma_{0}$, a Dirichlet condition is given. The part $\Gamma$ corresponds to the part of the boundary on which there is a zero Neumann condition and on which experimental data are available for $\mathbf{u}$. On the part $\Gamma_{1}$, a Neumann condition is given. The boundary value problems, involving such a stochastic elliptic operator $\mathcal{D}_{\mathbf{x}}$, are encountered in many problems of computational sciences and engineering.

- Examples of stochastic elliptic operators.

(i) For a three-dimensional anisotropic diffusion problem, the stochastic elliptic differential operator $\mathcal{D}_{\mathbf{x}}$ relative to the density $u$ of the diffusing medium, is written as

$$
\left\{\mathcal{D}_{\mathbf{x}}(u)\right\}(\mathbf{x})=-\operatorname{div}_{\mathbf{x}}\left([\mathbf{K}(\mathbf{x})] \boldsymbol{\nabla}_{\mathbf{x}} u(\mathbf{x})\right) \quad, \quad \mathbf{x} \in \Omega,
$$

in which $d=n=3$ and $N_{u}=1$, and where $\{[\mathbf{K}(\mathbf{x})], \mathbf{x} \in \Omega\}$ is the $\mathbb{M}_{n}^{+}(\mathbb{R})$-valued random field of the medium. 
(ii) For the wave propagation inside a three-dimensional random heterogeneous anisotropic linear elastic medium, we have $d=3, n=6, N_{u}=3$, and the stochastic elliptic differential operator $\mathcal{D}_{\mathbf{x}}$ relative to the displacement field $\mathbf{u}$ is written as

$$
\left\{\mathcal{D}_{\mathbf{x}}(\mathbf{u})\right\}(\mathbf{x})=-\left[D_{\mathbf{x}}\right]^{T}[\mathbf{K}(\mathbf{x})]\left[D_{\mathbf{x}}\right] \mathbf{u}(\mathbf{x}) \quad, \quad \mathbf{x} \in \Omega,
$$

in which $\{[\mathbf{K}(\mathbf{x})], \mathbf{x} \in \Omega\}$ is the $\mathbb{M}_{n}^{+}(\mathbb{R})$-valued elasticity random field of the medium deduced from the fourth-order tensor-valued elasticity field $\left\{\mathbf{C}_{i j k h}(\mathbf{x}), \mathbf{x} \in\right.$ $\Omega$ \} by the following equation,

$$
[\mathbf{K}]=\left[\begin{array}{cccccc}
\mathbf{C}_{1111} & \mathbf{C}_{1122} & \mathbf{C}_{1133} & \sqrt{2} \mathbf{C}_{1112} & \sqrt{2} \mathbf{C}_{1113} & \sqrt{2} \mathbf{C}_{1123} \\
\mathbf{C}_{2211} & \mathbf{C}_{2222} & \mathbf{C}_{2233} & \sqrt{2} \mathbf{C}_{2212} & \sqrt{2} \mathbf{C}_{2213} & \sqrt{2} \mathbf{C}_{2223} \\
\mathbf{C}_{3311} & \mathbf{C}_{3322} & \mathbf{C}_{3333} & \sqrt{2} \mathbf{C}_{3312} & \sqrt{2} \mathbf{C}_{3313} & \sqrt{2} \mathbf{C}_{3323} \\
\sqrt{2} \mathbf{C}_{1211} & \sqrt{2} \mathbf{C}_{1222} & \sqrt{2} \mathbf{C}_{1233} & 2 \mathbf{C}_{1212} & 2 \mathbf{C}_{1213} & 2 \mathbf{C}_{1223} \\
\sqrt{2} \mathbf{C}_{1311} & \sqrt{2} \mathbf{C}_{1322} & \sqrt{2} \mathbf{C}_{1333} & 2 \mathbf{C}_{1312} & 2 \mathbf{C}_{1313} & 2 \mathbf{C}_{1323} \\
\sqrt{2} \mathbf{C}_{2311} & \sqrt{2} \mathbf{C}_{2322} & \sqrt{2} \mathbf{C}_{2333} & 2 \mathbf{C}_{2312} & 2 \mathbf{C}_{2313} & 2 \mathbf{C}_{2323}
\end{array}\right],
$$

in which $\left[D_{\mathbf{x}}\right]$ is the differential operator,

$$
\left[D_{\mathbf{x}}\right]=\left[M^{(1)}\right] \frac{\partial}{\partial x_{1}}+\left[M^{(2)}\right] \frac{\partial}{\partial x_{2}}+\left[M^{(3)}\right] \frac{\partial}{\partial x_{3}},
$$

where $\left[M^{(1)}\right],\left[M^{(2)}\right]$ and $\left[M^{(3)}\right]$ are the $\left(n \times N_{u}\right)$ real matrices defined by

$$
\left[M^{(1)}\right]=\left[\begin{array}{ccc}
1 & 0 & 0 \\
0 & 0 & 0 \\
0 & 0 & 0 \\
0 & \frac{1}{\sqrt{2}} & 0 \\
0 & 0 & \frac{1}{\sqrt{2}} \\
0 & 0 & 0
\end{array}\right],\left[M^{(2)}\right]=\left[\begin{array}{ccc}
0 & 0 & 0 \\
0 & 1 & 0 \\
0 & 0 & 0 \\
\frac{1}{\sqrt{2}} & 0 & 0 \\
0 & 0 & 0 \\
0 & 0 & \frac{1}{\sqrt{2}}
\end{array}\right],\left[M^{(3)}\right]=\left[\begin{array}{ccc}
0 & 0 & 0 \\
0 & 0 & 0 \\
0 & 0 & 1 \\
0 & 0 & 0 \\
\frac{1}{\sqrt{2}} & 0 & 0 \\
0 & \frac{1}{\sqrt{2}} & 0
\end{array}\right] .
$$

- Example of a time-independent stochastic boundary value problem in linear elasticity.

Let be $d=3, n=6$, and $N_{u}=3$. Let us consider the boundary value problem related to the linear elastostatic deformation of a three-dimensional random heterogeneous anisotropic linear elastic medium occupying domain $\Omega$, for which an experimental displacement field $\mathbf{u}^{\exp , \ell}$ is measured on $\Gamma$. Let $\mathbf{n}(\mathbf{x})=$ 
$\left(n_{1}(\mathbf{x}), n_{2}(\mathbf{x}), n_{3}(\mathbf{x})\right)$ be the unit normal to $\partial \Omega$, exterior to $\Omega$. The stochastic boundary value problem is written as

$$
\mathcal{D}_{\mathbf{x}}(\mathbf{u})=\mathbf{0} \quad \text { in } \Omega,
$$

in which the stochastic operator $\mathcal{D}_{\mathbf{x}}$ is defined by Eq. (2), where the Dirichlet condition is

$$
\mathbf{u}=\mathbf{0} \quad \text { on } \Gamma_{0},
$$

and where the Neumann condition is written as

$$
\left[\mathcal{M}_{\mathbf{n}}(\mathbf{x})\right]^{T}[\mathbf{K}(\mathbf{x})]\left[D_{\mathbf{x}}\right] \mathbf{u}(\mathbf{x})=\mathbf{0} \text { on } \Gamma \text {, and }=\mathbf{f}_{\Gamma_{1}} \text { on } \Gamma_{1},
$$

in which $\left[\mathcal{M}_{\mathbf{n}}(\mathbf{x})\right]=\left[M^{(1)}\right] n_{1}(\mathbf{x})+\left[M^{(2)}\right] n_{2}(\mathbf{x})+\left[M^{(3)}\right] n_{3}(\mathbf{x})$, and where $\mathbf{f}_{\Gamma_{1}}$ is a given surface force field applied to $\Gamma_{1}$. The boundary value problem defined by Eqs. (6) to (8) is typically the one for which the random field $\{[\mathbf{K}(\mathbf{x}), \mathbf{x} \in \Omega\}$ has to be identified by solving a statistical inverse problem in high stochastic dimension with the partial and limited experimental data $\left\{\mathbf{u}^{\exp , \ell}, \ell=1, \ldots, \nu_{\exp }\right\}$.

\section{Stochastic finite element approximation of the stochastic boundary value prob- lem.}

Let us assume that the weak formulation of the stochastic boundary value problem involving stochastic elliptic operator $\mathcal{D}_{\mathbf{x}}$, is discretized by using the finite element method. Let $\mathcal{I}=\left\{\mathbf{x}^{1}, \ldots, \mathbf{x}^{N_{p}}\right\} \subset \Omega$ be the finite subset of $\Omega$ made up of all the integrating points in the numerical integration formulae for the finite elements [79] used in the mesh of $\Omega$. Let $\mathbf{U}=\left(U_{1}, \ldots, U_{N_{U}}\right)$ be the random model observation with values in $\mathbb{R}^{N_{U}}$, constituted of the $N_{U}$ observed degrees of freedom for which there are available experimental data (corresponding to some degrees of freedom of the nodal values of $\mathbf{u}$ at all the nodes in $\Gamma$ ). The random observation vector $\mathbf{U}$ is the unique deterministic nonlinear transformation of the finite family of the $N_{p}$ dependent random matrices $\left[\mathbf{K}\left(\mathbf{x}^{1}\right)\right], \ldots,\left[\mathbf{K}\left(\mathbf{x}^{N_{p}}\right)\right]$ such that

$$
\mathbf{U}=\mathbf{h}\left(\left[\mathbf{K}\left(\mathbf{x}^{1}\right)\right], \ldots,\left[\mathbf{K}\left(\mathbf{x}^{N_{p}}\right)\right]\right),
$$

in which

$$
\left.\left(\left[K^{1}\right], \ldots,\left[K^{N_{p}}\right)\right]\right) \mapsto \mathbf{h}\left(\left[K^{1}\right], \ldots,\left[K^{N_{p}}\right)\right): \mathbb{M}_{n}^{+}(\mathbb{R}) \times \ldots \times \mathbb{M}_{n}^{+}(\mathbb{R}) \longrightarrow \mathbb{R}^{N_{U}}
$$

is a deterministic nonlinear transformation that is constructed by solving the discretized boundary value problem. 


\section{Experimental data sets.}

It is assumed that $\nu_{\text {exp }}$ experimental data sets are available for the random observation vector $\mathbf{U}$. Each experimental data set corresponds to partial experimental data (only some degrees of freedom of the nodal values of the displacement field on $\Gamma$ are observed) with a limited length ( $\nu_{\exp }$ is relatively small). These $\nu_{\exp }$ experimental data sets correspond to measurements of $\nu_{\exp }$ experimental configurations associated with the same boundary value problem. For configuration $\ell$, with $\ell=1, \ldots, \nu_{\text {exp }}$, the observation vector (corresponding to $\mathbf{U}$ for the computational model) is denoted by $\mathbf{u}^{\exp , \ell}$ and belongs to $\mathbb{R}^{N_{U}}$. Therefore, the available data are made up of the $\nu_{\exp }$ vectors $\mathbf{u}^{\exp , 1}, \ldots, \mathbf{u}^{\exp , \nu_{\exp }}$ in $\mathbb{R}^{N_{U}}$. It is assumed that $\mathbf{u}^{\exp , 1}, \ldots, \mathbf{u}^{\exp , \nu_{\exp }}$ correspond to $\nu_{\exp }$ independent realizations of a random vector $\mathbf{U}^{\exp }$ defined on a probability space $\left(\Theta^{\exp }, \mathcal{T}^{\exp }, \mathcal{P}^{\exp }\right)$ and corresponding to random observation vector $\mathbf{U}$ of the stochastic computational model (random vectors $\mathbf{U}^{\exp }$ and $\mathbf{U}$ are not defined on the same probability space). It should be noted that the experimental data do not correspond to a field measurement in $\bar{\Omega}$, but only to a field measurement on the part $\Gamma$ of the boundary $\partial \Omega$ of domain $\Omega$. This is the reason why the experimental data are called "partial".

\section{Statistical inverse problem to be solved.}

The problem that must be solved is the identification of non-Gaussian matrixvalued random field $[\mathbf{K}]$, using the partial and limited experimental data $\mathbf{u}^{\exp , 1}$, $\ldots, \mathbf{u}^{\exp , \nu_{\exp }}$ relative to the random observation vector $\mathbf{U}$ of the stochastic computational model and defined by Eq. (9).

\section{Parametric Model-Based Representation for the Model Parameters and Model Observations}

As explained in the previous paragraph entitled "What is a parametric modelbased representation for the statistical identification of a random model parameter from experimental data?, a parametric model-based representation $\mathbf{U}=\mathcal{B}(\boldsymbol{\Xi}$, $[z])$ must be constructed in order to be able to solve the statistical inverse problem allowing random model parameter $[\mathbf{K}]$ to be identified using the experimental data sets. For that, it is needed to introduce

- a representation of the non-Gaussian positive-definite matrix-valued random field $[\mathbf{K}]$ that is expressed as a transformation $\mathcal{G}$ of a non-Gaussian second-order symmetric matrix-valued random field $[\mathbf{G}]$, such that for all $\mathbf{x}$ in $\Omega,[\mathbf{K}(\mathbf{x})]=$ $\mathcal{G}([\mathbf{G}(\mathbf{x})]$ ), where $\mathcal{G}$ is independent of $\mathbf{x}$ (in fact, two types of representation are proposed), 
- a truncated reduced representation of random field $[\mathbf{G}]$,

- a parameterized representation for non-Gaussian random field $[\mathbf{K}]$,

- the parametric model-based representation $\mathbf{U}=\mathcal{B}(\boldsymbol{\Xi},[z])$.

Introduction a class of lower-bounded random fields for $[K]$ and normalization.

In order to normalize random field $[\mathbf{K}]$, a deterministic function $\mathbf{x} \mapsto[\underline{K}(\mathbf{x})]$ from $\Omega$ into $\mathbb{M}_{n}^{+}(\mathbb{R})$ is introduced such that, for all $\mathbf{x}$ in $\Omega$ and for all $\mathbf{z}$ in $\mathbb{R}^{n}$, $<[\underline{K}(\mathbf{x})] \mathbf{z}, \mathbf{z}>\geq \underline{k}_{0}\|\mathbf{z}\|^{2}$ and $<[\underline{K}(\mathbf{x})] \mathbf{z}, \mathbf{z}>\leq \underline{k}_{1}\|\mathbf{z}\|^{2}$ in which $\underline{k}_{0}$ and $\underline{k}_{1}$ are positive real constants, independent of $\mathbf{x}$, such that $0<\underline{k}_{0}<\underline{k}_{1}<+\infty$. These two technical inequalities correspond to the mathematical hypotheses that are required for obtaining a uniform deterministic elliptic operator whose coefficient is $[\underline{K}]$.

We introduce the following class of non-Gaussian positive-definite matrixvalued random fields $[\mathbf{K}]$, which admit a positive-definite matrix-valued lower bound, defined by

$$
[\mathbf{K}(\mathbf{x})]=\frac{1}{1+\varepsilon}[\underline{L}(\mathbf{x})]^{T}\left\{\varepsilon\left[I_{n}\right]+\left[\mathbf{K}_{0}(\mathbf{x})\right]\right\}[\underline{L}(\mathbf{x})] \quad, \quad \forall \mathbf{x} \in \Omega,
$$

in which $\varepsilon>0$ is any fixed positive real number, where $[\underline{L}(\mathbf{x})]$ is the upper triangular $(n \times n)$ real matrix such that $[\underline{K}(\mathbf{x})]=[\underline{L}(\mathbf{x})]^{T}[\underline{L}(\mathbf{x})]$, and where $\left[\mathbf{K}_{0}\right]=\left\{\left[\mathbf{K}_{0}(\mathbf{x})\right], \mathbf{x} \in \Omega\right\}$ is any random field indexed by $\Omega$, with values in $\mathbb{M}_{n}^{+}(\mathbb{R})$. Equation (11) can be inverted,

$$
\left[\mathbf{K}_{0}(\mathbf{x})\right]=(1+\varepsilon)[\underline{L}(\mathbf{x})]^{-T}[\mathbf{K}(\mathbf{x})][\underline{L}(\mathbf{x})]^{-1}-\varepsilon\left[I_{n}\right] \quad, \quad \forall \mathbf{x} \in \Omega .
$$

We have the following important properties for the class defined.

- Random field $[\mathbf{K}]$ is effectively with values in $\mathbb{M}_{n}^{+}(\mathbb{R})$. For all $\mathbf{x}$ fixed in $\Omega$, the lower bound is the matrix belonging to $\mathbb{M}_{n}^{+}(\mathbb{R})$ defined by $\left[K_{\varepsilon}(\mathbf{x})\right]=$ $\frac{\varepsilon}{1+\varepsilon}[\underline{K}(\mathbf{x})]$, and for all random matrix $\left[\mathbf{K}_{0}(\mathbf{x})\right]$ with values in $\mathbb{M}_{n}^{+}(\mathbb{R}),[\mathbf{K}(\mathbf{x})]$ defined by Eq. (12), is a random matrix with values in a subset of $\mathbb{M}_{n}^{+}(\mathbb{R})$ such that $[\mathbf{K}(\mathbf{x})] \geq\left[K_{\varepsilon}(\mathbf{x})\right]$ almost surely.

- For all integer $p \geq 1,\left\{[\mathbf{K}(\mathbf{x})]^{-1}, \mathbf{x} \in \Omega\right\}$ is a $p$-order random field with values in $\mathbb{M}_{n}^{+}(\mathbb{R})$, i.e., for all $\mathbf{x}$ in $\Omega, E\left\{\left\|[\mathbf{K}(\mathbf{x})]^{-1}\right\|_{F}^{p}\right\}<+\infty$ and, in particular, is a second-order random field.

- If $\left[\mathbf{K}_{0}\right]$ is a second-order random field, i.e., for all $\mathbf{x}$ in $\Omega, E\left\{\left\|\mathbf{K}_{0}(\mathbf{x})\right\|_{F}^{2}\right\}<$ $+\infty$, then $[\mathbf{K}]$ is a second-order random field, i.e., for all $\mathbf{x}$ in $\Omega, E\left\{\|\mathbf{K}(\mathbf{x})\|_{F}^{2}\right\}<$ $+\infty$. 
- If function $[\underline{K}]$ is chosen as the mean function of random field $[\mathbf{K}]$, i.e. $[\underline{K}(\mathbf{x})]=E\{[\mathbf{K}(\mathbf{x})]\}$ for all $\mathbf{x}$, then $E\left\{\left[\mathbf{K}_{0}(\mathbf{x})\right]\right\}$ is equal to $\left[I_{n}\right]$, what shows that random field $\left[\mathbf{K}_{0}\right]$ is normalized.

- The class of random fields defined by Eq. (11) yields a uniform stochastic elliptic operator $\mathcal{D}_{\mathbf{x}}$ that allows for studying the existence and uniqueness of a second-order random solution of a stochastic boundary value problem involving $\mathcal{D}_{\mathbf{x}}$.

\section{Construction of the nonlinear transformation $\mathcal{G}$.}

Two types of representation of random field $\left[\mathbf{K}_{0}\right]$ is proposed hereinafter: An "exponential-type representation" and a "square-type representation".

- Exponential-type representation of random field $\left[\mathbf{K}_{0}\right]$.

For all second-order random field $[\mathbf{G}]=\{[\mathbf{G}(\mathbf{x})], \mathbf{x} \in \Omega\}$ with values in $\mathbb{M}_{n}^{S}(\mathbb{R})$, which is not assumed to be Gaussian, the random field $\left[\mathbf{K}_{0}\right]$ defined by

$$
\left[\mathbf{K}_{0}(\mathbf{x})\right]=\exp _{\mathbb{M}}([\mathbf{G}(\mathbf{x})]) \quad, \quad \forall \mathbf{x} \in \Omega
$$

in which $\exp _{\mathbb{M}}$ denotes the exponential of symmetric square real matrices, is a random field with values in $\mathbb{M}_{n}^{+}(\mathbb{R})$. If $\left[\mathbf{K}_{0}\right]$ is any random field with values in $\mathbb{M}_{n}^{+}(\mathbb{R})$, then there exists a unique random field $[\mathbf{G}]$ with values in $\mathbb{M}_{n}^{S}(\mathbb{R})$ such that

$$
[\mathbf{G}(\mathbf{x})]=\log _{\mathbb{M}}\left(\left[\mathbf{K}_{0}(\mathbf{x})\right]\right) \quad, \quad \forall \mathbf{x} \in \Omega
$$

in which $\log _{\mathbb{M}}$ is the reciprocity mapping of $\exp _{\mathbb{M}}$, which is defined on $\mathbb{M}_{n}^{+}(\mathbb{R})$ with values in $\mathbb{M}_{n}^{S}(\mathbb{R})$, but in general, random field $[\mathbf{G}]$ is not a second-order random field. If $[\mathbf{G}]$ is any second-order random field with values in $\mathbb{M}_{n}^{+}(\mathbb{R})$, in general, the random field $\left[\mathbf{K}_{0}\right]=\exp _{\mathbb{M}}([\mathbf{G}])$ is not a second-order random field. Nevertheless, it can be proved that, if $\left[\mathbf{K}_{0}\right]$ and $\left[\mathbf{K}_{0}\right]^{-1}$ are second-order random fields with values in $\mathbb{M}_{n}^{+}(\mathbb{R})$, then there exists a second-order random field $[\mathbf{G}]$ with values in $\mathbb{M}_{n}^{S}(\mathbb{R})$ such that $\left[\mathbf{K}_{0}\right]=\exp _{\mathbb{M}}([\mathbf{G}])$.

- Square-type representation of random field $\left[\mathbf{K}_{0}\right]$.

Let $g \mapsto h(g ; a)$ be a given function from $\mathbb{R}$ in $\mathbb{R}^{+}$, depending on one positive real parameter $a$. For all fixed $a$, it is assumed that:

(i) $h(. ; a)$ is a strictly monotonically increasing function on $\mathbb{R}$, which means that $h(g ; a)<$

$$
h\left(g^{\prime} ; a\right) \text { if }-\infty<g<g^{\prime}<+\infty
$$


(ii) there are real numbers $0<c_{h}<+\infty$ and $0<c_{a}<+\infty$, such that, for all $g$ in $\mathbb{R}$, we

$$
\text { have } h(g ; a) \leq c_{a}+c_{h} g^{2} \text {. }
$$

The introduced hypotheses imply that, for all $a>0, g \mapsto h(g ; a)$ is a one-toone mapping from $\mathbb{R}$ onto $\mathbb{R}^{+}$and consequently, the reciprocity mapping, $v \mapsto$ $h^{-1}(v ; a)$, is a strictly monotonically increasing function from $\mathbb{R}^{+}$onto $\mathbb{R}$. The square type representation of random field $\left[\mathbf{K}_{0}\right]$, indexed by $\Omega$, with values in $\mathbb{M}_{n}^{+}(\mathbb{R})$, is defined by

$$
\left[\mathbf{K}_{0}(\mathbf{x})\right]=\mathbb{L}([\mathbf{G}(\mathbf{x})]) \quad, \quad \forall \mathbf{x} \in \Omega,
$$

in which $[\mathbf{G}]=\{[\mathbf{G}(\mathbf{x})], \mathbf{x} \in \Omega\}$ is a second-order random field with values in $\mathbb{M}_{n}^{S}(\mathbb{R})$ and where $[G] \mapsto \mathbb{L}([G])$ is a measurable mapping from $\mathbb{M}_{n}^{S}(\mathbb{R})$ into $\mathbb{M}_{n}^{+}(\mathbb{R})$ which is defined as follows. The matrix $\left[K_{0}\right]=\mathbb{L}([G]) \in \mathbb{M}_{n}^{+}(\mathbb{R})$ is written as $\left[K_{0}\right]=[L]^{T}[L]$ in which $[L]$ belongs to $\mathbb{M}_{n}^{U}(\mathbb{R})$, which is written as $[L]=\mathcal{L}([G])$ where $[G] \mapsto \mathcal{L}([G])$ is the measurable mapping from $\mathbb{M}_{n}^{S}(\mathbb{R})$ into $\mathbb{M}_{n}^{U}(\mathbb{R})$ defined by

$$
[\mathcal{L}([G])]_{j k}=[G]_{j k}, 1 \leq j<k \leq n,[\mathcal{L}([G])]_{j j}=\sqrt{h\left([G]_{j j} ; a_{j}\right)}, 1 \leq j \leq n,
$$

in which $a_{1}, \ldots, a_{n}$ are positive real numbers. If $\left[\mathbf{K}_{0}\right]$ is any random field indexed by $\Omega$ with values in $\mathbb{M}_{n}^{+}(\mathbb{R})$, then there exists a unique random field $[\mathbf{G}]$ with values in $\mathbb{M}_{n}^{S}(\mathbb{R})$ such that

$$
[\mathbf{G}(\mathbf{x})]=\mathbb{L}^{-1}\left(\left[\mathbf{K}_{0}(\mathbf{x})\right]\right) \quad, \quad \forall \mathbf{x} \in \Omega,
$$

in which $\mathbb{L}^{-1}$ is the reciprocity function of $\mathbb{L}$, from $\mathbb{M}_{n}^{+}(\mathbb{R})$ into $\mathbb{M}_{n}^{S}(\mathbb{R})$, which is explicitly defined as follows. For all $1 \leq j \leq k \leq n$,

$$
[\mathbf{G}(\mathbf{x})]_{j k}=\left[\mathcal{L}^{-1}([\mathbf{L}(\mathbf{x})])\right]_{j k} \quad, \quad[\mathbf{G}(\mathbf{x})]_{k j}=[\mathbf{G}(\mathbf{x})]_{j k},
$$

in which $[L] \mapsto \mathcal{L}^{-1}([L])$ is the unique reciprocity mapping of $\mathcal{L}$ (due to the existence of $v \mapsto h^{-1}(v ; a)$ ) defined on $\mathbb{M}_{n}^{U}(\mathbb{R})$, and where $[\mathbf{L}(\mathbf{x})]$ follows from the Cholesky factorization of random matrix $\left[\mathbf{K}_{0}(\mathbf{x})\right]=[\mathbf{L}(\mathbf{x})]^{T}[\mathbf{L}(\mathbf{x})]$ (see Eq. (15)). Example of function $h$. An example of such a function is given in "An algebraic prior stochastic model $\left[\boldsymbol{K}^{\mathrm{APS}}\right]$ for the case of anisotropic statistical fluctuations" of the present Section. Nevertheless, for the sake of clarity, we detail it hereinafter. Let $h=h^{\mathrm{APSM}}$ be the function $h^{\mathrm{APSM}}$ defined in [58] as follows. Let be $s=\delta / \sqrt{n+1}$ in which $\delta$ is a parameter such that $0<\delta<$ 
$\sqrt{(n+1) /(n-1)}$ and which allows the statistical fluctuations level to be controlled. Let be $a_{j}=1 /\left(2 s^{2}\right)+(1-j) / 2>0$ and $h^{\mathrm{APSM}}(g ; a)=2 s^{2} F_{\Gamma_{a}}^{-1}\left(F_{W}(g / s)\right)$ with $F_{W}(\widetilde{w})=\int_{-\infty}^{\widetilde{w}} \frac{1}{\sqrt{2 \pi}} \exp \left(-\frac{1}{2} w^{2}\right) d w$ and $F_{\Gamma_{a}}^{-1}(u)=\gamma$ the reciprocal function such that $F_{\Gamma_{a}}(\gamma)=u$ with $F_{\Gamma_{a}}(\gamma)=\int_{0}^{\gamma} \frac{1}{\Gamma(a)} t^{a-1} e^{-t} d t$ and $\Gamma(a)=\int_{0}^{+\infty} t^{a-1} e^{-t}$ $d t$. Then, for all $j=1, \ldots, n$, it can be proved that $g \mapsto h^{\mathrm{APSM}}\left(g ; a_{j}\right)$ is a strictly monotonically increasing function from $\mathbb{R}$ into $\mathbb{R}^{+}$and there are positive real numbers $c_{h}$ and $c_{a_{j}}$ such that, for all $g$ in $\mathbb{R}$, we have $h^{\mathrm{APSM}}\left(g ; a_{j}\right) \leq c_{a_{j}}+c_{h} g^{2}$. In addition, it can easily be seen that the reciprocity function is written as $h^{\mathrm{APSM}^{-1}}(v ; a)=$ $s F_{W}^{-1}\left(F_{\Gamma_{a}}\left(v /\left(2 s^{2}\right)\right)\right.$.

- Construction of the transformation $\mathcal{G}$ and its inverse $\mathcal{G}^{-1}$.

For the exponential-type representation, the transformation $\mathcal{G}$ is defined by Eq. (11) with Eq. (13), and its inverse $\mathcal{G}^{-1}$ is defined by Eq. (14) with Eq. (12), and are such that, for all $\mathbf{x}$ in $\Omega$,

$$
\begin{gathered}
{[\mathbf{K}(\mathbf{x})]=\mathcal{G}([\mathbf{G}(\mathbf{x})]):=\frac{1}{1+\varepsilon}[\underline{L}(\mathbf{x})]^{T}\left\{\varepsilon\left[I_{n}\right]+\exp _{\mathbb{M}}([\mathbf{G}(\mathbf{x})])\right\}[\underline{L}(\mathbf{x})],} \\
{[\mathbf{G}(\mathbf{x})]=\mathcal{G}^{-1}([\mathbf{K}(\mathbf{x})]):=\log _{\mathbb{M}}\left\{(1+\varepsilon)[\underline{L}(\mathbf{x})]^{-T}[\mathbf{K}(\mathbf{x})][\underline{L}(\mathbf{x})]^{-1}-\varepsilon\left[I_{n}\right]\right\} .}
\end{gathered}
$$

For the square-type representation, the transformation $\mathcal{G}$ is defined by Eq. (11) with Eq. (15), and its inverse $\mathcal{G}^{-1}$ is defined by Eq. (17) with Eq. (12), and are such that, for all $\mathbf{x}$ in $\Omega$,

$$
\begin{gathered}
{[\mathbf{K}(\mathbf{x})]=\mathcal{G}([\mathbf{G}(\mathbf{x})]):=\frac{1}{1+\varepsilon}[\underline{L}(\mathbf{x})]^{T}\left\{\varepsilon\left[I_{n}\right]+[\mathcal{L}([\mathbf{G}(\mathbf{x})])]^{T}[\mathcal{L}([\mathbf{G}(\mathbf{x})])]\right\}[\underline{L}(\mathbf{x})],} \\
{[\mathbf{G}(\mathbf{x})]=\mathcal{G}^{-1}([\mathbf{K}(\mathbf{x})]):=\mathbb{L}^{-1}\left\{(1+\varepsilon)[\underline{L}(\mathbf{x})]^{-T}[\mathbf{K}(\mathbf{x})][\underline{L}(\mathbf{x})]^{-1}-\varepsilon\left[I_{n}\right]\right\} .}
\end{gathered}
$$

Let $\mathbb{M}_{n}^{+b}(\mathbb{R})$ be the subset of $\mathbb{M}_{n}^{+}(\mathbb{R})$, constituted of all the positive-definite matrices $[K]$ such that, for all $\mathbf{x}$ in $\Omega$, the matrix $[K]-\left[K_{\varepsilon}(\mathbf{x})\right]>0$. Transformation $\mathcal{G}$ maps $\mathbb{M}_{n}^{S}(\mathbb{R})$ into $\mathbb{M}_{n}^{+b}(\mathbb{R}) \subset \mathbb{M}_{n}^{+}(\mathbb{R})$, and $\mathcal{G}^{-1}$ maps $\mathbb{M}_{n}^{+b}(\mathbb{R})$ into $\mathbb{M}_{n}^{S}(\mathbb{R})$.

\section{Truncated reduced representation of second-order random field $[G]$ and its polynomial chaos expansion}

Two versions of the nonlinear transformation $\mathcal{G}$ from $\mathbb{M}_{n}^{S}(\mathbb{R})$ into $\mathbb{M}_{n}^{+}(\mathbb{R})$ are defined by Eqs. (19) and (21). For the statistical inverse problem, $[\mathbf{G}]$ is chosen in the class of the second-order random field indexed by $\Omega$ with values in $\mathbb{M}_{n}^{S}(\mathbb{R})$, is reduced using its truncated Karhunen-Loève decomposition in which the random 
coordinates are represented using a truncated polynomial Gaussian chaos. Consequently, the approximation $\left[\mathbf{G}^{\left(m, N, N_{g}\right)}\right]$ of the non-Gaussian second-order random field $[\mathbf{G}]$ is introduced such that

$$
\begin{gathered}
{\left[\mathbf{G}^{\left(m, N, N_{g}\right)}(\mathbf{x})\right]=\left[G_{0}(\mathbf{x})\right]+\sum_{i=1}^{m} \sqrt{\lambda_{i}}\left[G_{i}(\mathbf{x})\right] \eta_{i},} \\
\eta_{i}=\sum_{j=1}^{N} y_{i}^{j} \Psi_{j}(\boldsymbol{\Xi})
\end{gathered}
$$

in which

- $\lambda_{1} \geq \ldots \geq \lambda_{m}>0$ are the dominant eigenvalues and $\left[G_{1}\right], \ldots,\left[G_{m}\right]$ are the corresponding orthonormal eigenfunctions of the covariance operator $\operatorname{Cov}_{\mathbf{G}}$ of random field $[\mathbf{G}]$. The kernel of this covariance operator is the tensor-valued crosscovariance function $C_{\mathbf{G}}\left(\mathbf{x}, \mathbf{x}^{\prime}\right)$ of $[\mathbf{G}]$, which is assumed to be square integrable on $\Omega \times \Omega$

- $\left\{\Psi_{j}\right\}_{j=1}^{N}$ only depends on a random vector $\Xi=\left(\Xi_{1}, \ldots, \Xi_{N_{g}}\right)$ of $N_{g} \leq$ $m$ independent normalized Gaussian random variables $\Xi_{1}, \ldots, \Xi_{N_{g}}$ defined on probability space $(\Theta, \mathcal{T}, \mathcal{P})$,

- $\left\{\Psi_{j}\right\}_{j=1}^{N}$ are the polynomial Gaussian chaos that are written as $\Psi_{j}(\boldsymbol{\Xi})=$ $\Phi_{\alpha_{1}}\left(\Xi_{1}\right) \times \ldots \times \Phi_{\alpha_{N_{g}}}\left(\Xi_{N_{g}}\right)$, in which $j$ is the index associated with the multi-index $\boldsymbol{\alpha}=\left(\alpha_{1}, \ldots, \alpha_{N_{g}}\right)$ in $\mathbb{N}^{N_{g}}$, the degree of $\Psi_{j}(\boldsymbol{\Xi})$ is $\alpha_{1}+\ldots+\alpha_{N_{g}} \leq N_{d}$ and where $\Phi_{\alpha_{k}}\left(\Xi_{k}\right)$ is the normalized univariate Hermite polynomial on $\mathbb{R}$. Consequently, $\left\{\Psi_{j}\right\}_{j=1}^{N}$ are composed of the normalized multivariate Hermite polynomials such that $E\left\{\Psi_{j}(\boldsymbol{\Xi}) \Psi_{j^{\prime}}(\boldsymbol{\Xi})\right\}=\delta_{j j^{\prime}}$,

- the constant Hermite polynomial $\Psi_{0}(\boldsymbol{\Xi})=1$ with index $j=0$ (corresponding to the zero multi-index $(0, \ldots, 0))$ is not included in Eq. (24). Consequently, the integer $N$ is such that $N=\left(N_{d}+N_{g}\right) ! /\left(N_{d} ! N_{g} !\right)-1$ where $N_{d}$ is the maximum degree of the normalized multivariate Hermite polynomials,

- $y_{i}^{j}$ are the coefficients that are supposed to verify $\sum_{j=1}^{N} y_{i}^{j} y_{i^{\prime}}^{j}=\delta_{i i^{\prime}}$, which ensures that the random variables, $\left\{\eta_{i}\right\}_{i=1}^{m}$, are uncorrelated centered random variables with unit variance, which means that $E\left\{\eta_{i} \eta_{i^{\prime}}\right\}=\delta_{i i^{\prime}}$. The relation between the coefficients can be rewritten as

$$
[z]^{T}[z]=\left[I_{m}\right],
$$

in which $[z] \in \mathbb{M}_{N, m}(\mathbb{R})$ is such that

$$
[z]_{j i}=y_{i}^{j} \quad, \quad 1 \leq i \leq m \quad, \quad 1 \leq j \leq N .
$$


Introducing the random vectors $\boldsymbol{\eta}=\left(\eta_{1}, \ldots, \eta_{m}\right)$ and $\boldsymbol{\Psi}(\boldsymbol{\Xi})=\left(\Psi_{1}(\boldsymbol{\Xi}), \ldots\right.$, $\Psi_{N}(\boldsymbol{\Xi})$ ), Eq. (24) can be rewritten as

$$
\boldsymbol{\eta}=[z]^{T} \boldsymbol{\Psi}(\boldsymbol{\Xi})
$$

Equation (25) means that $[z]$ belongs to the compact Stiefel manifold

$$
\mathbb{V}_{m}\left(\mathbb{R}^{N}\right)=\left\{[z] \in \mathbb{M}_{N, m}(\mathbb{R}) ;[z]^{T}[z]=\left[I_{m}\right]\right\} .
$$

\section{Parameterization of compact Stiefel manifold $\mathbb{V}_{m}\left(\mathbb{R}^{N}\right)$}

A parametrization of $\mathbb{V}_{m}\left(\mathbb{R}^{N}\right)$ defined by Eq. (28) is given hereinafter. For all $\left[z_{0}\right]$ fixed in $\mathbb{V}_{m}\left(\mathbb{R}^{N}\right)$, let $T_{\left[z_{0}\right]}$ be the tangent vector space to $\mathbb{V}_{m}\left(\mathbb{R}^{N}\right)$ at $\left[z_{0}\right]$. The objective is to construct a mapping $[w] \mapsto[z]=\mathcal{R}_{\left[z_{0}\right]}([w])$ from $T_{\left[z_{0}\right]}$ onto $\mathbb{V}_{m}\left(\mathbb{R}^{N}\right)$ such that $\mathcal{R}_{\left[z_{0}\right]}([0])=\left[z_{0}\right]$, and such that, if $[w]$ belongs to a subset of $T_{\left[z_{0}\right]}$, this subset being centered in $[w]=[0]$ and having a sufficiently small diameter, then $[z]=\mathcal{R}_{\left[z_{0}\right]}([w])$ belongs to a subset of $\mathbb{V}_{m}\left(\mathbb{R}^{N}\right)$, approximatively centered in $[z]=\left[z_{0}\right]$. There are several possibilities for constructing such a parameterization (see for instance $[19 ; 1]$ ). For instance, a parameterization can be constructed as described in [48] using the geometry of algorithms with orthogonality constraints [19]. Hereinafter, we present the construction proposed in [1] for which the algorithm has a small complexity with respect to the other possible possibilities. Let us assume that $N>m$ that is generally the case. For $\left[z_{0}\right]$ fixed in $\mathbb{V}_{m}\left(\mathbb{R}^{N}\right)$, the mapping $\mathcal{R}_{\left[z_{0}\right]}$ is defined by

$$
\left.[z]=\mathcal{R}_{\left[z_{0}\right]}([w]):=\operatorname{qr}\left(\left[z_{0}\right]+\sigma[w]\right]\right) \quad, \quad[w] \in T_{\left[z_{0}\right]},
$$

in which qr is the mapping that corresponds to the QR economy-size decomposition of matrix $\left[z_{0}\right]+\sigma[w]$, for which only the first $m$ columns of matrix $[q]$ such that $\left[z_{0}\right]+\sigma[w]=[q][r]$ are computed and such that $[z]^{T}[z]=\left[I_{m}\right]$. In Eq. (29), $\sigma$ allows the diameter of the subset of $T_{\left[z_{0}\right]}$ centered in $[0]$ to be controlled.

\section{Parameterized representation for non-gaussian random field $[\mathbf{K}]$}

Let $\left\{\left[\mathbf{G}^{\left(m, N, N_{g}\right)}(\mathbf{x})\right], \mathbf{x} \in \Omega\right\}$ be defined by Eqs. (23) and (24), and let $\mathcal{G}$ be defined by Eq. (19) for the exponential-type representation, and by Eq. (21) for the square-type representation. The corresponding parameterized representation for non-Gaussian positive-definite matrix-valued random field $\{[\mathbf{K}(\mathbf{x})], \mathbf{x} \in \Omega\}$ is denoted by $\left\{\left[\mathbf{K}^{\left(m, N, N_{g}\right)}(\mathbf{x})\right], \mathbf{x} \in \Omega\right\}$ and is rewritten, for all $\mathbf{x}$ in $\Omega$, as

$$
\left[\mathbf{K}^{\left(m, N, N_{g}\right)}(\mathbf{x})\right]=\mathcal{K}^{\left(m, N, N_{g}\right)}(\mathbf{x}, \boldsymbol{\Xi},[z]),
$$


in which $(\mathbf{x}, \boldsymbol{\xi},[z]) \mapsto \mathcal{K}^{\left(m, N, N_{g}\right)}(\mathbf{x}, \boldsymbol{\xi},[z])$ is a deterministic mapping defined on $\Omega \times \mathbb{R}^{N_{g}} \times \mathbb{V}_{m}\left(\mathbb{R}^{N}\right)$ with values in $\mathbb{M}_{n}^{+}(\mathbb{R})$ such that

$$
\mathcal{K}^{\left(m, N, N_{g}\right)}(\mathbf{x}, \boldsymbol{\xi},[z])=\mathcal{G}\left(\left[G_{0}(\mathbf{x})\right]+\sum_{i=1}^{m} \sqrt{\lambda_{i}}\left[G_{i}(\mathbf{x})\right]\left\{[z]^{T} \mathbf{\Psi}(\boldsymbol{\xi})\right\}_{i}\right) .
$$

Parametric model-based representation of random observation model $\mathbf{U}$

From Eqs. (9) and (30), the parametric model-based representation of random model observation $\mathbf{U}$ with values in $\mathbb{R}^{N_{U}}$, corresponding to the representation $\left\{\left[\mathbf{K}^{\left(m, N, N_{g}\right)}(\mathbf{x})\right], \mathbf{x} \in \Omega\right\}$ of random field $\{[\mathbf{K}(\mathbf{x})], \mathbf{x} \in \Omega\}$, is denoted by $\mathbf{U}^{\left(m, N, N_{g}\right)}$ and is written as

$$
\mathbf{U}^{\left(m, N, N_{g}\right)}=\boldsymbol{B}^{\left(m, N, N_{g}\right)}(\boldsymbol{\Xi},[z]),
$$

in which $(\boldsymbol{\xi},[z]) \mapsto \boldsymbol{B}^{\left(m, N, N_{g}\right)}(\boldsymbol{\xi},[z])$ is a deterministic mapping defined on $\mathbb{R}^{N_{g}} \times$ $\mathbb{V}_{m}\left(\mathbb{R}^{N}\right)$ with values in $\mathbb{R}^{N_{U}}$ such that

$$
\mathcal{B}^{\left(m, N, N_{g}\right)}(\boldsymbol{\xi},[z])=\mathbf{h}\left(\mathcal{K}^{\left(m, N, N_{g}\right)}\left(\mathbf{x}^{1}, \boldsymbol{\xi},[z]\right), \ldots, \mathcal{K}^{\left(m, N, N_{g}\right)}\left(\mathbf{x}^{N_{p}}, \boldsymbol{\xi},[z]\right)\right) .
$$

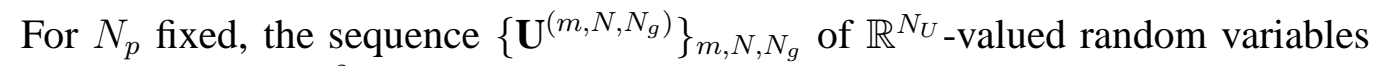
converge to $\mathbf{U}$ in $\mathcal{L}_{N_{U}}^{2}$.

\section{Methodology for Solving the Statistical Inverse Problem in High Stochas- tic Dimension}

A general methodology is presented for solving the statistical inverse problem defined in the previous section entitled "Setting the Statistical Inverse Problem to Be Solved in High Stochastic Dimension". The steps of the identification procedure are defined hereinafter.

Step 1: Introduction of a family $\left\{\left[\mathrm{K}^{\mathrm{APSM}}(\mathbf{x} ; \mathbf{s})\right], \mathbf{x} \in \Omega\right\}$ of algebraic prior stochastic models (APSM) for non-Gaussian random field $[\mathbf{K}]$.

The first step consists in introducing a family $\left\{\left[\mathbf{K}^{\mathrm{APSM}}(\mathbf{x} ; \mathbf{s})\right], \mathbf{x} \in \Omega\right\}$ of algebraic prior stochastic models (APSM) for the non-Gaussian second-order random field $[\mathbf{K}]$, defined on $(\Theta, \mathcal{T}, \mathcal{P})$, indexed by $\Omega$, with values in $\mathbb{M}_{n}^{+}(\mathbb{R})$, which has been introduced in the previous paragraph entitled "Stochastic elliptic operator and boundary value problem". This family depends on an unknown hyperparameter $\mathbf{s}$ belonging to an admissible set $\mathcal{C}_{s}$ that is a subset of $\mathbb{R}^{N_{s}}$, for which the dimension, $N_{s}$, is assumed to be relatively small, while the stochastic dimension of $\left[\mathbf{K}^{\mathrm{APSM}}\right]$ is high. For instance, $\mathbf{s}$ can be made up of the mean function, a matrix-valued lower 
bound, some spatial-correlation lengths, some parameters controlling the statistical fluctuations and the shape of the tensor-valued correlation function. For $\mathbf{s}$ fixed in $\mathcal{C}_{s}$, the probability distribution (i.e. the system of marginal probability distributions) of random field $\left[\mathbf{K}^{\mathrm{APSM}}\right]$ and the corresponding generator of independent realizations are assumed to have been constructed and consequently, are assumed to be known.

An example of such a construction is explicitly given in the next section entitled "Construction of a Family of Algebraic Prior Stochastic Models".

As it has been explained in the previous paragraph entitled "Algebraic prior stochastic models of the model parameters of BVP" of Section "Brief History", Step 1 is a fundamental step of the methodology. The real capability to correctly solve the statistical inverse problem in high stochastic dimension is directly related to the pertinence and to the quality of the constructed APSM that allows for enriching the information in order to overcome the lack of experimental data (only partial experimental data are assumed to be available). Such a construction must be carried out using the MaxEnt principle of Information Theory, under the constraints defined by the available information such as the symmetries, the positiveness, the invertibility in mean square, the boundedness, the capability of the APSM to exhibit simultaneously anisotropic statistical fluctuations and some statistical fluctuations in a given symmetry class such as isotropic, cubic, transversely isotropic, orthotropic, etc. In addition, the corresponding generators of realizations must be developed. For the MaxEnt principle and the construction of generators, we refer the reader to Section "Random Matrix Models and nonparametric Method for Uncertainty Quantification" in part II of the present Handbook on Uncertainty Quantification.

\section{Step 2: Identification of an optimal algebraic prior stochastic model (OAPSM)} for non-Gaussian random field $[\mathbf{K}]$.

The second step consists in identifying an optimal value $\mathbf{s}^{\text {opt }}$ in $\mathcal{C}_{s}$ of hyperparameter $\mathbf{s}$ using experimental data sets $\mathbf{u}^{\exp , 1}, \ldots, \mathbf{u}^{\exp , \nu_{\exp }}$ relative to the random model observation $\mathbf{U}$ of the stochastic computational model, which is written, taking into account Eq. (9), as

$$
\mathbf{U}=\mathbf{h}\left(\left[\mathbf{K}^{\mathrm{APSM}}\left(\mathbf{x}^{1} ; \mathbf{s}\right)\right], \ldots,\left[\mathbf{K}^{\mathrm{APSM}}\left(\mathbf{x}^{N_{p}} ; \mathbf{s}\right)\right]\right) .
$$

The calculation of $\mathbf{s}^{\text {opt }}$ in $\mathcal{C}_{s}$ can be carried out by using the maximum likelihood method:

$$
\mathbf{s}^{\text {opt }}=\arg \max _{\mathbf{s} \in \mathcal{C}_{s}} \sum_{\ell=1}^{\nu_{\text {exp }}} \log p_{\mathbf{U}}\left(\mathbf{u}^{\exp , \ell} ; \mathbf{s}\right),
$$


in which $p_{\mathbf{U}}\left(\mathbf{u}^{\exp , \ell} ; \mathbf{s}\right)$ is the value, in $\mathbf{u}=\mathbf{u}^{\exp , \ell}$, of the probability density function $p_{\mathbf{U}}(\mathbf{u} ; \mathbf{s})$ of the random vector $\mathbf{U}$ defined by Eq. (34) and depending on $\mathbf{s}$. The optimal algebraic prior model $\left\{\left[\mathbf{K}^{\mathrm{OAPSM}}(\mathbf{x})\right], \mathbf{x} \in \Omega\right\}:=\left\{\left[\mathbf{K}^{\mathrm{APSM}}\left(\mathbf{x} ; \mathbf{s}^{\text {opt }}\right)\right], \mathbf{x} \in \Omega\right\}$ is then obtained. Using the generator of realizations of the optimal APSM, $\nu_{\mathrm{KL}}$ independent realizations $\left[K^{(1)}\right], \ldots,\left[K^{\left(\nu_{\mathrm{KL}}\right)}\right]$ can be computed such that, for $\ell=$ $1, \ldots, \nu_{\mathrm{KL}}$ and $\theta_{\ell} \in \Theta$, the deterministic field $\left[K^{(\ell)}\right]:=\left\{\left[K^{(\ell]}(\mathbf{x})\right], \mathbf{x} \in \Omega\right\}$ is such that

$$
\left[K^{(\ell)}\right]=\left\{\left[\mathbf{K}^{\mathrm{OAPSM}}\left(\mathbf{x} ; \theta_{\ell}\right)\right], \mathbf{x} \in \Omega\right\} .
$$

These realizations can be generated at points $\mathbf{x}^{1}, \ldots, \mathbf{x}^{N_{p}}$ (or at any other points), with $\nu_{\mathrm{KL}}$ as large as it is desired without inducing a significant computational cost.

Step 3: Choice of an adapted representation for non-Gaussian random field $[K]$ and optimal algebraic prior stochastic model for non-Gaussian random field $[\mathbf{G}]$.

For a fixed choice of the type of representation for random field $[\mathbf{K}]$ given by Eq. (19) (exponential type) or (21) (square type), the corresponding optimal algebraic prior model $\left\{\left[\mathbf{G}^{\mathrm{OAPSM}}(\mathbf{x})\right], \mathbf{x} \in \Omega\right\}$ of random field $\{[\mathbf{G}(\mathbf{x})], \mathbf{x} \in \Omega\}$ is written as

$$
\left[\mathbf{G}^{\mathrm{OAPSM}}(\mathbf{x})\right]=\mathcal{G}^{-1}\left(\left[\mathbf{K}^{\mathrm{OAPSM}}(\mathbf{x})\right]\right) \quad, \quad \forall \mathbf{x} \in \Omega,
$$

in which $\mathcal{G}^{-1}$ is defined by Eq. (20) (exponential type) or by Eq. (22) (square type). It is assumed that random field $\left[\mathbf{G}^{\mathrm{OAPS}}\right]$ is a second-order random field. From the $\nu_{\mathrm{KL}}$ independent realizations $\left[K^{(1)}\right], \ldots,\left[K^{\left(\nu_{\mathrm{KL}}\right)}\right]$ of random field $\left[\mathbf{K}^{\mathrm{OAPSM}}\right]$ (see Eq. (36)), it can be deduced the $\nu_{\mathrm{KL}}$ independent realizations $\left[G^{(1)}\right], \ldots,\left[G^{\left(\nu_{\mathrm{KL}}\right)}\right]$ of random field $\left[\mathbf{G}^{\mathrm{OAPSM}}\right]$ such that,

$$
\left[G^{(\ell)}(\mathbf{x})\right]=\mathcal{G}^{-1}\left(\left[K^{(\ell)}(\mathbf{x})\right]\right) \quad, \quad \forall \mathbf{x} \in \Omega \quad, \quad \ell=1, \ldots, \nu_{\mathrm{KL}} .
$$

\section{Step 4: Construction of a truncated reduced representation of second-order random field $\left[\mathbf{G}^{\mathrm{OAPSM}}\right]$. \\ The $\nu_{\mathrm{KL}}$ independent realizations $\left[G^{(1)}\right], \ldots,\left[G^{\left(\nu_{\mathrm{KL}}\right)}\right]$ of random field $\left[\mathbf{G}^{\mathrm{OAPSM}}\right]$ (com- puted with Eq. (38)) are used to calculate, for random field $\left[\mathbf{G}^{\mathrm{OAPS}}\right]$, an estimation, $\left[G_{0}\right]$, of the mean function and an estimation, $\operatorname{Cov}_{\mathbf{G}^{\text {OAPSM }}}$, of the covariance operator whose kernel is the tensor-valued cross-covariance function $C_{\mathbf{G}^{\text {OAPsM }}}\left(\mathbf{x}, \mathbf{x}^{\prime}\right)$ that is assumed to be square integrable on $\Omega \times \Omega$. The first $m$ eigenvalues $\lambda_{1} \geq \ldots \geq$ $\lambda_{m}$ and the corresponding orthonormal eigenfunctions $\left[G_{1}\right], \ldots,\left[G_{m}\right]$ of covari- ance operator $\operatorname{Cov}_{\mathbf{G}^{\text {OAPSM }}}$ are then computed. For a given convergence tolerance,}


the optimal value of $m$ is calculated, and the truncated reduced representation $\left\{\left[\mathbf{G}^{\mathrm{OAPSM}(m)}(\mathbf{x})\right], \mathbf{x} \in \Omega\right\}$ of the second-order random field $\left\{\left[\mathbf{G}^{\mathrm{OAPSM}}(\mathbf{x})\right], \mathbf{x} \in \Omega\right\}$ is written (see Eq. (23)) as

$$
\left[\mathbf{G}^{\operatorname{OAPSM}(m)}(\mathbf{x})\right]=\left[G_{0}(\mathbf{x})\right]+\sum_{i=1}^{m} \sqrt{\lambda_{i}}\left[G_{i}(\mathbf{x})\right] \eta_{i}^{\text {OAPSM }} \quad, \quad \forall \mathbf{x} \in \Omega .
$$

Using the $\nu_{\mathrm{KL}}$ independent realizations $\left[G^{(1)}\right], \ldots,\left[G^{\left(\nu_{\mathrm{KL}}\right)}\right]$ of random field $\left[\mathbf{G}^{\mathrm{OAPSM}}\right]$ calculated with Eq. (38), $\nu_{\mathrm{KL}}$ independent realizations $\boldsymbol{\eta}^{(1)}, \ldots, \boldsymbol{\eta}^{\left(\nu_{\mathrm{KL}}\right)}$ of the random vector $\boldsymbol{\eta}^{\text {OAPSM }}=\left(\eta_{1}^{\text {OAPSM }}, \ldots, \eta_{m}^{\text {OAPSM }}\right)$ are calculated, for $i=1, \ldots, m$ and for $\ell=1, \ldots, \nu_{\mathrm{KL}}$, by

$$
\eta_{i}^{(\ell)}=\frac{1}{\sqrt{\lambda_{i}}} \int_{\Omega} \ll\left[G^{(\ell)}(\mathbf{x})\right]-\left[G_{0}(\mathbf{x})\right],\left[G_{i}(\mathbf{x}] \gg d \mathbf{x}\right.
$$

Step 5: Construction of a truncated polynomial chaos expansion of $\eta^{\text {OAPSM }}$ and representation of random field $\left[K^{\mathrm{OAPSM}}\right]$.

Using independent realizations $\boldsymbol{\eta}^{(1)}, \ldots, \boldsymbol{\eta}^{\left(\nu_{\mathrm{KL}}\right)}$ of random vector $\boldsymbol{\eta}^{\text {OAPSM }}$ (see Eq. (40)), this step consists in constructing the approximation $\boldsymbol{\eta}^{\text {chaos }}\left(N_{d}, N_{g}\right)=$ $\left(\eta_{1}^{\text {chaos }}\left(N_{d}, N_{g}\right), \ldots, \eta_{m}^{\text {chaos }}\left(N_{d}, N_{g}\right)\right)$ of $\boldsymbol{\eta}^{\text {OAPSM }}$ using Eq. (27), for which the matrix $[z]$ in $\mathbb{M}_{N, m}(\mathbb{R})$ of the coefficients verifies $[z]^{T}[z]=\left[I_{m}\right]$,

$$
\boldsymbol{\eta}^{\text {OAPSM }} \simeq \boldsymbol{\eta}^{\text {chaos }}\left(N_{d}, N_{g}\right) \quad, \quad \boldsymbol{\eta}^{\text {chaos }}\left(N_{d}, N_{g}\right)=[z]^{T} \boldsymbol{\Psi}(\boldsymbol{\Xi}),
$$

in which the integer $N$ is defined by

$$
N=\operatorname{h}\left(N_{d}, N_{g}\right):=\left(N_{d}+N_{g}\right) ! /\left(N_{d} ! N_{g} !\right)-1,
$$

where the integer $N_{d}$ is the maximum degree of the normalized multivariate Hermite polynomials and $N_{g}$ the dimension of random vector $\boldsymbol{\Xi}$. In Eq. (41)), the symbol " $\simeq$ " means that the mean-square convergence is reached for $N_{d}$ and $N_{g}$ (with $N_{g} \leq m$ ) sufficiently large.

- Identification of an optimal value $\left[z_{0}\left(N_{d}, N_{g}\right)\right]$ of $[z]$ for a fixed value of $N_{d}$ and $N_{g}$.

For a fixed value of $N_{d}$ and $N_{g}$ such that $N_{d} \geq 1$ and $1 \leq N_{g} \leq m$, the identification of $[z]$ is performed using the maximum likelihood method. The log-likelihood function is written as

$$
\mathcal{L}([z])=\sum_{\ell=1}^{\nu_{\mathrm{KL}}} \log p_{\boldsymbol{\eta}^{\text {chaos }}\left(N_{d}, N_{g}\right)}\left(\boldsymbol{\eta}^{(\ell)} ;[z]\right)
$$


and the optimal value $\left[z_{0}\left(N_{d}, N_{g}\right)\right]$ of $[z]$ is given by

$$
\left[z_{0}\left(N_{d}, N_{g}\right)\right]=\arg \max _{[z] \in \mathbb{V}_{m}\left(\mathbb{R}^{N}\right)} \mathcal{L}([z])
$$

in which $\mathbb{V}_{m}\left(\mathbb{R}^{N}\right)$ is defined by Eq. (28).

(i) For $[z]$ fixed in $\mathbb{V}_{m}\left(\mathbb{R}^{N}\right)$, the probability density function $\mathbf{e} \mapsto p_{\eta^{\text {chaos }}\left(N_{d}, N_{g}\right)}(\mathbf{e} ;[z])$ of random variable $\boldsymbol{\eta}^{\text {chaos }}\left(N_{d}, N_{g}\right)$ is estimated by the multidimensional kernel density estimation method using $\nu_{\text {chaos }}$ independent realizations $\boldsymbol{\eta}^{\text {chaos (1) }}, \ldots \ldots$, $\boldsymbol{\eta}^{\text {chaos }\left(\nu_{\text {chaos }}\right)}$ of random vector $\boldsymbol{\eta}^{\text {chaos }}\left(N_{d}, N_{g}\right)$, which are such that $\boldsymbol{\eta}^{\text {chaos }(\ell)}=$ $[z]^{T} \boldsymbol{\Psi}\left(\boldsymbol{\Xi}^{(\ell)}\right)$ in which $\boldsymbol{\Xi}^{(1)}, \ldots, \boldsymbol{\Xi}^{\left(\nu_{\text {chaos }}\right)}$ are $\nu_{\text {chaos }}$ independent realizations of $\boldsymbol{\Xi}$.

(ii) For the high-dimension case, i.e. for $m \times N$ very large, the optimization problem defined by Eq. (44) must be solved with adapted and robust algorithms:

- The first one is required for generating the independent realizations $\Psi_{j}\left(\boldsymbol{\Xi}^{(\ell)}\right)$ of $\Psi_{j}(\boldsymbol{\Xi})$ in preserving the orthogonality condition for any high values of $N_{g}$ and $N_{d}$. An efficient algorithm is presented hereinafter.

- The second one requires an advanced algorithm to optimize the trials for solving the high-dimension optimization problem defined by Eq. (44), the constraint $[z]^{T}[z]=\left[I_{m}\right]$ being automatically and exactly satisfied as described in [62].

- Efficient algorithm for generating realizations of the multivariate polynomial chaos in high dimension and for an arbitrary probability measure.

Let $\boldsymbol{\Psi}(\boldsymbol{\Xi})=\left(\Psi_{1}(\boldsymbol{\Xi}), \ldots, \Psi_{N}(\boldsymbol{\Xi})\right)$ be the $\mathbb{R}^{N}$-valued random vector in which $\left\{\Psi_{j}(\boldsymbol{\Xi})\right\}_{j=1}^{N}$ are the normalized multivariate Hermite polynomials. The objective is to compute the $\left(N \times \nu_{\text {chaos }}\right)$ real matrix $[\Psi]=\left[\Psi\left(\boldsymbol{\Xi}^{(1)}\right) \ldots \Psi\left(\boldsymbol{\Xi}^{\left(\nu_{\text {chaos }}\right)}\right)\right]$,

$$
[\Psi]=\left[\begin{array}{ccc}
\Psi_{1}\left(\boldsymbol{\Xi}^{(1)}\right) & \ldots & \Psi_{1}\left(\boldsymbol{\Xi}^{\left(\nu_{\text {chaos }}\right)}\right) \\
\cdot & \ldots & \cdot \\
\Psi_{N}\left(\boldsymbol{\Xi}^{(1)}\right) & \ldots & \Psi_{N}\left(\boldsymbol{\Xi}^{\left(\nu_{\text {chaos }}\right)}\right)
\end{array}\right],
$$

of the $\nu_{\text {chaos }}$ independent realizations $\Psi\left(\boldsymbol{\Xi}^{(1)}\right), \ldots, \boldsymbol{\Psi}\left(\boldsymbol{\Xi}^{\left(\nu_{\text {chaos }}\right)}\right)$, in preserving the orthogonality properties

$$
\lim _{\nu_{\text {chaos } \rightarrow+\infty}} \frac{1}{\nu_{\text {chaos }}}[\Psi][\Psi]^{T}=\left[I_{N}\right] .
$$

It should be noted that the algorithm, which is used for the Gaussian chaos $\Psi_{j}(\boldsymbol{\Xi})=$ $\Phi_{\alpha_{1}}\left(\Xi_{1}\right) \times \ldots \times \Phi_{\alpha_{N_{g}}}\left(\Xi_{N_{g}}\right)$ for $j=1, \ldots, N$, can also be used for an arbitrary non separable probability distribution $p_{\Xi}(\boldsymbol{\xi}) d \boldsymbol{\xi}$ on $\mathbb{R}^{N_{g}}$ without any modification, 
but in such a case, the multivariate polynomials $\left\{\Psi_{j}(\boldsymbol{\Xi})\right\}_{j=1}^{N}$, which verify the orthogonality property, $E\left\{\Psi_{j}(\boldsymbol{\Xi}) \Psi_{j^{\prime}}(\boldsymbol{\Xi})\right\}=\int_{\mathbb{R}^{N_{g}}} \Psi_{j}(\boldsymbol{\xi}) \Psi_{j^{\prime}}(\boldsymbol{\xi}) p_{\boldsymbol{\Xi}}(\boldsymbol{\xi}) d \boldsymbol{\xi}=\delta_{j j^{\prime}}$, are not written as a tensorial product of univariate polynomials (we have not $\Psi_{j}(\boldsymbol{\Xi})=\Phi_{\alpha_{1}}\left(\Xi_{1}\right) \times \ldots \times \Phi_{\alpha_{N_{g}}}\left(\Xi_{N_{g}}\right)$ ). It has been proved in [63] that, for the usual probability measure, the use of the explicit algebraic formula (constructed with a symbolic Toolbox) or the use of the computational recurrence relation with respect to the degree, induces important numerical noise and the orthogonality property is lost. In addition, if a global orthogonalization was done to correct this loss of orthogonality, then the independence of the realizations would be lost. A robust computational method has been proposed in $[63 ; 49]$ to preserve the orthogonality properties and the independence of the realizations. The two main steps are the following.

(i) Using a generator of independent realizations of $\boldsymbol{\Xi}$ whose probability distribution is $p_{\Xi}(\boldsymbol{\xi}) d \boldsymbol{\xi}$, the realizations $\mathcal{M}_{j}\left(\boldsymbol{\Xi}^{(1)}\right), \ldots, \mathcal{M}_{j}\left(\boldsymbol{\Xi}^{\left(\nu_{\text {chaos }}\right)}\right)$ of the multivariate monomials $\mathcal{M}_{j}(\boldsymbol{\Xi})=\Xi_{1}^{j_{1}} \times \ldots \times \Xi_{N_{g}}^{j_{N_{g}}}$ are computed, in which $j=$ $1, \ldots, N$ is the index associated with the multi-index $\left(j_{1}, \ldots, j_{N_{g}}\right)$. Let $\mathcal{M}(\boldsymbol{\Xi})=$ $\left(\mathcal{M}_{1}(\boldsymbol{\Xi}), \ldots, \mathcal{M}_{N}(\boldsymbol{\Xi})\right)$ be the $\mathbb{R}^{N}$-valued random variable and let $[M]$ be the $\left(N \times \nu_{\text {chaos }}\right)$ real matrix such that

$$
[M]=\left[\mathcal{M}\left(\boldsymbol{\Xi}^{(1)}\right) \ldots \mathcal{M}\left(\boldsymbol{\Xi}^{\left(\nu_{\text {chaos }}\right)}\right)\right]=\left[\begin{array}{ccc}
\mathcal{M}_{1}\left(\boldsymbol{\Xi}^{(1)}\right) & \ldots & \mathcal{M}_{1}\left(\boldsymbol{\Xi}^{\left(\nu_{\text {chaos }}\right)}\right) \\
\cdot & \ldots & \cdot \\
\mathcal{M}_{N}\left(\boldsymbol{\Xi}^{(1)}\right) & \ldots & \mathcal{M}_{N}\left(\boldsymbol{\Xi}^{\left(\nu_{\text {chaos }}\right)}\right)
\end{array}\right]
$$

(ii) An orthogonalization of the realizations of the multivariate monomials is carried out using an algorithm (that is different from the Gram-Schmidt orthogonalization algorithm, which is not stable in high dimension) based on the fact that: (a) the matrix $[\Psi]$, defined by Eq. (45), can be written as $[\Psi]=[A][M]$ in which $[A]$ is an invertible $(N \times N)$ real matrix and where $[M]$ is defined by Eq. (47), and (b) the matrix $[R]=E\left\{\mathcal{M}(\Xi) \mathcal{M}(\boldsymbol{\Xi})^{T}\right\}$ is written as $[R]=$ $\lim _{\nu_{\text {chaos }} \rightarrow+\infty} \frac{1}{\nu_{\text {chaos }}}[M][M]^{T}=[A]^{-1}[A]^{-T}$. The algorithm is summarized as follows:

- Computing matrix $[M]$ and then $[R] \simeq \frac{1}{\nu_{\text {chaos }}}[M][M]^{T}$ for $\nu_{\text {chaos }}$ sufficiently high.

- Computing $[A]^{-T}$ that corresponds to the Cholesky decomposition of $[R]$.

- Computing the lower triangular matrix $[A]$.

- Computing $[\Psi]=[A][M]$.

- Identification of truncation parameters $N_{d}$ and $N_{g}$. 
The quantification of the mean-square convergence of $\boldsymbol{\eta}^{\text {chaos }}\left(N_{d}, N_{g}\right)=\left[z_{0}\left(N_{d}\right.\right.$, $\left.\left.N_{g}\right)\right]^{T} \boldsymbol{\Psi}(\boldsymbol{\Xi})$ towards $\boldsymbol{\eta}^{\text {OAPSM }}$ with respect to $N_{d}$ and $N_{g}$, in which $\left[z_{0}\left(N_{d}, N_{g}\right)\right]$ is given by Eq. (44), is carried out using the $L^{1}-\log$ error function introduced in [62], which allows for measuring the errors of the small values of the probability density function (the tails of the pdf).

(i) For a fixed value of $N_{d} \leq m$ and $N_{g}$, and for $i=1, \ldots, m$ :

- Let $e \mapsto p_{\eta_{i}^{\text {OAPM }}}(e)$ be the pdf of random variable $\eta_{i}^{\text {OAPSM }}$, which is estimated with the one-dimensional kernel density estimation method using the independent realizations $\boldsymbol{\eta}^{(1)}, \ldots, \boldsymbol{\eta}^{\left(\nu_{\mathrm{KL}}\right)}$ of the random vector $\boldsymbol{\eta}^{\text {OAPSM }}$.

- Let $e \mapsto p_{\eta_{i}^{\text {chaos }}\left(N_{d}, N_{g}\right)}\left(e ;\left[z_{0}\left(N_{d}, N_{g}\right)\right]\right)$ be the pdf of random variable $\eta_{i}^{\text {chaos }}\left(N_{d}\right.$, $N_{g}$ ), which is estimated with the one-dimensional kernel density estimation method using $\nu_{\text {chaos }}$ independent realizations, $\boldsymbol{\eta}^{\text {chaos }(1)}\left(N_{d}, N_{g}\right), \ldots, \boldsymbol{\eta}^{\text {chaos }\left(\nu_{\text {chaos }}\right)}\left(N_{d}, N_{g}\right)$, of random vector $\boldsymbol{\eta}^{\text {chaos }}\left(N_{d}, N_{g}\right)$, which are such that $\boldsymbol{\eta}^{\text {chaos }(\ell)}\left(N_{d}, N_{g}\right)=\left[z_{0}\left(N_{d}\right.\right.$, $\left.\left.N_{g}\right)\right]^{T} \boldsymbol{\Psi}\left(\boldsymbol{\Xi}^{(\ell)}\right)$ in which $\boldsymbol{\Xi}^{(1)}, \ldots, \boldsymbol{\Xi}^{\left(\nu_{\text {chaos }}\right)}$ are $\nu_{\text {chaos }}$ independent realizations of $\boldsymbol{\Xi}$.

- The $L^{1}-\log$ error is introduced as described in [62]:

$$
\operatorname{err}_{i}\left(N_{d}, N_{g}\right)=\int_{\mathrm{BI}_{i}}\left|\log _{10} p_{\eta_{i}^{\text {OAPSM }}}(e)-\log _{10} p_{\eta_{i}^{\text {chaos }}\left(N_{d}, N_{g}\right)}\left(e ;\left[z_{0}\left(N_{d}, N_{g}\right)\right]\right)\right| d e,
$$

in which $\mathrm{BI}_{i}$ is a bounded interval of the real line, which is defined as the support of the one-dimensional kernel density estimator of random variable $\eta_{i}^{\text {OAPSM }}$, and which is then adapted to independent realizations $\boldsymbol{\eta}^{(1)}, \ldots, \boldsymbol{\eta}^{\left(\nu_{\mathrm{KL}}\right)}$ of $\boldsymbol{\eta}^{\text {OAPSM }}$.

(ii) For random vector $\boldsymbol{\eta}^{\text {chaos }}\left(N_{d}, N_{g}\right)$, the $L^{1}$-log error function is denoted by $\operatorname{err}\left(N_{d}, N_{g}\right)$ and is defined by

$$
\operatorname{err}\left(N_{g}, N_{d}\right)=\frac{1}{m} \sum_{i=1}^{m} \operatorname{err}_{i}\left(N_{d}, N_{g}\right)
$$

(iii) The optimal values $N_{d}^{\text {opt }}$ and $N_{g}^{\text {opt }}$ of the truncation parameters $N_{d}$ and $N_{g}$ are determined for minimizing the error function $\operatorname{err}\left(N_{d}, N_{g}\right)$ in taking into account the admissible set for the values of $N_{d}$ and $N_{g}$ as described in [49]. Let $\mathcal{C}_{N_{d}, N_{g}}$ be the admissible set for the values of $N_{d}$ and $N_{g}$, which is defined by

$$
\mathcal{C}_{N_{d}, N_{g}}=\left\{\left(N_{d}, N_{g}\right) \in \mathbb{N}^{2} \mid N_{g} \leq m,\left(N_{d}+N_{g}\right) ! /\left(N_{d} ! N_{g} !\right)-1 \geq m\right\} .
$$

It should be noted the more the values of $N_{d}$ and $N_{g}$ are high, the bigger is the matrix $\left[z_{0}\left(N_{d}, N_{g}\right)\right]$, and thus, the more difficult it is to perform the numerical identification. Rather than directly minimizing error function $\operatorname{err}\left(N_{d}, N_{g}\right)$, it is 
more accurate to search for the optimal values of $N_{d}$ and $N_{g}$ that minimize the dimension of the projection basis, $\left(N_{d}+N_{g}\right) ! /\left(N_{d} ! N_{g} !\right)$. For a given error threshold $\varepsilon$, we then introduce the admissible set $\mathcal{C}_{\varepsilon}$ such that

$$
\mathcal{C}_{\varepsilon}=\left\{\left(N_{d}, N_{g}\right) \in \mathcal{C}_{N_{d}, N_{g}} \mid \operatorname{err}\left(N_{g}, N_{d}\right) \leq \varepsilon\right\},
$$

and the optimal values $N_{d}^{\text {opt }}$ and $N_{g}^{\text {opt }}$ are given as the solution of the optimization problem,

$$
\left(N_{d}^{\mathrm{opt}}, N_{g}^{\mathrm{opt}}\right)=\arg \min _{\left(N_{d}, N_{g}\right) \in \mathcal{C}_{\varepsilon}}\left(N_{d}+N_{g}\right) ! /\left(N_{d} ! N_{g} !\right) \quad, \quad N^{\mathrm{opt}}=\llbracket h\left(N_{d}^{\mathrm{opt}}, N_{g}^{\mathrm{opt}}\right) .
$$

- Changing the notation.

Until the end of Step 5 and in Step 6 and Step 7, in order to simplify the notations, $N_{d}^{\text {opt }}, N_{g}^{\text {opt }}, N^{\text {opt }}$, and $\left[z_{0}\left(N_{d}^{\text {opt }}, N_{g}^{\text {opt }}\right)\right]$ are simply rewritten as $N_{d}, N_{g}, N$, and $\left[z_{0}\right]$.

\section{- Representation of random field $\left[\mathbf{K}^{\mathrm{OAPS}}\right]$.}

It can then be deduced that the optimal representation $\left\{\left[\mathbf{K}^{\operatorname{OAPSM}\left(m, N, N_{g}\right)}(\mathbf{x})\right], \mathbf{x} \in\right.$ $\Omega\}$ of random field $\left\{\left[\mathbf{K}^{\mathrm{OAPSM}}(\mathbf{x})\right], \mathbf{x} \in \Omega\right\}$ is written as

$$
\left[\mathbf{K}^{\operatorname{OAPSM}\left(m, N, N_{g}\right)}(\mathbf{x})\right]=\mathcal{K}^{\left(m, N, N_{g}\right)}\left(\mathbf{x}, \boldsymbol{\Xi},\left[z_{0}\right]\right) \quad, \quad \forall \mathbf{x} \in \Omega,
$$

in which $\mathcal{K}^{\left(m, N, N_{g}\right)}\left(\mathbf{x}, \boldsymbol{\Xi},\left[z_{0}\right]\right)$ is defined by Eq. (30) with $N_{d}=N_{d}^{\text {opt }}, N_{g}=N_{g}^{\text {opt }}$, and $[z]=\left[z_{0}\left(N_{d}^{\mathrm{opt}}, N_{g}^{\mathrm{opt}}\right]\right)$.

Step 6: Identification of the prior stochastic model $\left[K^{\text {prior }}\right]$ of $[K]$ in the general class of the non-Gaussian random fields.

This step consists in identifying the prior stochastic model $\left\{\left[\mathbf{K}^{\text {prior }}(\mathbf{x})\right], \mathbf{x} \in \Omega\right\}$ of $\{[\mathbf{K}(\mathbf{x})], \mathbf{x} \in \Omega\}$, using the maximum likelihood method and the experimental data sets $\mathbf{u}^{\exp , 1}, \ldots, \mathbf{u}^{\exp , \nu_{\exp }}$ relative to the random model observation $\mathbf{U}$ of the stochastic computational model (see Eq. (9)) and using the parametric modelbased representation of random observation model $\mathbf{U}$ (see Eq. (32)). We thus have to identify the value $\left[z^{\text {prior }}\right]$ in $\mathbb{V}_{m}\left(\mathbb{R}^{N^{\text {opt }}}\right)$ of $[z]$ such that

$$
\left[z^{\text {prior }}\right]=\arg \max _{[z] \in \mathbb{V}_{m}\left(\mathbb{R}^{N}\right)} \sum_{\ell=1}^{\nu_{\exp }} \log p_{\mathbf{U}^{\left(m, N, N_{g}\right)}}\left(\mathbf{u}^{\exp , \ell} ;[z]\right),
$$

in which $p_{\mathbf{U}^{(m, N, N g)}}\left(\mathbf{u}^{\exp , \ell} ;[z]\right)$ is the value, in $\mathbf{u}=\mathbf{u}^{\exp , \ell}$, of the pdf $p_{\mathbf{U}^{(m, N, N g)}}(\mathbf{u} ;[z])$ of the random vector $\mathbf{U}^{\left(m, N, N_{g}\right)}$ given (see Eq. (32)) by

$$
\mathbf{U}^{\left(m, N, N_{g}\right)}=\boldsymbol{B}^{\left(m, N, N_{g}\right)}(\boldsymbol{\Xi},[z])
$$


where $(\boldsymbol{\xi},[z]) \mapsto \mathcal{B}^{\left(m, N, N_{g}\right)}(\boldsymbol{\xi},[z])$ is the deterministic mapping from $\mathbb{R}^{N_{g}} \times$ $\mathbb{V}_{m}\left(\mathbb{R}^{N}\right)$ into $\mathbb{R}^{N_{U}}$ defined by Eq. (33) with Eq. (31) in which $\left[G_{0}(\mathbf{x})\right], \lambda_{i}$, and $\left[G_{i}(\mathbf{x})\right]$, for $i=1, \ldots, m$, are the quantities computed in Step 4 .

(i) For $[z]$ fixed in $\mathbb{V}_{m}\left(\mathbb{R}^{N}\right)$, pdf $\mathbf{u} \mapsto p_{\mathbf{U}^{\left(m, N, N_{g}\right)}}(\mathbf{u} ;[z])$ of random variable $\mathbf{U}^{\left(m, N, N_{g}\right)}$ is estimated by the multidimensional kernel density estimation method using $\nu_{\text {chaos }}$ independent realizations $\boldsymbol{\Xi}^{(1)}, \ldots, \boldsymbol{\Xi}^{\left(\nu_{\text {chaos }}\right)}$ of $\boldsymbol{\Xi}$.

(ii) Let us assume that $N>m$ is generally the case. The parameterization $[z]=\mathcal{R}_{\left[z_{0}\right]}([w])$ defined by Eq. (29) is used for exploring, with a random search algorithm, the subset of $\mathbb{V}_{m}\left(\mathbb{R}^{N}\right)$, centered in $\left[z_{0}\right]:=\left[z_{0}\left(N_{d}, N_{g}\right)\right] \in \mathbb{V}_{m}\left(\mathbb{R}^{N}\right)$ computed in Step 5. The optimization problem defined by Eq. (51) is replaced by $\left[z^{\text {prior }}\right]=\mathcal{R}_{\left[z_{0}\right]}\left(\left[w^{\text {prior }}\right]\right)$ with

$$
\left[w^{\text {prior }}\right]=\arg \max _{[w] \in T_{\left[z_{0}\right]}} \sum_{\ell=1}^{\nu_{\exp }} \log p_{\mathbf{U}^{\left(m, N, N_{g}\right)}}\left(\mathbf{u}^{\exp , \ell} ; \mathcal{R}_{\left[z_{0}\right]}([w])\right) .
$$

For solving the high-dimension optimization problem defined by Eq. (53), a random search algorithm is used for which $[w]$ is modeled by a random matrix $[\mathbf{W}]=\operatorname{Proj}_{T_{\left[z_{0}\right]}}([\boldsymbol{\Lambda}])$ with values in $T_{\left[z_{0}\right]}$, which is the projection on $T_{\left[z_{0}\right]}$ of a random matrix $[\boldsymbol{\Lambda}]$ with values in $\mathbb{M}_{N, m}(\mathbb{R})$ whose entries are independent normalized Gaussian real-valued random variables, i.e. $E\left\{[\boldsymbol{\Lambda}]_{j i}\right\}=0$ and $E\left\{[\boldsymbol{\Lambda}]_{j i}^{2}\right\}=1$. The positive parameter $\sigma$ introduced in Eq. (29) allows for controlling the "diameter" of the subset (centered in $\left[z_{0}\right]$ ) that is explored by the random search algorithm.

(iii) The representation of the prior stochastic model $\left\{\left[\mathbf{K}^{\text {prior }\left(m, N, N_{g}\right)}(\mathbf{x})\right], \mathbf{x} \in \Omega\right\}$ of random field $\{[\mathbf{K}(\mathbf{x})], \mathbf{x} \in \Omega\}$ is given by Eqs. (30) and (31) that are rewritten as

$$
\left[\mathbf{K}^{\text {prior }\left(m, N, N_{g}\right)}(\mathbf{x})\right]=\mathcal{K}^{\left(m, N, N_{g}\right)}\left(\mathbf{x}, \boldsymbol{\Xi},\left[z^{\text {prior }}\right]\right) \quad, \quad \forall \mathbf{x} \in \Omega,
$$

in which $\left[z^{\text {prior }}\right]$ is given by Eq. (51) and where $\mathcal{K}^{\left(m, N, N_{g}\right)}\left(\mathbf{x}, \boldsymbol{\xi},\left[z^{\text {prior }}\right]\right)$ is defined by Eq.(31) with $[z]=\left[z^{\text {prior }}\right]$.

Step 7: Identification of a posterior stochastic model $\left[K^{\text {post }}\right]$ of $[\mathbf{K}]$.

(i) A posterior stochastic model $\left\{\left[\mathbf{K}^{\text {post }}(\mathbf{x})\right], \mathbf{x} \in \Omega\right\}$ of random field $\{[\mathbf{K}(\mathbf{x})], \mathbf{x} \in$ $\Omega\}$ can be constructed using the Bayesian method. In such a framework, the coefficients $[z]$ of the polynomial chaos expansion $\boldsymbol{\eta}^{\text {chaos }}\left(N_{d}, N_{g}\right)=[z]^{T} \boldsymbol{\Psi}(\boldsymbol{\Xi})$ (see Eq. (41)) are modeled by a random matrix $[\mathbf{Z}]$ (see [61]) as proposed in [64] and consequently, $[z]$ is modeled by a $\mathbb{V}_{m}\left(\mathbb{R}^{N}\right)$-valued random variable $[\mathbf{Z}]$. The prior model $\left[\mathbf{Z}^{\text {prior }}\right]$ of $[\mathbf{Z}]$ is chosen as

$$
\left[\mathbf{Z}^{\text {prior }}\right]=\mathcal{R}_{\left[z^{\text {prior }}\right]}\left(\left[\mathbf{W}^{\text {prior }}\right]\right),
$$


in which $\mathcal{R}_{\left[z^{\text {pror }}\right]}$ is the mapping defined by Eq. (29), where $\left[z^{\text {prior }}\right]$ has been calculated in Step 6, and where $\left[\mathbf{W}^{\text {prior }}\right]=\operatorname{Proj}_{T_{[z \text { prior }}}\left(\left[\boldsymbol{\Lambda}^{\text {prior }}\right]\right)$ is a random matrix with values in $T_{\left[z^{\text {pror }}\right]}$, which is the projection on $T_{\left[z^{\text {pror }}\right]}$ of a random matrix $\left[\boldsymbol{\Lambda}^{\text {prior }}\right]$ with values in $\mathbb{M}_{N, m}(\mathbb{R})$ whose entries are independent normalized Gaussian realvalued random variables, i.e. $E\left\{[\boldsymbol{\Lambda}]_{j i}\right\}=0$ and $E\left\{[\boldsymbol{\Lambda}]_{j i}^{2}\right\}=1$. For a sufficiently small value of $\sigma$, the statistical fluctuations of the $\mathbb{V}_{m}\left(\mathbb{R}^{N^{\text {opt }}}\right)$-valued random matrix $\left[\mathbf{Z}^{\text {prior }}\right]$ are approximatively centered around $\left[z^{\text {prior }}\right]$. The Bayesian update allows the posterior distribution of the random matrix $\left[\mathbf{W}^{\text {post }}\right]$ with values in $T_{\left[z^{\text {prior }}\right]}$ to be estimated using the stochastic solution $\mathbf{U}^{\left(m, N, N_{g}\right)}=\mathcal{B}^{\left(m, N, N_{g}\right)}(\boldsymbol{\Xi}$,

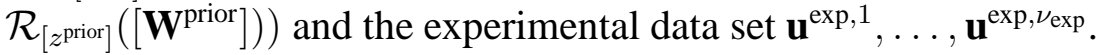

(ii) The representation of the posterior stochastic model $\left\{\left[\mathbf{K}^{\text {post }\left(m, N, N_{g}\right)}(\mathbf{x})\right], \mathbf{x} \in\right.$ $\Omega\}$ of random field $\{[\mathbf{K}(\mathbf{x})], \mathbf{x} \in \Omega\}$ is given by Eqs. (30) and (31) that are rewritten as

$$
\left[\mathbf{K}^{\text {post }\left(m, N, N_{g}\right)}(\mathbf{x})\right]=\mathcal{K}^{\left(m, N, N_{g}\right)}\left(\mathbf{x}, \boldsymbol{\Xi}, \mathcal{R}_{\left[z^{\text {prior }}\right]}\left(\left[\mathbf{W}^{\text {post }}\right]\right)\right) \quad, \quad \forall \mathbf{x} \in \Omega,
$$

in which $\mathcal{K}^{\left(m, N^{\text {opt }}, N_{g}^{\text {opt }}\right)}$ is defined by Eq. (31).

(iii) Once the probability distribution of $\left[\mathbf{W}^{\text {post }}\right]$ has been estimated by Step 7 , $\nu_{\mathrm{KL}}$ independent realizations can be calculated for the random field $\left[\mathbf{G}^{\text {post }}(\mathbf{x})\right]=$ $\left[G_{0}(\mathbf{x})\right]+\sum_{i=1}^{m} \sqrt{\sigma_{i}}\left[G_{i}(\mathbf{x})\right] \eta_{i}^{\text {post }}$ in which $\boldsymbol{\eta}^{\text {post }}=\left[\mathbf{Z}^{\text {post }}\right]^{T} \boldsymbol{\Psi}(\boldsymbol{\Xi})$ and where $\left[\mathbf{Z}^{\text {post }}\right]=$ $\mathcal{R}_{\left[z^{\text {prior }}\right]}\left(\left[\mathbf{W}^{\text {post }}\right]\right)$. The identification procedure can then be restarted from Step 4 replacing $\left[\mathbf{G}^{\mathrm{OAPSM}}\right]$ by $\left[\mathbf{G}^{\text {post }}\right]$.

\section{Construction of a Family of Algebraic Prior Stochastic Models}

We present an explicit construction of a family $\left\{\left[\mathbf{K}^{\mathrm{APSM}}(\mathbf{x} ; \mathbf{s})\right], \mathbf{x} \in \Omega\right\}$ of algebraic prior stochastic models for the non-Gaussian second-order random field $[\mathbf{K}]$ indexed by $\Omega$, with values in $\mathbb{M}_{n}^{+}(\mathbb{R})$, which has been introduced in Step 1 of Section "Methodology for Solving the Statistical Inverse Problem in High Stochastic Dimension". This family depends on a hyperparameter $\mathbf{s}$ belonging to the admissible set $\mathcal{C}_{s}$ that is a subset of $\mathbb{R}^{N_{s}}$, for which the dimension, $N_{s}$, is assumed to be relatively small, while the stochastic dimension of $\left[\mathbf{K}^{\mathrm{APSM}}\right]$ is high. For $\mathbf{s}$ fixed in $\mathcal{C}_{s}$, we give a construction of the random field $\left[\mathbf{K}^{\mathrm{APSM}}\right]$ and the corresponding generator of its realizations. In order to simplify the notations, $\mathbf{S}$ will be omitted as long as no confusion is possible. The formalism and the results, presented in Section "Nonparametric Stochastic Model for Constitutive Equation in Linear Elasticity" of "Random Matrix Models and Nonparametric Method for Uncertainty Quantification" in part II of the present Handbook on Uncertainty Quantification, are reused. Two prior algebraic stochastic models $\left[\mathbf{K}^{\mathrm{APSM}}\right]$ are presented hereinafter. 
- The first one is the algebraic prior stochastic model $\left[\mathbf{K}^{\mathrm{APSM}}\right]$ for the nonGaussian positive-definite matrix-valued random field $[\mathbf{K}]$ that exhibits anisotropic statistical fluctuations (initially introduced in $[56 ; 58]$ ), and for which there is a parameterization with a maximum of $d \times n(n+1) / 2$ spatial-correlation lengths and for which a positive-definite lower bound is given [60;65]. An extension of this model can be found in [28] for the case for which some positive-definite lower and upper bounds are introduced as constraints.

- The second one is the algebraic prior stochastic model $\left[\mathbf{K}^{\mathrm{APSM}}\right]$ described in [32; 34] for the non-Gaussian positive-definite matrix-valued random field $[\mathbf{K}]$ that exhibits (i) dominant statistical fluctuations in a symmetry class $\mathbb{M}_{n}^{\operatorname{sym}}(\mathbb{R}) \subset$ $\mathbb{M}_{n}^{+}(\mathbb{R})$ of dimension $N$ (isotropic, cubic, transversal isotropic, tetragonal, trigonal, orthotropic, monoclinic) for which there is a parameterization with $d N$ spatial-correlation lengths, (ii) anisotropic statistical fluctuations for which there is a parameterization with a maximum of $d \times n(n+1) / 2$ spatial-correlation lengths, and (iii) a positive-definite lower bound.

9.1. General Properties of the non-Gaussian Random Field $[\boldsymbol{K}]$ with a Lower Bound

Let $\{[\mathbf{K}(\mathbf{x})], \mathbf{x} \in \Omega\}$ be a non-Gaussian random defined on the probability space $(\Theta, \mathcal{T}, \mathcal{P})$, indexed by $\Omega \subset \mathbb{R}^{d}$ with $1 \leq d \leq 3$, with values in $\mathbb{M}_{n}^{+}(\mathbb{R})$ with $n=6$, homogeneous on $\mathbb{R}^{d}$, and of second-order, $E\left\{\|[\mathbf{K}(\mathbf{x})]\|_{F}^{2}\right\}<+\infty$ for all $\mathbf{x}$ in $\Omega$. Let $[\underline{K}] \in \mathbb{M}_{n}^{+}(\mathbb{R})$ be its mean value that is independent of $\mathbf{x}$ (homogeneous random field) and let $\left[C_{\ell}\right] \in \mathbb{M}_{n}^{+}(\mathbb{R})$ be its positive-definite lower bound that is also assumed to be independent of $\mathbf{x}$. For all $\mathbf{x}$ in $\Omega$,

$$
[\underline{K}]=E\{[\mathbf{K}(\mathbf{x})]\} \quad, \quad[\mathbf{K}(\mathbf{x})]-\left[C_{\ell}\right]>0 \quad \text { a.s. }
$$

9.2. Algebraic prior stochastic model for the case of anisotropic statistical fluctuations

We consider the case for which the random field exhibits anisotropic statistical fluctuations.

\section{Introduction of an adapted representation.}

The prior stochastic model $\left\{\left[\mathbf{K}^{\mathrm{APSM}}(\mathbf{x})\right], \mathbf{x} \in \Omega\right\}$ of the random field $\{[\mathbf{K}(\mathbf{x})], \mathbf{x} \in$ $\Omega\}$, is defined on $(\Theta, \mathcal{T}, \mathcal{P})$, is indexed by $\Omega \subset \mathbb{R}^{d}$, is with values in $\mathbb{M}_{n}^{+}(\mathbb{R})$, is homogeneous on $\mathbb{R}^{d}$, and is a second-order random field that is written as

$$
\left[\mathbf{K}^{\mathrm{APSM}}(\mathbf{x})\right]=\left[C_{\ell}\right]+[\underline{C}]^{1 / 2}\left[\mathbf{G}_{0}(\mathbf{x})\right][\underline{C}]^{1 / 2} \quad, \quad \forall \mathbf{x} \in \Omega,
$$


where $[\underline{C}]^{1 / 2}$ is the square root of the matrix $[\underline{C}]$ in $\mathbb{M}_{n}^{+}(\mathbb{R})$, independent of $\mathbf{x}$, defined by

$$
[\underline{C}]=[\underline{K}]-\left[C_{\ell}\right] \in \mathbb{M}_{n}^{+}(\mathbb{R}) .
$$

In Eq. (58), $\left\{\left[\mathbf{G}_{0}(\mathbf{x})\right], \mathbf{x} \in \mathbb{R}^{d}\right\}$ is a random field defined on $(\Theta, \mathcal{T}, \mathcal{P})$, indexed by $\mathbb{R}^{d}$, with values in $\mathbb{M}_{n}^{+}(\mathbb{R})$, homogeneous on $\mathbb{R}^{d}$, second-order such that, for all $\mathbf{x}$ in $\mathbb{R}^{d}$,

$$
E\left\{\left[\mathbf{G}_{0}(\mathbf{x})\right]\right\}=\left[I_{n}\right] \quad, \quad\left[\mathbf{G}_{0}(\mathbf{x})\right]>0 \quad \text { a.s. }
$$

It can then be deduced that, for all $\mathbf{x}$ in $\Omega$,

$$
E\left\{\left[\mathbf{K}^{\mathrm{APSM}}(\mathbf{x})\right]\right\}=[\underline{K}] \quad, \quad\left[\mathbf{K}^{\mathrm{APSM}}(\mathbf{x})\right]-\left[C_{\ell}\right]>0 \quad \text { a.s. }
$$

\section{Construction of random field $\left[G_{0}\right]$ and its generator of realizations.}

- Random fields $\mathcal{U}_{j k}$ as the stochastic germs of the random field $\left[\mathbf{G}_{0}\right]$.

Random field $\left\{\left[\mathbf{G}_{0}(\mathbf{x})\right], \mathbf{x} \in \mathbb{R}^{d}\right\}$ is constructed as a nonlinear transformation of $n(n+1) / 2$ independent second-order, centered, homogeneous, Gaussian, and normalized random fields $\left\{\mathcal{U}_{j k}(\mathbf{x}), \mathbf{x} \in \mathbb{R}^{d}\right\}_{1 \leq j \leq k \leq n}$, defined on probability space $(\Theta, \mathcal{T}, \mathcal{P})$, indexed by $\mathbb{R}^{d}$, with values in $\mathbb{R}$, and named the stochastic germs of the non-Gaussian random field $\left[\mathbf{G}_{0}\right]$. We then have

$$
E\left\{\mathcal{U}_{j k}(\mathbf{x})\right\}=0 \quad, \quad E\left\{\mathcal{U}_{j k}(\mathbf{x})^{2}\right\}=1
$$

Consequently, the random fields $\left\{\mathcal{U}_{j k}(\mathbf{x}), \mathbf{x} \in \mathbb{R}^{d}\right\}_{1 \leq j \leq k \leq n}$ are completely and uniquely defined by the $n(n+1) / 2$ autocorrelation functions $\zeta=\left(\zeta_{1}, \ldots, \zeta_{d}\right) \mapsto$ $R_{\mathcal{U}_{j k}}(\boldsymbol{\zeta})=E\left\{\mathcal{U}_{j k}(\mathbf{x}+\boldsymbol{\zeta}) \mathcal{U}_{j k}(\mathbf{x})\right\}$ from $\mathbb{R}^{d}$ into $\mathbb{R}$, such that $R_{\mathcal{U}_{j k}}(0)=1$. The spatial-correlation lengths $L_{1}^{j k}, \ldots, L_{d}^{j k}$ of random field $\left\{\mathcal{U}_{j k}(\mathbf{x}), \mathbf{x} \in \mathbb{R}^{d}\right\}$ are defined by

$$
L_{\alpha}^{j k}=\int_{0}^{+\infty}\left|R_{\mathcal{U}_{j k}}\left(0, \ldots, \zeta_{\alpha}, \ldots, 0\right)\right| d \zeta_{\alpha} \quad, \quad \alpha=1, \ldots d,
$$

and are generally chosen as parameters for the parameterization.

Example of parameterization for autocorrelation function $R_{\mathcal{U}_{j k}}$. The autocorrelation function (corresponding to a minimal parameterization) is written as

$$
R_{\mathcal{U}_{j k}}(\boldsymbol{\zeta})=\rho_{1}^{j k}\left(\zeta_{1}\right) \times \ldots \times \rho_{d}^{j k}\left(\zeta_{d}\right),
$$

in which, for all $\alpha=1, \ldots, d, \rho_{\alpha}^{j k}(0)=1$, and for all $\zeta_{\alpha} \neq 0$,

$$
\rho_{\alpha}^{j k}\left(\zeta_{\alpha}\right)=4\left(L_{\alpha}^{j k}\right)^{2} /\left(\pi^{2} \zeta_{\alpha}^{2}\right) \sin ^{2}\left(\pi \zeta_{\alpha} /\left(2 L_{\alpha}^{j k}\right)\right),
$$


where $L_{1}^{j k}, \ldots, L_{d}^{j k}$ are positive real numbers. Each random field $\mathcal{U}_{j k}$ is meansquare continuous on $\mathbb{R}^{d}$ and its power spectral density function defined on $\mathbb{R}^{d}$ has a compact support, $\left[-\pi / L_{1}^{j k}, \pi / L_{1}^{j k}\right] \times \ldots \times\left[-\pi / L_{1}^{j k}, \pi / L_{d}^{j k}\right]$. Such a model has $d n(n+1) / 2$ real parameters $\left\{L_{1}^{j k}, \ldots, L_{d}^{j k}\right\}_{1 \leq j \leq k \leq n}$ that represent the spatialcorrelation lengths of the stochastic germs $\left\{\mathcal{U}_{j k}(\mathbf{x}), \mathbf{x} \in \mathbb{R}^{d}\right\}_{1 \leq j \leq k \leq n}$, because

$$
\left.\int_{0}^{+\infty}\left|R_{\mathcal{U}_{j k}}\left(0, \ldots, \zeta_{\alpha}, \ldots, 0\right)\right| d \zeta_{\alpha}\right)=L_{\alpha}^{j k}
$$

- Defining an adapted family of functions for the nonlinear transformation.

Let $\{u \mapsto h(u ; a)\}_{a>0}$ be the adapted family of functions from $\mathbb{R}$ into $] 0,+\infty[$, in which $a$ is a positive real number, such that $\Gamma_{a}=h(\mathcal{U} ; a)$ is a gamma random variable with parameter $a$ while $\mathcal{U}$ is a normalized Gaussian random variable $\left(E\{\mathcal{U}\}=0\right.$ and $\left.E\left\{\mathcal{U}^{2}\right\}=1\right)$. Consequently, for all $u$ in $\mathbb{R}$, we have

$$
h(u ; a)=F_{\Gamma_{a}}^{-1}\left(F_{\mathcal{U}}(u)\right),
$$

in which $u \mapsto F_{\mathcal{U}}(u)=\int_{-\infty}^{u} \frac{1}{\sqrt{2 \pi}} e^{-v^{2} / 2} d v$ is the cumulative distribution function of the normalized Gaussian random variable $\mathcal{U}$. The function $p \mapsto F_{\Gamma_{a}}^{-1}(p)$, from $] 0,1[$ into $] 0,+\infty$ [, is the reciprocal function of the cumulative distribution function $\gamma \mapsto F_{\Gamma_{a}}(\gamma)=\int_{0}^{\gamma} \frac{1}{\Gamma(a)} t^{a-1} e^{-t} d t$ of the gamma random variable $\Gamma_{a}$ with parameter $a$, in which $\Gamma(a)$ is the gamma function defined by $\Gamma(a)=$ $\int_{0}^{+\infty} t^{a-1} e^{-t} d t$.

- Defining the random field $\left\{\left[\mathbf{G}_{0}(\mathbf{x})\right], \mathbf{x} \in \mathbb{R}^{d}\right\}$ and its generator of realizations.

For all $\mathbf{x}$ fixed in $\mathbb{R}^{d}$, the available information is defined by Eq. (60)) and by the constraint $\left|E\left\{\log \left(\operatorname{det}\left[\mathbf{G}_{0}(\mathbf{x})\right]\right)\right\}\right|<+\infty$, which is introduced in order that the zero matrix be a repulsive value for the random matrix $\left[\mathbf{G}_{0}(\mathbf{x})\right]$. The use of the maximum entropy principle under the constraints defined by this available information leads to taking the random matrix $\left[\mathbf{G}_{0}(\mathbf{x})\right]$ in ensemble $\mathrm{SG}_{0}^{+}$defined in Section

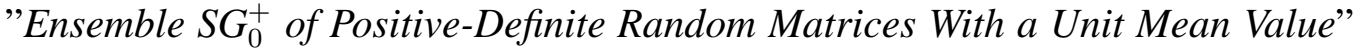
of "Random Matrix Models and Nonparametric Method for Uncertainty Quantification" in part II of the present Handbook on Uncertainty Quantification. Taking into account the algebraic representation of any random matrix belonging to ensemble $\mathrm{SG}_{0}^{+}$, the spatial-correlation structure of random field $\left[\mathbf{G}_{0}\right]$ is then introduced in replacing the Gaussian random variables $U_{j k}$ by the Gaussian real-valued random fields $\left\{\mathcal{U}_{j k}(\mathbf{x}), \mathbf{x} \in \mathbb{R}^{d}\right\}$ defined above, for which the spatial-correlation 
structure is defined by the spatial-correlation lengths $\left\{L_{\alpha}^{j k}\right\}_{\alpha=1, \ldots, d}$. Consequently, the random field $\left\{\left[\mathbf{G}_{0}(\mathbf{x})\right], \mathbf{x} \in \mathbb{R}^{d}\right\}$, defined on probability space $(\Theta, \mathcal{T}, \mathcal{P})$, indexed by $\mathbb{R}^{d}$, with values in $\mathbb{M}_{n}^{+}(\mathbb{R})$ is constructed as follows:

(i) Let $\left\{\mathcal{U}_{j k}(\mathbf{x}), \mathbf{x} \in \mathbb{R}^{d}\right\}_{1 \leq j \leq k \leq n}$ be the $n(n+1) / 2$ independent random fields introduced above. Consequently, for all $\mathbf{x}$ in $\mathbb{R}^{d}$,

$$
E\left\{\mathcal{U}_{j k}(\mathbf{x})\right\}=0 \quad, \quad E\left\{\mathcal{U}_{j k}(\mathbf{x})^{2}\right\}=1 \quad, \quad 1 \leq j \leq k \leq n
$$

(ii) Let $\delta$ be the real number, independent of $\mathbf{x}$, such that

$$
0<\delta<\sqrt{(n+1) /(n+5)}<1 .
$$

The parameter $\delta$ allows for controlling the statistical fluctuations (dispersion) of the random field $\left[\mathbf{G}_{0}\right]$.

(iii) For all $\mathbf{x}$ fixed in $\mathbb{R}^{d}$, the random matrix $\left[\mathbf{G}_{0}(\mathbf{x})\right]$ is written as

$$
\left[\mathbf{G}_{0}(\mathbf{x})\right]=[\mathbf{L}(\mathbf{x})]^{T}[\mathbf{L}(\mathbf{x})]
$$

in which $[\mathbf{L}(\mathbf{x})]$ is the upper $(n \times n)$ real triangular random matrix defined as follows:

- For $1 \leq j \leq k \leq n$, the $n(n+1) / 2$ random fields $\left\{[\mathbf{L}(\mathbf{x})]_{j k}, \mathbf{x} \in \Omega\right\}$ are independent.

- For $j<k$, the real-valued random field $\left\{[\mathbf{L}(\mathbf{x})]_{j k}, \mathbf{x} \in \Omega\right\}$ is defined by $[\mathbf{L}(\mathbf{x})]_{j k}=\sigma_{n} \mathcal{U}_{j k}(\mathbf{x})$ in which $\sigma_{n}$ is such that $\sigma_{n}=\delta / \sqrt{n+1}$.

- For $j=k$, the positive-valued random field $\left\{[\mathbf{L}(\mathbf{x})]_{j j}, \mathbf{x} \in \Omega\right\}$ is defined by $[\mathbf{L}(\mathbf{x})]_{j j}=\sigma_{n} \sqrt{2 h\left(\mathcal{U}_{j j}(\mathbf{x}), a_{j}\right)}$ in which $a_{j}=(n+1) /\left(2 \delta^{2}\right)+(1-j) / 2$.

(iv) The representation of random field $\left[\mathbf{G}_{0}\right]$ defined by Eq. (70) allows for computing realizations of the family of dependent random matrices $\left\{\left[\mathbf{G}_{0}\left(\mathbf{x}^{1}\right)\right], \ldots\right.$, $\left.\left[\mathbf{G}_{0}\left(\mathbf{x}^{N_{p}}\right)\right]\right\}$ in which $\mathbf{x}^{1}, \ldots, \mathbf{x}^{N_{p}}$ are $N_{p}$ given points in $\Omega$, which are expressed using the realizations of $\left\{\mathcal{U}_{j k}\left(\mathbf{x}^{1}\right), \ldots, \mathcal{U}_{j k}\left(\mathbf{x}^{N_{p}}\right)\right\}_{1 \leq j \leq k \leq n}$ that are simulated using either the representation adapted to a large value of $N_{p}$, or another one adapted to a small or moderate value of $N_{p}$ (see [58]).

- A few basic properties of random field $\left[\mathbf{G}_{0}\right]$.

The random field $\left\{\left[\mathbf{G}_{0}(\mathbf{x})\right], \mathbf{x} \in \Omega\right\}$, defined on $(\Theta, \mathcal{T}, \mathcal{P})$, indexed by $\mathbb{R}^{d}$, with values in $\mathbb{M}_{n}^{+}(\mathbb{R})$, is a homogeneous, second-order, and mean-square continuous random field. For all $\mathbf{x}$ in $\mathbb{R}^{d}$,

$$
E\left\{\left\|\mathbf{G}_{0}(\mathbf{x})\right\|_{F}^{2}\right\}<+\infty \quad, \quad E\left\{\left[\mathbf{G}_{0}(\mathbf{x})\right]\right\}=\left[I_{n}\right]
$$


It can be proved that the introduced dispersion parameter corresponds to the following definition

$$
\delta=\left\{\frac{1}{n} E\left\{\left\|\left[\mathbf{G}_{0}(\mathbf{x})\right]-\left[I_{n}\right]\right\|_{F}^{2}\right\}\right\}^{1 / 2}
$$

which shows that

$$
E\left\{\left\|\mathbf{G}_{0}(\mathbf{x})\right\|_{F}^{2}\right\}=n\left(\delta^{2}+1\right),
$$

in which $\delta$ is independent of $\mathbf{x}$. For all $\mathbf{x}$ fixed in $\mathbb{R}^{d}$, the probability density function with respect to the measure $d^{S} G=2^{n(n-1) / 4} \prod_{1 \leq j \leq k \leq n} d G_{j k}$ of random matrix $\left[\mathbf{G}_{0}(\mathbf{x})\right]$ is independent of $\mathbf{x}$ and is written as

$$
p_{\left[\mathbf{G}_{0}(\mathbf{x})\right]}([G])=\mathbb{1}_{\mathbb{M}_{n}^{+}(\mathbb{R})}([G]) \times C_{\mathbf{G}_{0}} \times(\operatorname{det}[G])^{(n+1) \frac{\left(1-\delta^{2}\right)}{2 \delta^{2}}} \times \exp \left\{-\frac{(n+1)}{2 \delta^{2}} \operatorname{tr}[G]\right\},
$$

where $C_{\mathbf{G}_{0}}$ is the positive constant of normalization. For all $\mathbf{x}$ fixed in $\mathbb{R}^{d}$, the random variables $\left\{\left[\mathbf{G}_{0}(\mathbf{x})\right]_{j k}, 1 \leq j \leq k \leq 6\right\}$ are mutually dependent. In addition, the system of the marginal probability distributions of random field $\left\{\left[\mathbf{G}_{0}(\mathbf{x})\right], \mathbf{x} \in \Omega\right\}$ is completely defined and is not Gaussian. There exists a positive constant $b_{G}$ independent of $\mathbf{x}$, but depending on $\delta$, such that for all $\mathbf{x}$ in $\mathbb{R}^{d}$,

$$
E\left\{\left\|\left[\mathbf{G}_{0}(\mathbf{x})\right]^{-1}\right\|^{2}\right\} \leq b_{G}<+\infty .
$$

Since $\left[\mathbf{G}_{0}(\mathbf{x})\right]$ is a random matrix with values in $\mathbb{M}_{n}^{+}(\mathbb{R})$, then $\left[\mathbf{G}_{0}(\mathbf{x})\right]^{-1}$ exists (almost surely). However, since almost sure convergence does not imply meansquare convergence, Eq. (75) cannot simply be deduced. Let $\bar{\Omega}=\Omega \cup \partial \Omega$ be the closure of the bounded set $\Omega$. We then have

$$
E\left\{\left(\sup _{\mathbf{x} \in \bar{\Omega}}\left\|\left[\mathbf{G}_{0}(\mathbf{x})\right]^{-1}\right\|\right)^{2}\right\}=c_{G}^{2}<+\infty,
$$

in which sup is the supremum and where $0<c_{G}<+\infty$ is a finite positive constant.

\section{Definition of the hyperparameter $s$.}

The hyperparameter $\mathbf{s} \in \mathcal{C}_{s} \subset \mathbb{R}^{N_{s}}$ of the $\operatorname{APSM}\left\{\left[\mathbf{K}^{\mathrm{APSM}}(\mathbf{x} ; \mathbf{s})\right], \mathbf{x} \in \Omega\right\}$ that has been constructed for the anisotropic statistical fluctuations, is constituted of:

- the reshaping of $\left[C_{\ell}\right] \in \mathbb{M}_{n}^{+}(\mathbb{R})$ (the lower bound) and $[\underline{K}] \in \mathbb{M}_{n}^{+}(\mathbb{R})$ (the mean value),

- the $d n(n+1) / 2$ positive real numbers, $\left\{L_{1}^{j k}, \ldots, L_{d}^{j k}\right\}_{1 \leq j \leq k \leq n}$ (the spatialcorrelation lengths, for the parameterization given in the example) and $\delta$ (the dispersion) such that $0<\delta<\sqrt{(n+1) /(n+5)}$. 
9.3. Algebraic prior stochastic model for the case of dominant statistical fluctuations in a symmetry class with some anisotropic statistical fluctuations

We now consider the case for which the random field exhibits dominant statistical fluctuations in a symmetry class and some anisotropic statistical fluctuations.

\section{Positive-definite matrices belonging to a symmetry class.}

A given symmetry class is defined by a subset $\mathbb{M}_{n}^{\text {sym }}(\mathbb{R})$ of $\mathbb{M}_{n}^{+}(\mathbb{R})$ such that any matrix $[M]$ belonging to $\mathbb{M}_{n}^{\text {sym }}(\mathbb{R})$ is written as

$$
[M]=\sum_{j=1}^{N} m_{j}\left[E_{j}^{\mathrm{sym}}\right] \quad, \quad \mathbf{m}=\left(m_{1}, \ldots, m_{N}\right) \in \mathcal{C}_{\mathbf{m}} \subset \mathbb{R}^{N} \quad, \quad\left[E_{j}^{\mathrm{sym}}\right] \in \mathbb{M}_{n}^{S}(\mathbb{R}),
$$

in which $\left\{\left[E_{j}^{\text {sym }}\right], j=1, \ldots, N\right\}$ is the matrix basis of $\mathbb{M}_{n}^{\text {sym }}(\mathbb{R})$ (Walpole's tensor basis [75] in the framework of the elasticity theory), and where the admissible subset $\mathcal{C}_{\mathbf{m}}$ of $\mathbb{R}^{N}$ is defined by

$$
\mathcal{C}_{\mathbf{m}}=\left\{\mathbf{m} \in \mathbb{R}^{N} \mid \sum_{j=1}^{N} m_{j}\left[E_{j}^{\mathrm{sym}}\right] \in \mathbb{M}_{n}^{+}(\mathbb{R})\right\}
$$

It should be noted that matrices $\left[E_{1}^{\mathrm{sym}}\right], \ldots,\left[E_{N}^{\mathrm{sym}}\right]$ are symmetric but are not positive definite. For the usual material symmetry classes, the possible values of $N$ are the following: 2 for isotropic, 3 for cubic, 5 for transversely isotropic, 6 or 7 for tetragonal, 6 or 7 for trigonal, 9 for orthotropic, 13 for monoclinic, and 21 for anisotropic. The following properties are proved (see $[75 ; 33])$ :

(i) If $[M]$ and $\left[M^{\prime}\right]$ belong to $\mathbb{M}_{n}^{\text {sym }}(\mathbb{R})$, then for all $a$ and $b$ in $\mathbb{R}, a[M]+b\left[M^{\prime}\right] \in$ $\mathbb{M}_{n}^{\text {sym }}(\mathbb{R})$, and

$$
[M]\left[M^{\prime}\right] \in \mathbb{M}_{n}^{\text {sym }}(\mathbb{R}) \quad, \quad[M]^{-1} \in \mathbb{M}_{n}^{\text {sym }}(\mathbb{R}) \quad, \quad[M]^{1 / 2} \in \mathbb{M}_{n}^{\text {sym }}(\mathbb{R}) .
$$

(ii) Any matrix $[N]$ belonging to $\mathbb{M}_{n}^{\text {sym }}(\mathbb{R})$ can be written as

$$
[N]=\exp _{\mathbb{M}}([\mathcal{N}]) \quad, \quad[\mathcal{N}]=\sum_{j=1}^{N} y_{j}\left[E_{j}^{\text {sym }}\right] \quad, \quad \mathbf{y}=\left(y_{1}, \ldots, y_{N}\right) \in \mathbb{R}^{N}
$$

in which $\exp _{\mathbb{M}}$ is the exponential of symmetric real matrices. It should be noted that matrix $[\mathcal{N}]$ is a symmetric real matrix but does not belong to $\mathbb{M}_{n}^{\text {sym }}(\mathbb{R}$ ) (because $\mathbf{y}$ is in $\mathbb{R}^{N}$ and not in $\mathcal{C}_{\mathbf{m}}$ and therefore, $[\mathcal{N}]$ is not positive definite). 
(iii) From Eqs. (77) and (80), it can be deduced that

$$
\exp _{\mathbb{M}}\left(\sum_{j=1}^{N} y_{j}\left[E_{j}^{\mathrm{sym}}\right]\right)=\sum_{j=1}^{N} m_{j}(\mathbf{y})\left[E_{j}^{\mathrm{sym}}\right] \quad, \quad \forall \mathbf{y} \in \mathbb{R}^{N},
$$

in which $\mathbf{m}(\mathbf{y})=\left(m_{1}(\mathbf{y}), \ldots, m_{N}(\mathbf{y})\right)$ belongs to $\mathcal{C}_{\mathbf{m}(\mathbf{y})}$ that is defined by Eq. (78). Let $[\mathcal{E}]$ be the matrix in $\mathbb{M}_{N}^{S}(\mathbb{R})$ such that $\left.[\mathcal{E}]_{k j}=\ll\left[E_{j}^{\text {sym }}\right],\left[E_{k}^{\text {sym }}\right]\right) \gg$ and let $\mathcal{F}(\mathbf{y})=\left(\mathcal{F}_{1}(\mathbf{y}), \ldots, \mathcal{F}_{N}(\mathbf{y})\right)$ be the vector in $\mathbb{R}^{N}$ such that

$$
\left.\mathcal{F}_{k}(\mathbf{y})=\ll \exp _{\mathbb{M}}\left(\sum_{j=1}^{N} y_{j}\left[E_{j}^{\mathrm{sym}}\right]\right),\left[E_{k}^{\mathrm{sym}}\right]\right) \gg .
$$

For all $\mathbf{y}$ fixed in $\mathbb{R}^{N}, \mathbf{m}(\mathbf{y})$ is the unique solution in $\mathcal{C}_{\mathbf{m}(\mathbf{y})}$ of the linear system,

$$
[\mathcal{E}] \mathbf{m}(\mathbf{y})=\mathcal{F}(\mathbf{y}) .
$$

It should be noted that, in the purely computational framework that is proposed in the previous section "Methodology for Solving the Statistical Inverse Problem in High Stochastic Dimension", an explicit calculation of $\mathcal{F}(\mathbf{y})$ is not required. For each numerical value of vector $\mathbf{y}$, vector $\mathbf{m}(\mathbf{y})$ is computed by solving the linear equation defined by Eq. (82) in which $\mathcal{F}(\mathbf{y})$ is numerically calculated.

Introduction of the matrices $[\underline{C}],[\underline{S}]$, and $[\underline{A}]$ related to the mean value of the matrix-valued random field.

Let $[\underline{C}]$ be the matrix in $\mathbb{M}_{n}^{+}(\mathbb{R})$, independent of $\mathbf{x}$, representing the mean value of the random matrix $[\mathbf{C}(\mathbf{x})]=[\mathbf{K}(\mathbf{x})]-\left[C_{\ell}\right]$. From Eq. (57), it can then be deduced that

$$
[\underline{C}]=[\underline{K}]-\left[C_{\ell}\right] \in \mathbb{M}_{n}^{+}(\mathbb{R}) .
$$

Let $[\underline{A}]$ be the deterministic matrix in $\mathbb{M}_{n}^{\text {sym }}(\mathbb{R})$, independent of $\mathbf{x}$, representing the projection of the mean matrix $[\underline{C}]$ on the symmetry class $\mathbb{M}_{n}^{\text {sym }}(\mathbb{R})$,

$$
[\underline{A}]=P^{\mathrm{sym}}([\underline{C}]) \in \mathbb{M}_{n}^{\mathrm{sym}}(\mathbb{R}),
$$

in which $[\underline{C}]$ is defined by Eq. (83) and where $P^{\text {sym }}$ is the projection operator from $\mathbb{M}_{n}^{+}(\mathbb{R})$ onto $\mathbb{M}_{n}^{\text {sym }}(\mathbb{R})$.

(i) For a random field with values in a given symmetry class with $N<21$ (there are no anisotropic statistical fluctuations), the matrices $[\underline{K}]$ and $\left[C_{\ell}\right]$ belong to the symmetry class and consequently, $[\underline{C}]$ must belong to $\mathbb{M}_{n}^{\text {sym }}(\mathbb{R})$, and thus, $[\underline{A}]$ is equal to $[\underline{C}]$. 
(ii) If the class of symmetry is anisotropic (thus $N=21$ ), then $\mathbb{M}_{n}^{\text {sym }}(\mathbb{R})$ coincides with $\mathbb{M}_{n}^{+}(\mathbb{R})$ and again, $[\underline{A}]$ is equal to the mean matrix $[\underline{C}]$ that belongs to $\mathbb{M}_{n}^{+}(\mathbb{R})$. (iii) In general, for a given symmetry class with $N<21$, and due to the presence of anisotropic statistical fluctuations, the mean value $[\underline{C}]$ of the random matrix $[\mathbf{C}(\mathbf{x})]=[\mathbf{K}(\mathbf{x})]-\left[C_{\ell}\right]$ belongs to $\mathbb{M}_{n}^{+}(\mathbb{R})$ but does not belong to $\mathbb{M}_{n}^{\text {sym }}(\mathbb{R})$. For this case, an invertible deterministic $(n \times n)$ real matrix $[\underline{S}]$ is introduced such that

$$
[\underline{C}]=[\underline{S}]^{T}[\underline{A}][\underline{S}] .
$$

The construction of $[\underline{S}]$ is performed as follows. Let $\left[L_{C}\right]$ and $\left[L_{A}\right]$ be the upper triangular real matrices with positive diagonal entries resulting from the Cholesky factorization of matrices $[\underline{C}]$ and $[\underline{A}]$,

$$
[\underline{C}]=\left[L_{\underline{C}}\right]^{T}\left[L_{\underline{C}}\right] \quad, \quad[\underline{A}]=\left[L_{\underline{A}}\right]^{T}\left[L_{\underline{A}}\right] .
$$

Therefore, the matrix $[\underline{S}]$ is defined by

$$
[\underline{S}]=\left[L_{\underline{A}}\right]^{-1}\left[L_{C}\right] \text {. }
$$

It should be noted that for cases (i) and (ii) above, Eq. (85) shows that $[\underline{S}]=\left[I_{n}\right]$.

Introduction of an adapted representation for the random field.

The prior stochastic model $\left\{\left[\mathbf{K}^{\mathrm{APSM}}(\mathbf{x})\right], \mathbf{x} \in \Omega\right\}$ of the second-order random field $\{[\mathbf{K}(\mathbf{x})], \mathbf{x} \in \Omega\}$, indexed by $\Omega \subset \mathbb{R}^{d}$, with values in $\mathbb{M}_{n}^{+}(\mathbb{R})$, is written as

$$
\left[\mathbf{K}^{\mathrm{APSM}}(\mathbf{x})\right]=\left[C_{\ell}\right]+[\underline{S}]^{T}[\mathbf{A}(\mathbf{x})]^{1 / 2}\left[\mathbf{G}_{0}(\mathbf{x})\right][\mathbf{A}(\mathbf{x})]^{1 / 2}[\underline{S}] \quad, \quad \forall \mathbf{x} \in \Omega .
$$

in which the deterministic $(n \times n)$ real matrix $[\underline{S}]$ is defined by Eq. (85), and where $\left\{\left[\mathbf{G}_{0}(\mathbf{x})\right], \mathbf{x} \in \Omega\right\}$ and $\{[\mathbf{A}(\mathbf{x})], \mathbf{x} \in \Omega\}$ are random fields indexed by $\mathbb{R}^{d}$ and homogeneous on $\mathbb{R}^{d}$. Consequently, the random field $\left\{\left[\mathbf{K}^{\mathrm{APSM}}(\mathbf{x})\right], \mathbf{x} \in \Omega\right\}$ that is indexed by $\Omega$, is the restriction to $\Omega \subset \mathbb{R}^{d}$ of a homogeneous random field.

- Anisotropic statistical fluctuations described by $\left\{\left[\boldsymbol{G}_{0}(\boldsymbol{x})\right], \boldsymbol{x} \in \mathbb{R}^{d}\right\}$.

The random field $\left\{\left[\mathbf{G}_{0}(\mathbf{x})\right], \mathbf{x} \in \mathbb{R}^{d}\right\}$ models the anisotropic statistical fluctuations. This random field and its generator of realizations are constructed in the previous paragraph "Construction of random field $\left[\mathbf{G}_{0}\right]$ and its generator of realizations" of Section "Algebraic prior stochastic model for the case of anisotropic statistical fluctuations" (see Eq. (70)). The random field $\left\{\left[\mathbf{G}_{0}(\mathbf{x})\right], \mathbf{x} \in \Omega\right\}$ is defined on the probability space $(\Theta, \mathcal{T}, \mathcal{P})$, is indexed by $\mathbb{R}^{d}$, with values in $\mathbb{M}_{n}^{+}(\mathbb{R})$, is nonGaussian, homogeneous, second-order, and mean-square continuous on $\mathbb{R}^{d}$. 
- For all $\mathbf{x}$ in $\mathbb{R}^{d}$, the mean value of random matrix $\left[\mathbf{G}_{0}(\mathbf{x})\right]$ is matrix $\left[I_{n}\right]$ (see Eq. (71)).

- The level of the anisotropic statistical fluctuations is controlled by the dispersion parameter $\delta$ (independent of $\mathbf{x}$ ) such that $0<\delta<\sqrt{(n+1) /(n+5)}$ (see Eq. (72)).

- The hyperparameter $\mathbf{s}_{G_{0}}$ of random field $\left[\mathbf{G}_{0}\right]$ is constituted of the dispersion parameter $\delta$ and of the spatial-correlation lengths $\left\{L_{1}^{j k}, \ldots, L_{d}^{j k}\right\}_{1 \leq j \leq k \leq n}$ that are positive real numbers (see Eq. (63)).

$\left.\mathbb{R}^{d}\right\}$.

- Statistical fluctuations in the given symmetry class described by $\{[\boldsymbol{A}(\boldsymbol{x})], \boldsymbol{x} \in$

The random field $\left\{[\mathbf{A}(\mathbf{x})], \mathbf{x} \in \mathbb{R}^{d}\right\}$ models the statistical fluctuations belonging to the given symmetry class $\mathbb{M}_{n}^{\text {sym }}(\mathbb{R})$. This random field, defined on the probability space $\left(\Theta^{\prime}, \mathcal{T}^{\prime}, \mathcal{P}^{\prime}\right)$, is statistically independent of random field $\left\{\left[\mathbf{G}_{0}(\mathbf{x})\right], \mathbf{x} \in \mathbb{R}^{d}\right\}$, is indexed by $\mathbb{R}^{d}$, with values in $\mathbb{M}_{n}^{\text {sym }}(\mathbb{R}) \subset \mathbb{M}_{n}^{+}(\mathbb{R})$, non-Gaussian, homogeneous, second-order, and mean-square continuous on $\mathbb{R}^{d}$. In Eq. (88), for all $\mathbf{x}$ fixed in $\mathbb{R}^{d}$, the random matrix $[\mathbf{A}(\mathbf{x})]^{1 / 2}$ is the square root of random matrix $[\mathbf{A}(\mathbf{x})]$, and due to Eq. (79), is with values in $\mathbb{M}_{n}^{\text {sym }}(\mathbb{R}) \subset \mathbb{M}_{n}^{+}(\mathbb{R})$.

- For all $\mathbf{x}$ in $\mathbb{R}^{d}$, the mean value of random matrix $[\mathbf{A}(\mathbf{x})]$ is the matrix $[\underline{A}]$ (independent of $\mathbf{x}$ and defined by Eq. (84)) such that

$$
E\{[\mathbf{A}(\mathbf{x})]\}=[\underline{A}] \in \mathbb{M}_{n}^{\text {sym }}(\mathbb{R}) \subset \mathbb{M}_{n}^{+}(\mathbb{R}) .
$$

- In order that, for all $\mathbf{x}$ in $\mathbb{R}^{d}$, the zero matrix be a repulsive value for random matrix $[\mathbf{A}(\mathbf{x})]$, the following constraint is introduced,

$$
E\{\log (\operatorname{det}[\mathbf{A}(\mathbf{x})])\}=c_{A} \quad, \quad\left|c_{A}\right|<+\infty,
$$

in which real constant $c_{A}$ is independent of $\mathbf{x}$.

- The level of the statistical fluctuations belonging to the given symmetry class is controlled by the dispersion parameter $\delta_{A}$ (independent of $\mathbf{x}$ ) defined by

$$
\delta_{A}=\sqrt{\frac{E\left\{\|\mathbf{A}(\mathbf{x})-\underline{A}\|_{F}^{2}\right\}}{\|\underline{A}\|_{F}^{2}}}=\sqrt{\frac{E\left\{\|\mathbf{A}(\mathbf{x})\|_{F}^{2}\right\}}{\|\underline{A}\|_{F}^{2}}-1} .
$$

- Due to the statistical independence of $[\mathbf{A}(\mathbf{x})]$ and $\left[\mathbf{G}_{0}(\mathbf{x})\right]$, taking the mathematical expectation of the two members of Eq. (88), and from Eqs. (83) and (85), it can be deduced that, for all $\mathbf{x}$ in $\Omega$,

$$
E\left\{\left[\mathbf{K}^{\mathrm{APSM}}(\mathbf{x})\right]\right\}=[\underline{K}] \quad, \quad\left[\mathbf{K}^{\mathrm{APSM}}(\mathbf{x})\right]-\left[C_{\ell}\right]>0 \quad \text { a.s. }
$$


Remarks concerning the control of the statistical fluctuations and the limit cases.

Anisotropic statistical fluctuations going to zero $(\delta \rightarrow 0)$.

For a given symmetry class with $N<21$, if the level of anisotropic statistical fluctuations goes to zero, i.e., if $\delta \rightarrow 0$, which implies that, for all $\mathbf{x}$ in $\mathbb{R}^{d}$, random matrix $\left[\mathbf{G}_{0}(\mathbf{x})\right]$ goes to $\left[I_{n}\right]$ (in probability distribution), and implies that $[\underline{A}]$ goes to $[\underline{C}]$ and thus $[\underline{S}]$ goes to $\left[I_{n}\right]$, then Eq. (86) shows that $\left[\mathbf{K}^{\mathrm{APSM}}(\mathbf{x})\right]-\left[C_{\ell}\right]$ goes to $[\mathbf{A}(\mathbf{x})]$ (in probability distribution), which is a random matrix with values in $\mathbb{M}_{n}^{\text {sym }}(\mathbb{R})$. Consequently, if there are no anisotropic statistical fluctuations $(\delta=0)$, then Eq. (88) becomes

$$
\left[\mathbf{K}^{\mathrm{APSM}}(\mathbf{x})\right]=\left[C_{\ell}\right]+[\mathbf{A}(\mathbf{x})] \quad, \quad \forall \mathbf{x} \in \Omega,
$$

and $\left\{\left[\mathbf{K}^{\mathrm{APSM}}(\mathbf{x})\right], \mathbf{x} \in \Omega\right\}$ is a random field indexed by $\Omega$ with values in $\mathbb{M}_{n}^{\mathrm{sym}}(\mathbb{R})$.

- Statistical fluctuations in the symmetry class going to zero $\left(\delta_{A} \rightarrow 0\right)$.

If the given symmetry class is anisotropic $(N=21)$ and if $\delta_{A} \rightarrow 0$, then $[\underline{A}]$ goes to the mean matrix $[\underline{C}]$ and $[\underline{S}]$ goes to $\left[I_{n}\right]$, and Eq. (88) shows that $\left[\mathbf{K}^{\text {APSM }}(\mathbf{x})\right]-$ $\left[C_{\ell}\right]$ goes to $[\underline{C}]^{1 / 2}\left[\mathbf{G}_{0}(\mathbf{x})\right][\underline{C}]^{1 / 2}$ (in probability distribution), which is a random matrix with values in $\mathbb{M}_{n}^{+}(\mathbb{R})$. Consequently, if there are no statistical fluctuations in the symmetry class $\left(\delta_{A}=0\right)$, then Eq. (86) becomes

$$
\left[\mathbf{K}^{\mathrm{APSM}}(\mathbf{x})\right]=\left[C_{\ell}\right]+[\underline{C}]^{1 / 2}\left[\mathbf{G}_{0}(\mathbf{x})\right][\underline{C}]^{1 / 2} \quad, \quad \forall \mathbf{x} \in \Omega,
$$

which is Eq. (58).

Parameterization of random field $\left\{[\mathbf{A}(\mathbf{x})], \mathbf{x} \in \mathbb{R}^{d}\right\}$.

Random field $\left\{[\mathbf{A}(\mathbf{x})], \mathbf{x} \in \mathbb{R}^{d}\right\}$, with values in $\mathbb{M}_{n}^{\text {sym }}(\mathbb{R}) \subset \mathbb{M}_{n}^{+}(\mathbb{R})$, is written as

$$
[\mathbf{A}(\mathbf{x})]=[\underline{A}]^{1 / 2}[\mathbf{N}(\mathbf{x})][\underline{A}]^{1 / 2} \quad, \quad \forall \mathbf{x} \in \mathbb{R}^{d},
$$

in which $\left\{[\mathbf{N}(\mathbf{x})], \mathbf{x} \in \mathbb{R}^{d}\right\}$ is the random field indexed by $\mathbb{R}^{d}$ with values in $\mathbb{M}_{n}^{\operatorname{sym}}(\mathbb{R})$,

$$
[\mathbf{N}(\mathbf{x})]=\exp _{\mathbb{M}}\left(\sum_{j=1}^{N} Y_{j}(\mathbf{x})\left[E_{j}^{\mathrm{sym}}\right]\right) \quad, \quad \forall \mathbf{x} \in \mathbb{R}^{d}
$$


in which $\exp _{\mathbb{M}}$ denotes the exponential of the symmetric real matrices, where $\mathbf{Y}(\mathbf{x})=\left(Y_{1}(\mathbf{x}), \ldots, Y_{N}(\mathbf{x})\right)$, and where $\left\{\mathbf{Y}(\mathbf{x}), \mathbf{x} \in \mathbb{R}^{d}\right\}$ is a non-Gaussian random field defined on $\left(\Theta^{\prime}, \mathcal{T}^{\prime}, \mathcal{P}^{\prime}\right)$, indexed by $\mathbb{R}^{d}$ with values in $\mathbb{R}^{N}$, homogeneous, second-order, mean-square continuous on $\mathbb{R}^{d}$. Using the change of representation defined by Eqs. (81) and (82), random matrix $[\mathbf{N}(\mathbf{x})]$ defined by Eq. (96) can be rewritten as

$$
[\mathbf{N}(\mathbf{x})]=\sum_{j=1}^{N} m_{j}(\mathbf{Y}(\mathbf{x}))\left[E_{j}^{\mathrm{sym}}\right] .
$$

- Remark concerning the set of the values of random matrix $[\mathbf{A}(\mathbf{x})]$.

For all $\mathbf{x}$ fixed in $\mathbb{R}^{d},[\mathbf{N}(\mathbf{x})]$ is a random matrix with values in $\mathbb{M}_{n}^{\text {sym }}(\mathbb{R}$ ) (see Eq. (96)) and $[\underline{A}]$ is in $\mathbb{M}_{n}^{\text {sym }}(\mathbb{R})$ (see Eq. (89)). From Eqs. (79) and (95), it can be deduced that random matrix $[\mathbf{A}(\mathbf{x})]$ is in $\mathbb{M}_{n}^{\operatorname{sym}}(\mathbb{R}) \subset \mathbb{M}_{n}^{+}(\mathbb{R})$.

Available information for random matrix $[\mathbf{N}(\mathbf{x})]$.

For all $\mathbf{x}$ fixed in $\mathbb{R}^{d}$, substituting the representation of $[\mathbf{A}(\mathbf{x})]$ defined by Eq. (95) into Eqs. (89) and (90), yields the following available information for random matrix $[\mathbf{N}(\mathbf{x})]$,

$$
\begin{gathered}
E\{[\mathbf{N}(\mathbf{x})]\}=\left[I_{n}\right], \\
E\{\log (\operatorname{det}[\mathbf{N}(\mathbf{x})])\}=c_{N} \quad, \quad\left|c_{N}\right|<+\infty,
\end{gathered}
$$

in which real constant $c_{N}$ is independent of $\mathbf{x}$.

Available information for random matrix $\mathbf{Y}(\mathbf{x})$.

Substituting the representation of $[\mathbf{N}(\mathbf{x})]$ defined by Eq. (96) into the constraint defined by Eq. (99) yields the following constraint for $\mathbf{Y}(\mathbf{x})$,

$$
E\left\{\sum_{j=1}^{N} Y_{j}(\mathbf{x}) \operatorname{tr}\left[E_{j}^{\mathrm{sym}}\right]\right\}=c_{N} \quad, \quad\left|c_{N}\right|<+\infty \quad, \quad \forall \mathbf{x} \in \mathbb{R}^{d} .
$$

Substituting the representation of $[\mathbf{N}(\mathbf{x})]$ defined by Eq. (97) into the constraint defined by Eq. (98), yields $E\left\{\sum_{j=1}^{N} m_{j}(\mathbf{Y}(\mathbf{x}))\left[E_{j}^{\text {sym }}\right]\right\}=\left[I_{n}\right]$. Performing the projection of this equation on the basis $\left\{\left[E_{k}^{\text {sym }}\right], k=1, \ldots, N\right\}$ yields (similarly to Eq. (82)), $[\mathcal{E}] E\{\mathbf{m}(\mathbf{Y}(\mathbf{x}))\}=\mathcal{I}$ in which $\mathcal{I}=\left(\mathcal{I}_{1}, \ldots, \mathcal{I}_{N}\right)$ is the vector in $\mathbb{R}^{N}$ such that $\left.\mathcal{I}_{k}=\ll\left[I_{n}\right],\left[E_{k}^{\text {sym }}\right]\right) \gg$. The constraint on $[\mathbf{N}(\mathbf{x})]$ defined by Eq. (98) is transferred in the following constraint on $\mathbf{Y}(\mathbf{x})$,

$$
E\{\mathbf{m}(\mathbf{Y}(\mathbf{x}))\}=[\mathcal{E}]^{-1} \mathcal{I} \quad \text { on } \quad \mathbb{R}^{N}
$$


The constraints defined by Eqs. (100) and (101) are globally rewritten as

$$
E\{\mathbf{g}(\mathbf{Y}(\mathbf{x}))\}=\mathbf{f} \quad \text { on } \quad \mathbb{R}^{1+N},
$$

in which

- $\mathbf{y} \mapsto \mathbf{g}(\mathbf{y})=\left(g_{1}(\mathbf{y}), \ldots, g_{1+N}(\mathbf{y})\right)$ is the mapping from $\mathbb{R}^{N}$ into $\mathbb{R}^{1+N}$ such that $g_{1}(\mathbf{y})=\sum_{j=1}^{N} y_{j} \operatorname{tr}\left[E_{j}^{\text {sym }}\right]$ and $g_{1+j}(\mathbf{y})=m_{j}(\mathbf{y})$ for $j=1, \ldots, N$.

- $\mathbf{f}=\left(f_{1}, \ldots, f_{1+N}\right)$ is the vector in $\mathbb{R}^{1+N}$ such that $f_{1}=c_{N}$ and $f_{1+j}=$ $\left\{[\mathcal{E}]^{-1} \mathcal{I}\right\}_{j}$ for $j=1, \ldots, N$.

Construction of the pdf for random vector $\mathbf{Y}(\mathbf{x})$ using the MaxEnt principle. For all $\mathbf{x}$ fixed in $\mathbb{R}^{d}$, the probability density function $\mathbf{y} \mapsto p_{\mathbf{Y}(\mathbf{x})}(\mathbf{y})$ from $\mathbb{R}^{N}$ into $\mathbb{R}^{+}$of the $\mathbb{R}^{N}$-valued random vector $\mathbf{Y}(\mathbf{x})$, is independent of $\mathbf{x}$ (Y is homogeneous). This pdf is constructed using the maximum entropy principle presented in Section "MaxEnt for Constructing the pdf of a Random Vector" of "Random Matrix Models and Nonparametric Method for Uncertainty Quantification" in part II of the present Handbook on Uncertainty Quantification, under the constraints defined by the normalization condition $\int_{\mathbb{R}^{N}} p_{\mathbf{Y}(\mathbf{x})}(\mathbf{y}) d \mathbf{y}=1$ and by Eq. (102). For all $\mathbf{y}$ in $\mathbb{R}^{N}$, the pdf is written as

$$
p_{\mathbf{Y}(\mathbf{x})}(\mathbf{y})=c_{0}\left(\boldsymbol{\lambda}^{\mathrm{sol}}\right) \exp \left(-<\boldsymbol{\lambda}^{\mathrm{sol}}, \mathbf{g}(\mathbf{y})>\right) \quad, \quad \forall \mathbf{y} \in \mathbb{R}^{N},
$$

in which $c_{0}(\boldsymbol{\lambda})$ is defined by

$$
c_{0}(\boldsymbol{\lambda})=\left\{\int_{\mathbb{R}^{N}} \exp (-<\boldsymbol{\lambda}, \mathbf{g}(\mathbf{y})>) d \mathbf{y}\right\}^{-1}, \quad \boldsymbol{\lambda} \in \mathbb{R}^{1+N},
$$

where the Lagrange multiplier $\boldsymbol{\lambda}^{\text {sol }}=\left(\lambda_{1}^{\text {sol }}, \ldots, \lambda_{1+N}^{\text {sol }}\right)$ belongs to an admissible set $\mathcal{C}_{\lambda} \subset \mathbb{R}^{1+N}$ and is calculated for satisfying Eq. (102) by using the efficient numerical method presented in "Numerical Calculation of the Lagrange Multipliers" with the MCMC generator presented in "Generator for Random Vector $\mathbf{Y}_{\lambda}$ and Estimation of the Mathematical Expectations in High Dimension" of "Random Matrix Models and Nonparametric Method for Uncertainty Quantification" in part II of the present Handbook on Uncertainty Quantification.

Remark. In pdf $p_{\mathbf{Y}(\mathbf{x})}(\mathbf{y})$ constructed with Eq. (103), the Lagrange multiplier $\boldsymbol{\lambda}^{\text {sol }}$ depends only on one real parameter that is $c_{N}$. Such a parameter has no physical meaning and must be expressed as a function, $\kappa$, of the coefficient of variation $\delta_{A}$ defined by Eq. (91), such that $c_{N}=\kappa\left(\delta_{A}\right)$. This means that the family of the pdf constructed with Eq. (103) is reparameterized as a function of the dispersion 
parmeter $\delta_{A}$ using $c_{N}=\kappa\left(\delta_{A}\right)$. An explicit expression of function $\kappa$ cannot be obtained and is constructed numerically in using Eq. (91) in which $E\left\{\|\mathbf{A}(\mathbf{x})\|_{F}^{2}\right\}=$ $\left.\sum_{j=1}^{N} \sum_{k=1}^{N} \ll\left[E_{j}^{\mathrm{sym}}\right][\underline{A}],[\underline{A}]\left[E_{k}^{\mathrm{sym}}\right]\right) \gg \int_{\mathbb{R}^{N}} m_{j}(\mathbf{y}) m_{k}(\mathbf{y}) p_{\mathbf{Y}(\mathbf{x})}(\mathbf{y}) d \mathbf{y}$.

Constructing a spatial-correlation structure for $\operatorname{random}$ field $\left\{\mathbf{Y}(\mathbf{x}), \mathbf{x} \in \mathbb{R}^{d}\right\}$ and its generator.

A spatial-correlation structure is introduced as proposed in [32] for the non-Gaussian second-order homogeneous random field $\left\{\mathbf{Y}(\mathbf{x}), \mathbf{x} \in \mathbb{R}^{d}\right\}$ with values in $\mathbb{R}^{N}$, for which its first-order marginal probability density function $\mathbf{y} \mapsto p_{\mathbf{Y}(\mathbf{x})}(\mathbf{y})$ (see Eq. (103)) is imposed. This pdf is independent of $\mathbf{x}$ and depends on dispersion parameter $\delta_{A}$. Such a spatial-correlation structure for random field $\{\mathbf{Y}(\mathbf{x}), \mathbf{x} \in$ $\left.\mathbb{R}^{d}\right\}$ is transferred to random field $\left\{\mathbf{A}(\mathbf{x}), \mathbf{x} \in \mathbb{R}^{d}\right\}$ thanks to the transformation defined by Eqs. (95) and (96), which is written, for all $\mathbf{x}$ in $\mathbb{R}^{d}$, as $[\mathbf{A}(\mathbf{x})]=$ $[\underline{A}]^{1 / 2} \exp _{\mathbb{M}}\left(\sum_{j=1}^{N} Y_{j}(\mathbf{x})\left[E_{j}^{\text {sym }}\right]\right)[\underline{A}]^{1 / 2}$.

- Introduction of a Gaussian random field $\left\{\mathbf{B}(\mathbf{x}), \mathbf{x} \in \mathbb{R}^{d}\right\}$ that defines the spatial-correlation structure.

(i) Let $\mathbf{B}=\left(B_{1}, \ldots, B_{N}\right)$ be a random field defined on the probability probability space $\left(\Theta^{\prime}, \mathcal{T}^{\prime}, \mathcal{P}^{\prime}\right)$, indexed by $\mathbb{R}^{d}$, with values in $\mathbb{R}^{N}$, such that the components $B_{1}, \ldots, B_{N}$ are $N$ independent real-valued second-order random fields that are Gaussian, homogeneous, centered, normalized and mean-square continuous. The continuous autocorrelation function $\boldsymbol{\zeta} \mapsto\left[R_{\mathbf{B}}(\boldsymbol{\zeta})\right]=E\left\{\mathbf{B}(\mathbf{x}+\boldsymbol{\zeta}) \mathbf{B}(\mathbf{x})^{T}\right\}$ from $\mathbb{R}^{d}$ into $\mathbb{M}_{N}(\mathbb{R})$ is thus diagonal,

$$
\left[R_{\mathbf{B}}(\boldsymbol{\zeta})\right]_{j k}=\delta_{j k} R_{j}(\boldsymbol{\zeta}) \quad, \quad\left[R_{\mathbf{B}}(\mathbf{0})\right]=\left[I_{N}\right]
$$

in which $\left.\boldsymbol{\zeta} \mapsto R_{j}(\boldsymbol{\zeta})\right]=E\left\{B_{j}(\mathbf{x}+\boldsymbol{\zeta}) B_{j}(\mathbf{x})\right\}$, from $\mathbb{R}^{d}$ into $\mathbb{R}$, is the autocorrelation function of the centered random field $\left\{B_{j}(\mathbf{x}), \mathbf{x} \in \mathbb{R}^{d}\right\}$. For all fixed $j$, since the second-order random field $\left\{B_{j}(\mathbf{x}), \mathbf{x} \in \mathbb{R}^{d}\right\}$ is Gaussian and centered, this random field is completely and uniquely defined by its autocorrelation function $R_{j}(\boldsymbol{\zeta})=E\left\{B_{j}(\mathbf{x}+\boldsymbol{\zeta}) B_{j}(\mathbf{x})\right\}$ defined for all $\boldsymbol{\zeta}=\left(\zeta_{1}, \ldots, \zeta_{d}\right)$ in $\mathbb{R}^{d}$ and such that $R_{j}(0)=1$. The spatial-correlation lengths $\mathbb{L}_{1}^{j}, \ldots, \mathbb{L}_{d}^{j}$ of random field $\left\{B_{j}(\mathbf{x}), \mathbf{x} \in \mathbb{R}^{d}\right\}$ are defined by

$$
\mathbb{L}_{\alpha}^{j}=\int_{0}^{+\infty}\left|R_{j}\left(0, \ldots, \zeta_{\alpha}, \ldots, 0\right)\right| d \zeta_{\alpha}
$$

In the parameterization of each autocorrelation function $R_{j}$, the parameters $\mathbb{L}_{1}^{j}, \ldots$, $\mathbb{L}_{d}^{j}$ are generally chosen as hyperparameters. 
Example of parameterization for autocorrelation function $R_{j}$. A minimal parameterization can be defined as $R_{j}(\boldsymbol{\zeta})=\rho_{1}^{j}\left(\zeta_{1}\right) \times \ldots \times \rho_{d}^{j}\left(\zeta_{d}\right)$ in which, for all $\alpha=1, \ldots, d, \rho_{\alpha}^{j}(0)=1$ and where, for $\zeta_{\alpha} \neq 0$,

$$
\rho_{\alpha}^{j}\left(\zeta_{\alpha}\right)=4\left(\mathbb{L}_{\alpha}^{j}\right)^{2} /\left(\pi^{2} \zeta_{\alpha}^{2}\right) \sin ^{2}\left(\pi \zeta_{\alpha} /\left(2 \mathbb{L}_{\alpha}^{j}\right)\right)
$$

in which $\mathbb{L}_{1}^{j}, \ldots, \mathbb{L}_{d}^{j}$ are positive real numbers. Each random field $B_{j}$ is meansquare continuous on $\mathbb{R}^{d}$ and its power spectral density function defined on $\mathbb{R}^{d}$ has a compact support, $\left[-\pi / \mathbb{L}_{1}^{j}, \pi / \mathbb{L}_{1}^{j}\right] \times \ldots \times\left[-\pi / \mathbb{L}_{1}^{j}, \pi / \mathbb{L}_{d}^{j}\right]$. The parameters, $\mathbb{L}_{1}^{j}, \ldots, \mathbb{L}_{d}^{j}$, represent the spatial-correlation lengths of the stochastic germ $\left\{B_{j}(\mathbf{x}), \mathbf{x} \in \mathbb{R}^{d}\right\}$.

(ii) For all countable ordered subsets $0 \leq r_{1}<\ldots<r_{k}<r_{k+1}<\ldots$ of $\mathbb{R}^{+}$, the sequence of random fields $\left\{\mathbf{B}^{r_{k} r_{k+1}}(\mathbf{x}), \mathbf{x} \in \mathbb{R}^{d}\right\}_{k \in \mathbb{N}}$

- are mutually independent random fields,

- are such that, $\forall k \in \mathbb{N},\left\{\mathbf{B}^{r_{k} r_{k+1}}(\mathbf{x}), \mathbf{x} \in \mathbb{R}^{d}\right\}$ is an independent copy of $\left\{\mathbf{B}(\mathbf{x}), \mathbf{x} \in \mathbb{R}^{d}\right\}$, which implies that $E\left\{\mathbf{B}^{r_{k} r_{k+1}}(\mathbf{x})\right\}=E\{\mathbf{B}(\mathbf{x})\}=\mathbf{0}$ and that

$$
E\left\{\mathbf{B}^{r_{k} r_{k+1}}(\mathbf{x})\left(\mathbf{B}^{r_{k} r_{k+1}}(\mathbf{x})\right)^{T}\right\}=E\left\{\mathbf{B}(\mathbf{x}) \mathbf{B}(\mathbf{x})^{T}\right\}=\left[R_{\mathbf{B}}(\mathbf{0})\right]=\left[I_{N}\right] .
$$

- Defining an $\mathbf{x}$-dependent family of normalized Wiener stochastic processes $\left\{\mathbf{W}_{\mathbf{x}}(r), r \geq 0\right\}$ containing the spatial-correlation structure.

Let $\left\{\mathbf{W}_{\mathbf{x}}(r), r \geq 0\right\}$ be the $\mathbf{x}$-dependent family of stochastic processes defined on probability space $\left(\Theta^{\prime}, \mathcal{T}^{\prime}, \mathcal{P}^{\prime}\right)$, indexed by $r \geq 0$, with values in $\mathbb{R}^{N}$, such that $\mathbf{W}_{\mathbf{x}}(0)=\mathbf{0}$ almost surely and, for all $\mathbf{x}$ fixed $\mathbb{R}^{d}$ and for all $0 \leq s<r<+\infty$, the increment $\Delta \mathbf{W}_{\mathbf{x}}^{s r}:=\mathbf{W}_{\mathbf{x}}(r)-\mathbf{W}_{\mathbf{x}}(s)$ is written as

$$
\Delta \mathbf{W}_{\mathbf{x}}^{s r}=\sqrt{r-s} \mathbf{B}^{s r}(\mathbf{x}) .
$$

From the properties of random field $\left\{\mathbf{B}(\mathbf{x}), \mathbf{x} \in \mathbb{R}^{d}\right\}$ and of the family of random fields $\left\{\mathbf{B}^{r_{k} r_{k+1}}(\mathbf{x}), \mathbf{x} \in \mathbb{R}^{d}\right\}_{k \in \mathbb{N}}$ for all countable ordered subsets $0 \leq r_{1}<\ldots<$ $r_{k}<r_{k+1}<\ldots$, it is deduced that, for all $\mathbf{x}$ fixed in $\mathbb{R}^{d}$,

(i) the components $W_{\mathbf{x}}^{(1)}, \ldots, W_{\mathbf{x}}^{(N)}$ of $\mathbf{W}_{\mathbf{x}}$ are mutually independent real-valued stochastic processes,

(ii) $\left\{\mathbf{W}_{\mathbf{x}}(r), r \geq 0\right\}$ is a stochastic process with independent increments,

(iii) For all $0 \leq s<r<+\infty$, the increment $\Delta \mathbf{W}_{\mathbf{x}}^{s r}=\mathbf{W}_{\mathbf{x}}(r)-\mathbf{W}_{\mathbf{x}}(s)$ is a $\mathbb{R}^{N}$-valued second-order random variable which is Gaussian, centered, and with a covariance matrix that is written as $\left[C_{\Delta \mathbf{W}_{\mathbf{x}}^{s r}}\right]=E\left\{\Delta \mathbf{W}_{\mathbf{x}}^{s r}\left(\Delta \mathbf{W}_{\mathbf{x}}^{s r}\right)^{T}\right\}=(r-$ s) $\left[I_{N}\right]$. 
(iv) Since $\mathbf{W}_{\mathbf{x}}(0)=\mathbf{0}$, and from (i), (ii), and (iii), it can be deduced that $\left\{\mathbf{W}_{\mathbf{x}}(r), r \geq\right.$ $0\}$ is a $\mathbb{R}^{N}$-valued normalized Wiener process.

- Constructing random field $\left\{\mathbf{Y}(\mathbf{x}), \mathbf{x} \in \mathbb{R}^{d}\right\}$ and its generator.

The construction of random field $\left\{\mathbf{Y}(\mathbf{x}), \mathbf{x} \in \mathbb{R}^{d}\right\}$ is carried out by introducing a family (indexed by $\mathbf{x}$ in $\mathbb{R}^{d}$ ) of Itô stochastic differential equations (ISDE),

- for which the Wiener process is the family $\left\{\mathbf{W}_{\mathbf{x}}(r), r \geq 0\right\}$ that contains the imposed spatial-correlation structure defined by Eq. (105),

- that admits the same unique invariant measure (independent of $\mathbf{x}$ ), which is defined by the pdf $p_{\mathbf{Y}(\mathbf{x})}$ given by Eqs. (103)-(104).

Taking into account Eq. (103), the potential $\mathbf{u} \mapsto \Phi(\mathbf{u})$, from $\mathbb{R}^{N}$ into $\mathbb{R}$, is defined by

$$
\Phi(\mathbf{u})=<\boldsymbol{\lambda}^{\mathrm{sol}}, \mathbf{g}(\mathbf{u})>.
$$

For all $\mathbf{x}$ fixed in $\mathbb{R}^{d}$, let $\left\{\left(\mathbf{U}_{\mathbf{x}}(r), \mathbf{V}_{\mathbf{x}}(r)\right), r \geq 0\right\}$ be the Markov stochastic process defined on the probability space $\left(\Theta^{\prime}, \mathcal{T}^{\prime}, \mathcal{P}^{\prime}\right)$, indexed by $r \geq 0$, with values in $\mathbb{R}^{N} \times \mathbb{R}^{N}$, satisfying, for all $r>0$, the following ISDE,

$$
\begin{gathered}
d \mathbf{U}_{\mathbf{x}}(r)=\mathbf{V}_{\mathbf{x}}(r) d r \\
d \mathbf{V}_{\mathbf{x}}(r)=-\nabla_{\mathbf{u}} \Phi\left(\mathbf{U}_{\mathbf{x}}(r)\right) d r-\frac{1}{2} f_{0} \mathbf{V}_{\mathbf{x}}(r) d r+\sqrt{f_{0}} d \mathbf{W}_{\mathbf{x}}(r),
\end{gathered}
$$

with the initial conditions,

$$
\mathbf{U}_{\mathbf{x}}(0)=\mathbf{u}_{0} \quad, \quad \mathbf{V}_{\mathbf{x}}(0)=\mathbf{v}_{0} \quad \text { a.s. },
$$

in which $\mathbf{u}_{0}$ and $\mathbf{v}_{0}$ are given vectors in $\mathbb{R}^{N}$ (that are generally taken as zero in the applications) and $f_{0}>0$ is a free parameter whose usefulness is explained below. From Eqs. (82) and (102), it can be deduced that function $\mathbf{u} \mapsto \Phi(\mathbf{u})$ : (i) is continuous on $\mathbb{R}^{N}$, (ii) is such that $\mathbf{u} \mapsto\left\|\nabla_{\mathbf{u}} \Phi(\mathbf{u})\right\|$ is a locally bounded function on $\mathbb{R}^{N}$ (i.e. is bounded on all compact sets in $\mathbb{R}^{N}$ ). In addition the Lagrange multiplier $\boldsymbol{\lambda}^{\text {sol }}$, which belongs to $\mathcal{C}_{\boldsymbol{\lambda}} \subset \mathbb{R}^{1+N}$, is such that

$$
\begin{gathered}
\inf _{\|\mathbf{u}\|>R} \Phi(\mathbf{u}) \rightarrow+\infty \quad \text { if } \quad R \rightarrow+\infty, \\
\inf _{\mathbf{u} \in \mathbb{R}^{n}} \Phi(\mathbf{u})=\Phi_{\min } \quad \text { with } \quad \Phi_{\min } \in \mathbb{R}, \\
\int_{\mathbb{R}^{n}}\left\|\nabla_{\mathbf{u}} \Phi(\mathbf{u})\right\| e^{-\Phi(\mathbf{u})} d \mathbf{u}<+\infty .
\end{gathered}
$$


Taking into account (i), (ii), and Eqs. (112) to (114), using Theorems 4 to 7 in pages 211 to 216 of Ref. [55] for which the Hamiltonian is taken as $\mathbb{H}(\mathbf{u}, \mathbf{v})=$ $\|\mathbf{v}\|^{2} / 2+\Phi(\mathbf{u})$, and using [17; 39] for the ergodic property, it can be deduced that the problem defined by Eqs. (109) to (111) admits a unique solution. For all $\mathbf{x}$ fixed in $\mathbb{R}^{d}$, this solution is a second-order diffusion stochastic process $\left\{\left(\mathbf{U}_{\mathbf{x}}(r), \mathbf{V}_{\mathbf{x}}(r)\right)\right.$, $r \geq 0\}$, which converges to a stationary and ergodic diffusion stochastic process $\left\{\left(\mathbf{U}_{\mathbf{x}}^{\text {st }}\left(r_{\text {st }}\right), \mathbf{V}_{\mathbf{x}}^{\text {st }}\left(r_{\text {st }}\right)\right), r_{\text {st }} \geq 0\right\}$, when $r$ goes to infinity, associated with the invariant probability measure $P_{\mathrm{st}}(d \mathbf{u}, d \mathbf{v})=\rho_{\mathrm{st}}(\mathbf{u}, \mathbf{v}) d \mathbf{u} d \mathbf{v}$ (that is independent of $\mathbf{x}$ ). The probability density function $(\mathbf{u}, \mathbf{v}) \mapsto \rho_{\mathrm{st}}(\mathbf{u}, \mathbf{v})$ on $\mathbb{R}^{N} \times \mathbb{R}^{N}$ is the unique solution of the steady-state Fokker-Planck equation associated with Eqs. (109)-(110), and is written (see pp. 120 to 123 in [55]), as

$$
\rho_{\mathrm{st}}(\mathbf{u}, \mathbf{v})=\mathbb{C}_{N} \exp \left\{-\frac{1}{2}\|\mathbf{v}\|^{2}-\Phi(\mathbf{u})\right\}
$$

in which $\mathbb{C}_{N}$ is the constant of normalization. Equations (103), (108), and (115) yield

$$
p_{\mathbf{Y}(\mathbf{x})}(\mathbf{y})=\int_{\mathbb{R}^{N}} \rho_{\mathrm{st}}(\mathbf{y}, \mathbf{v}) d \mathbf{v} \quad, \quad \forall \mathbf{y} \in \mathbb{R}^{N} .
$$

Random variable $\mathbf{Y}(\mathbf{x})$ (for which the pdf $p_{\mathbf{Y}(\mathbf{x})}$ is defined by Eq. (103)) can then be written, for all fixed positive value of $r_{\mathrm{st}}$, as

$$
\mathbf{Y}(\mathbf{x})=\mathbf{U}_{\mathbf{x}}^{\text {st }}\left(r_{\mathrm{st}}\right)=\lim _{r \rightarrow+\infty} \mathbf{U}_{\mathbf{x}}(r) \quad \text { in probability distribution } .
$$

The free parameter $f_{0}>0$ introduced in Eq. (110), allows a dissipation term to be introduced in the nonlinear second-order dynamical system (formulated in the Hamiltonian form with an additional dissipative term) for obtaining more rapidly the asymptotic behavior corresponding to the stationary and ergodic solution associated with the invariant measure. Using Eq. (117) and the ergodic property of stationary stochastic process $\mathbf{U}_{\mathbf{x}}^{\text {st }}$, it should be noted that, if $w$ is any mapping from $\mathbb{R}^{N}$ into an Euclidean space such that $E\{w(\mathbf{Y}(\mathbf{x}))\}=\int_{\mathbb{R}^{N}} w(\mathbf{y}) p_{\mathbf{Y}(\mathbf{x})} d \mathbf{y}$ is finite, then

$$
E\{w(\mathbf{Y}(\mathbf{x}))\}=\lim _{R \rightarrow+\infty} \frac{1}{R} \int_{0}^{R} w\left(\mathbf{U}_{\mathbf{x}}\left(r, \theta^{\prime}\right)\right) d r,
$$

in which, for $\theta^{\prime} \in \Theta^{\prime}, \mathbf{U}_{\mathbf{x}}\left(\cdot, \theta^{\prime}\right)$ is any realization of $\mathbf{U}_{\mathbf{x}}$.

\section{Discretization scheme of the family of ISDE.}

A discretization scheme must be used for numerically solving Eqs. (109) to (111). For general surveys on discretization schemes for ISDE, we refer the reader to [40; 
70] (among others). The present case, related to a Hamiltonian dynamical system, has also been analyzed using an implicit Euler scheme in [71]. Hereinafter, we present the Störmer-Verlet scheme (see [32;34]), which is an efficient scheme that preserves energy for nondissipative Hamiltonian dynamical systems (see [35] for reviews about this scheme in the deterministic case, and see [6] and the references therein for the stochastic case).

Let $\mu \geq 1$ be an integer. For all $\mathbf{x}$ in $\mathbb{R}^{d}$, the ISDE defined by Eqs. (109) to (111) is solved on the finite interval $[0,(\mu-1) \Delta r]$, in which $\Delta r$ is the sampling step of the continuous index parameter $r$. The integration scheme is based on the use of the $\mu$ sampling points $r_{k}=(k-1) \Delta r$ for $k=1, \ldots, \mu$, and the following notations are used: $\mathbf{U}_{\mathbf{x}}^{k}=\mathbf{U}_{\mathbf{x}}\left(r_{k}\right), \mathbf{V}_{\mathbf{x}}^{k}=\mathbf{V}_{\mathbf{x}}\left(r_{k}\right)$, and $\left.\mathbf{W}_{\mathbf{x}}^{k}=\mathbf{W}_{\mathbf{x}}\left(r_{k}\right)\right)$, with $\mathbf{U}_{\mathbf{x}}^{1}=$ $\mathbf{u}_{0}, \mathbf{V}_{\mathbf{x}}^{1}=\mathbf{v}_{0}$, and $\mathbf{W}_{\mathbf{x}}^{1}=\mathbf{W}_{\mathbf{x}}(0)=\mathbf{0}$. From Eq. (107) and for $k=1, \ldots, \mu-1$, the increment $\Delta \mathbf{W}_{\mathbf{x}}^{k+1}=\mathbf{W}_{\mathbf{x}}^{k+1}-\mathbf{W}_{\mathbf{x}}^{k}$ is written as

$$
\Delta \mathbf{W}_{\mathbf{x}}^{k+1}=\sqrt{\Delta r} \mathbf{B}^{k+1}(\mathbf{x}) \quad, \quad \forall \mathbf{x} \in \mathbb{R}^{d}
$$

in which the $\mu-1$ random fields $\left\{\mathbf{B}^{k+1}(\mathbf{x}), \mathbf{x} \in \mathbb{R}^{d}\right\}_{k=1, \ldots, \mu-1}$ are independent copies of random field $\left\{\mathbf{B}(\mathbf{x}), \mathbf{x} \in \mathbb{R}^{d}\right\}$. For $k=1, \ldots, \mu-1$, the Störmer-Verlet scheme is written as

$$
\begin{gathered}
\mathbf{U}_{\mathbf{x}}^{k+\frac{1}{2}}=\mathbf{U}_{\mathbf{x}}^{k}+\frac{\Delta r}{2} \mathbf{V}_{\mathbf{x}}^{k} \\
\mathbf{V}_{\mathbf{x}}^{k+1}=\frac{1-b}{1+b} \mathbf{V}_{\mathbf{x}}^{k}+\frac{\Delta r}{1+b} \mathbf{L}_{\mathbf{x}}^{k+\frac{1}{2}}+\frac{\sqrt{f_{0}}}{1+b} \Delta \mathbf{W}_{\mathbf{x}}^{k+1}, \\
\mathbf{U}_{\mathbf{x}}^{k+1}=\mathbf{U}_{\mathbf{x}}^{k+\frac{1}{2}}+\frac{\Delta r}{2} \mathbf{V}_{\mathbf{x}}^{k+1}
\end{gathered}
$$

where $b=f_{0} \Delta r / 4$, and where $\mathbf{L}_{\mathbf{x}}^{k+\frac{1}{2}}$ is the $\mathbb{R}^{N}$-valued random variable such that $\mathbf{L}_{\mathbf{x}}^{k+\frac{1}{2}}=-\left\{\nabla_{\mathbf{u}} \Phi(\mathbf{u})\right\}_{\mathbf{u}=\mathbf{U}_{\mathbf{x}}^{k+\frac{1}{2}}}$. For a given realization $\theta^{\prime}$ in $\Theta^{\prime}$, the sequence $\left\{\mathbf{U}_{\mathbf{x}}^{k}\left(\theta^{\prime}\right), k=1, \ldots, \mu\right\}$ is constructed using Eqs. (120) to (122). The discretization of Eq. (118) yields the following estimation of the mathematical expectation,

$$
E\{w(\mathbf{Y}(\mathbf{x}))\}=\lim _{\mu \rightarrow+\infty} \widehat{w}_{\mu}(\mathbf{x}) \quad, \quad \widehat{w}_{\mu}(\mathbf{x})=\frac{1}{\mu-\mu_{0}+1} \sum_{k=\mu_{0}}^{\mu} w\left(\mathbf{U}_{\mathbf{x}}^{k}\left(\theta^{\prime}\right)\right),
$$

in which, for $f_{0}$ fixed, the integer $\mu_{0}>1$ is chosen to remove the transient part of the response induced by the initial condition. For details concerning the optimal choice of the numerical parameters, such as $\mu_{0}, \mu, f_{0}, \Delta_{r}, \mathbf{u}_{0}$, and $\mathbf{v}_{0}$, we refer the 
reader to $[59 ; 33 ; 34]$.

\section{Definition of the hyperparameter $\mathbf{s}$.}

The hyperparameter parameter $\mathbf{s} \in \mathcal{C}_{s} \subset \mathbb{R}^{N_{s}}$ of the algebraic prior stochastic model $\left\{\left[\mathbf{K}^{\mathrm{APSM}}(\mathbf{x} ; \mathbf{s})\right], \mathbf{x} \in \Omega\right\}$, which has been constructed for the dominant statistical fluctuations belonging to a given symmetry class of dimension $n$, with some anisotropic statistical fluctuations, are constituted of the quantities summarized hereinafter:

- the reshaping of $\left[C_{\ell}\right] \in \mathbb{M}_{n}^{+}(\mathbb{R})$ (the lower bound) and $[\underline{K}] \in \mathbb{M}_{n}^{+}(\mathbb{R})$ (the mean value),

- for the control of the anisotropic statistical fluctuations (modeled by random field $\left[\mathbf{G}_{0}\right]$ ), the $d n(n+1) / 2$ positive real numbers, $\left\{L_{1}^{j k}, \ldots, L_{d}^{j k}\right\}_{1 \leq j \leq k \leq n}$ (the spatial-correlation lengths, for the parameterization given in the example), and $\delta$ (the dispersion) such that $0<\delta<\sqrt{(n+1) /(n+5)}$,

- for the control of the statistical fluctuations belonging to a symmetry class (modeled by random field $[\mathbf{A}]$ ), the $d N$ positive real numbers, $\left\{\mathbb{L}_{1}^{j}, \ldots, \mathbb{L}_{d}^{j}\right\}_{1 \leq j \leq N}$ (the spatial-correlation lengths, for the parameterization given in the example), and $\delta_{A}$ (the dispersion) such that $0<\delta_{A}$.

\section{Key Research Findings, and Applications}

Additional ingredients for statistical reduced models, symmetry properties and generators for high-stochastic dimension

- Karhunen-Loève's expansion revisited for vector-valued random fields and identification from a set of realizations: scaling [50], a posteriori error and optimal reduced basis [51].

- Construction of a basis adaptation in homogeneous chaos spaces [73].

- ISDE-based generator for a class of non-gaussian vector-valued random fields in uncertainty quantification $[32 ; 34]$.

- Random elasticity tensors of materials exhibiting symmetry properties [29; $30 ; 32]$ and stochastic boundedness constraints $[11 ; 28 ; 30]$.

- Random fields representations and robust algorithms for the identification of polynomial chaos representations in high dimension from a set of realizations $[62 ; 64 ; 49 ; 48 ; 51 ; 66]$.

\section{Tensor-valued random fields and continuum mechanics of heterogenous ma- terials}

- Composites reinforced with fibers with experimental identification [26; 27]. 
- Polycrystalline microstructures [28].

- Porous materials with anisotropic permeability tensor random field [31], and with interphases [33].

- Human cortical bone with mechanical alterations in ultrasonic range [16].

\section{Conclusions}

A complete advanced methodology and the associated tools have been presented for solving the challenging statistical inverse problem related to the experimental identification of a non-Gaussian matrix-valued random field that is the model parameter of a boundary value problem, using some partial and limited experimental data related to a model observation. Many applications and validation of this methodology can be found in the given references.

\section{References}

[1] Absil PA, Mahony R, and Sepulchre R (2000) Optimization Algorithms on Matrix Manifolds. Princeton University Press, Princeton.

[2] Arnst M, Ghanem R, and Soize C (2010) Identification of Bayesian posteriors for coefficients of chaos expansions. J. Comput. Physics 229(9): 31343154.

[3] Batou A, Soize C (2013) Stochastic modeling and identification of an uncertain computational dynamical model with random fields properties and model uncertainties. Archive of Applied Mechanics 83(6): 831-848.

[4] Batou A, Soize C (2013) Calculation of Lagrange multipliers in the construction of maximum entropy distributions in high stochastic dimension. SIAM/ASA J. Uncertainty Quantification 1(1): 431-451.

[5] Blatman G, Sudret B (2011) Adaptive sparse polynomial chaos expansion based on least angle regression. J. Comput. Physics 230(6): 2345-2367.

[6] Burrage K, Lenane I, and Lythe G (2007) Numerical methods for secondorder stochastic differential equations. SIAM J. Sci. Comput. 29: 245-264.

[7] Cameron RH, Martin WT (1947) The orthogonal development of non-linear functionals in series of Fourier-Hermite functionals. Ann. Math., Second Series 48(2): 385-392. 
[8] Carlin BP and Louis TA (2009) Bayesian Methods for Data Analysis. Third Edition, Chapman \& Hall / CRC Press, Boca Raton.

[9] Congdon P (2007) Bayesian Statistical Modelling. Second Edition, John Wiley \& Sons, Chichester.

[10] Das S, Ghanem R, and Spall JC (2008) Asymptotic sampling distribution for polynomial chaos representation from data: a maximum entropy and fisher information approach. SIAM J. Sci. Comput. 30(5): 2207-2234.

[11] Das S, Ghanem R (2009) A bounded random matrix approach for stochastic upscaling. Multiscale Model. Simul. 8(1): 296-325.

[12] Das S, Ghanem R, and Finette S (2009) Polynomial chaos representation of spatio-temporal random field from experimental measurements. J. Comput. Phys. 228: 8726-8751.

[13] Debusschere BJ, Najm HN, Pebay PP, Knio OM, Ghanem R, and Le Maître O (2004) Numerical challenges in the use of polynomial chaos representations for stochastic processes. SIAM J. Sci. Comput. 26(2): 698-719.

[14] Desceliers C, Ghanem R, Soize C (2006) Maximum likelihood estimation of stochastic chaos representations from experimental data. Int. J. Numer. Methods Eng. 66(6): 978-1001.

[15] Desceliers C, Soize C, Ghanem R (2007) Identification of chaos representations of elastic properties of random media using experimental vibration tests. Comput. Mech. 39(6): 831-838.

[16] Desceliers C, Soize C, Naili S, and Haiat G (2012) Probabilistic model of the human cortical bone with mechanical alterations in ultrasonic range. Mech. Syst. Signal Pr. 32: 170-177.

[17] Doob JL (1990) Stochastic Processes. John Wiley \& Sons, New York.

[18] Doostan A, Ghanem R, and Red-Horse J (2007) Stochastic model reduction for chaos representations. Comput. Methods Appl. Mech. Eng. 196(37-40): 3951-3966.

[19] Edelman A, Arias TA, and Smith ST (1998) The geometry of algorithms with orthogonality constraints. SIAM J. Matrix Anal. A., 20(2): 303-353. 
[20] Ernst OG, Mugler A, Starkloff HJ, and Ullmann E (2012) On the convergence of generalized polynomial chaos expansions. ESAIM - Math. Model. Num. 46(2): 317-339.

[21] Ghanem R and Spanos PD (1991) Stochastic Finite Elements: a Spectral Approach. Springer-Verlag, New York. See also the revised edition (2003), Dover Publications, New York.

[22] Ghanem R, Dham S (1998) Stochastic finite element analysis for multiphase flow in heterogeneous porous media. Transp. Porous Media 32: 239-262.

[23] Ghanem R, Doostan R (2006) Characterization of stochastic system parameters from experimental data: A bayesian inference approach. J. Comput. Physics 217(1): 63-81.

[24] Ghanem R, Doostan R, and Red-Horse J (2008) A probability construction of model validation. Comput. Methods Appl. Mech. Eng. 197(29-32): 25852595 .

[25] Ghosh D, Ghanem R (2008) Stochastic convergence acceleration through basis enrichment of polynomial chaos expansions. Int. J. Numer. Methods Eng. 73(2): 162-184.

[26] Guilleminot J, Soize C, Kondo D, and Binetruy C (2008) Theoretical framework and experimental procedure for modelling volume fraction stochastic fluctuations in fiber reinforced composites. Int. J. Solids Struct. 45(21): 5567-5583.

[27] Guilleminot J, Soize C, and Kondo D (2009) Mesoscale probabilistic models for the elasticity tensor of fiber reinforced composites: experimental identification and numerical aspects. Mech. Mater. 41(12): 1309-1322.

[28] Guilleminot J, Noshadravan A, Soize C, and Ghanem RG (2011) A probabilistic model for bounded elasticity tensor random fields with application to polycrystalline microstructures. Comput. Methods Appl. Mech. Eng. 200:16371648.

[29] Guilleminot J, Soize C (2011) Non-Gaussian positive-definite matrix-valued random fields with constrained eigenvalues: application to random elasticity tensors with uncertain material symmetries. Int. J. Numer. Methods Eng. 88(11): 1128-1151. 
[30] Guilleminot J, Soize C (2012) Probabilistic modeling of apparent tensors in elastostatics: a MaxEnt approach under material symmetry and stochastic boundedness constraints. Probab. Eng. Mech. 28: 118-124.

[31] Guilleminot J, Soize C, and Ghanem R (2012) Stochastic representation for anisotropic permeability tensor random fields. Int. J. Numer. Anal. Met. Geom. 36(13): 1592-1608.

[32] Guilleminot J, Soize C (2013) Stochastic model and generator for random fields with symmetry properties: application to the mesoscopic modeling of elastic random media. Multiscale Model. Simul. (A SIAM Interdisciplinary Journal) 11(3): 840-870.

[33] Guilleminot J, Le TT, Soize C (2013) Stochastic framework for modeling the linear apparent behavior of complex materials: application to random porous materials with interphases. Acta Mech. Sinica 29(6): 773-782.

[34] Guilleminot J, Soize C (2014) Itô SDE-based generator for a class of nongaussian vector-valued random fields in uncertainty quantification. SIAM J. Sci. Comput. 36(6): A2763-A2786.

[35] Hairer E, Lubich C, and G. Wanner G (2002) Geometric Numerical Integration. Structure-Preserving Algorithms for Ordinary Differential Equations. Springer-Verlag, Heidelberg.

[36] Isakov V (2006) Inverse Problems for Partial Differential Equations. Springer-Verlag, New York.

[37] Jaynes ET (1957) Information theory and statistical mechanics. Physical Review 106(4): 620-630 and 108(2): 171-190.

[38] Kaipio J, Somersalo E (2005) Statistical ans Computational Inverse Problems. Springer-Verlag, New York.

[39] Khasminskii R (2012) Stochastic Stability of Differential Equations, 2nd edition. Heidelberg, Springer.

[40] Kloeden PE, Platen E (1992) Numerical Solution of Stochastic Differentials Equations. Springer-Verlag, Heidelberg.

[41] Krée P and Soize C (1986) Mathematics of Random Phenomena. Reidel, Dordrecht. 
[42] Le Maitre OP, Knio OM, Najm HN (2004) Uncertainty propagation using Wiener-Haar expansions. J. Comput. Phys. 197(1): 28-57.

[43] Le Maître OP and Knio OM (2010) Spectral Methods for Uncertainty Quantification with Applications to Computational Fluid Dynamics. Springer, Heidelberg.

[44] Lucor D, Su CH, and Karniadakis GE (2004) Generalized polynomial chaos and random oscillators. Int. J. Numer. Methods Eng. 60(3): 571-596.

[45] Marzouk YM, Najm HN (2009) Dimensionality reduction and polynomial chaos acceleration of Bayesian inference in inverse problems. J. Comput. Physics 228(6): 1862-1902.

[46] Najm HH (2009) Uncertainty quantification and polynomial chaos techniques in computational fluid dynamics. Annu. Rev. Fluid Mech. 41: 35-52.

[47] Nouy A (2010) Proper Generalized Decomposition and separated representations for the numerical solution of high dimensional stochastic problems. Arch. Comput. Methods Eng. 16(3): 403-434.

[48] Nouy A, Soize C (2014) Random fields representations for stochastic elliptic boundary value problems and statistical inverse problems. Euro. Jnl of Applied Mathematics 25(3): 339-373.

[49] Perrin G, Soize C, Duhamel D, and Funfschilling C (2012) Identification of polynomial chaos representations in high dimension from a set of realizations. SIAM J. Sci. Comput. 34(6): A2917-A2945.

[50] Perrin G, Soize C, Duhamel D, and Funfschilling C (2013) Karhunen-Loève expansion revisited for vector-valued random fields: scaling, errors and optimal basis. J. Comput. Phys. 242(1): 607-622.

[51] Perrin G, Soize C, Duhamel D, and Funfschilling C (2014) A posterior error and optimal reduced basis for stochastic processes defined by a set of realizations. SIAM/ASA J. Uncertainty Quantification, 2: 745-762.

[52] Puig B, Poirion F, and Soize C (2002) Non-Gaussian simulation using Hermite polynomial expansion: Convergences and algorithms. Probab. Eng. Mech. 17(3): 253-264. 
[53] Rozanov YA (1998) Random Fields and StochasticPartial Differential Equations. Kluwer Academic Publishers, Dordrecht.

[54] Serfling RJ (1980) Approximation Theorems of Mathematical Statistics. John Wiley \& Sons, New York.

[55] Soize C (1994) The Fokker-Planck Equation for Stochastic Dynamical Systems and its Explicit Steady State Solutions. World Scientific Publishing Co Pte Ltd, Singapore.

[56] Soize C (2004) Random-field model for the elasticity tensor of anisotropic random media. CR Mecanique 332: 1007-1012.

[57] Soize C, Ghanem R (2004) Physical systems with random uncertainties : Chaos representation with arbitrary probability measure. SIAM J. Sci. Comput. 26(2): 395-410.

[58] Soize C (2006) Non Gaussian positive-definite matrix-valued random fields for elliptic stochastic partial differential operators. Comput. Methods Appl. Mech. Eng. 195(1-3): 26-64.

[59] Soize C (2008) Construction of probability distributions in high dimension using the maximum entropy principle. Applications to stochastic processes, random fields and random matrices. Int. J. Numer. Methods Eng. 76(10): $1583-1611$.

[60] Soize C (2008) Tensor-valued random fields for meso-scale stochastic model of anisotropic elastic microstructure and probabilistic analysis of representative volume element size. Probab. Eng. Mech. 23(2-3): 307-323.

[61] Soize C, Ghanem R (2009) Reduced chaos decomposition with random coefficients of vector-valued random variables and random fields. Comput. Methods Appl. Mech. Eng. 198(21-26): 1926-1934.

[62] Soize C (2010) Identification of high-dimension polynomial chaos expansions with random coefficients for non-Gaussian tensor-valued random fields using partial and limited experimental data. Comput. Methods Appl. Mech. Eng. 199(33-36): 2150-2164.

[63] Soize C, Desceliers C (2010) Computational aspects for constructing realizations of polynomial chaos in high dimension. SIAM J. Sci. Comput. 32(5): 2820-2831. 
[64] Soize C (2011) A computational inverse method for identification of nonGaussian random fields using the Bayesian approach in very high dimension. Comput. Methods Appl. Mech. Eng. 200(45-46): 3083-3099.

[65] Soize C (2012) Stochastic Models of Uncertainties in Computational Mechanics. American Society of Civil Engineers (ASCE), Reston.

[66] Soize C (2015) Polynomial chaos expansion of a multimodal random vector. SIAM/ASA J. Uncertainty Quantification 3(1): 34-60.

[67] Spall JC (2003) Introduction to Stochastic Search and Optimization. John Wiley \& Sons, Hoboken.

[68] Stuart AM (2010) Inverse problems: A bayesian perspective. Acta Numerica 19: 451-559.

[69] Ta QA, Clouteau D, and Cottereau R (2010) Modeling of random anisotropic elastic media and impact on wave propagation. Eur. J. Comput. Mech. 19(12-3): 241-253.

[70] Talay D (1995) Simulation and numerical analysis of stochastic differential systems. In: Kree P, Wedig W (eds) Probabilistic Methods in Applied Physics, Lecture Notes in Physics, 451, Springer-Verlag, Heidelberg, pp 5496.

[71] Talay D (2002) Stochastic Hamiltonian system: exponential convergence to the invariant measure and discretization by the implicit Euler scheme. Markov Processes and Related Fields 8: 163-198.

[72] Tarantola A (2005) Inverse problem Theory and Methods for Model Parameter Estimation. SIAM, Philadelphia.

[73] Tipireddy R, Ghanem R (2014) Basis adaptation in homogeneous chaos spaces. J. Comput. Physics 259: 304-317.

[74] Vanmarcke E (2010) Random Fields, Analysis and Synthesis (Revised and Expanded New Edition). World Scientific, Singapore.

[75] Walpole LJ (1981) Elastic behavior of composite materials: theoretical foundations. Adv. Appl. Mech. 21: 169-242. 
[76] Walter E, Pronzato L (1997) Identification of Parametric Models from Experimental Data. Springer-Verlag, Berlin.

[77] Wan XL, Karniadakis GE (2006) Multi-element generalized polynomial chaos for arbitrary probability measures. SIAM J. Sci. Comput. 28(3): 901928.

[78] Xiu DB, Karniadakis GE (2002) Wiener-Askey polynomial chaos for stochastic differential equations. SIAM J. Sci. Comput. 24(2): 619-644.

[79] Zienkiewicz OC and Taylor RL (2005) The Finite Element Method For Solid And Structural Mechanics, Sixth edition. Elsevier, Butterworth-Heinemann, Amsterdam. 